\title{
QUADRATIC SYSTEMS WITH A UNIQUE FINITE REST POINT
}

\author{
B. Coll, A. Gasull, J. Llibre
}

\begin{abstract}
We study phase portraits of quadratic systems with a unique finite singularity. We prove that there are 111 diferent phase portraits without limit cycles and that 13 of them are realizable with exactly one limit cycle. In order to finish completely our study two problems remain open: the realization of one topologically possible phase portrait, and to determine the exact number of limit cycles for a subclass of these systems.
\end{abstract}

\section{0 . Introduction and statement of the main results}

We consider the differential system $\dot{x}=d x / d t=P(x, y), \dot{y}=d y / d t=Q(x, y)$ where $P$ and $Q$ are polynomials of second degree with real constant coefficicnts, and $x, y, t$ are also real. We call such systems quadratic systems, $Q S$, for short. We assume that these systems have a unique finite singularity, and we denote them by QS1.

Our goal is to give all the possible phase portraits (modulus homeomorphisms and changes of the scale of the independent variable $t$ ) of the $Q S 1$ on the sphere of Poincaré (see [G] and [S]). Note that in this study we must take into account the number of limit cycles that the QS1 can have. This last problem is the most important difference between this classification and other similar works, see for instance [GLL], [Re].

We prove that there are 111 different phase portraits for $Q S 1$ without limit cycles. Furthermore 13 of them are also realizables by $Q S 1$ that have exactly one limit cycle; the phase portraits $e_{5}, e_{7}, e_{8}$ and $e_{9}$ are determinated modulo their number of limit cycles (see Figure 5.16) and we do not know if the phase portrait $e_{13}$ of the same figure exists for some QS1. More specifically, to finish completely our study, two problems have resisted our analysis:

(P1) Determine the maximum number of limit cycles that the $Q S 1 \dot{x}=$ $=y+p x^{2}+x y, \dot{y}=-x+b y+(\ell+b p) x^{2}+(m+b) x y$ has with conditions $p>0$, $(1+\ell)^{2}-4 p m<0$ and $(m+b-p)^{2}-4(\ell+b p)>0$.

(P2) Determine if there is some QS1 that has phase portrait $e_{13}$ of Figure 5.16 . 
We think that the answer for (P1) will be that the system considered has at most three limit cycles. About (P2), we think that phase portrait $e_{13}$ will not be realizable by $Q S 1$.

Now we shall give a scheme of the proof of the above results. First at all we need the following notation:

Let $X(x, y)$ be an analytic vector field, and suppose that the origin is an isolated singularity. Then, we say that $(0,0)$ is a singularity of type:

$e$ if the determinant of the linear part $D X(0,0)$ is not zero,

$s$ if the linear part $D X(0,0)$ has a unique eigenvalue equal to zero,

$h$ if the linear part $D X(0,0)$ has two eigenvalues equal to zero and it is not identically zero.

$t$ if the linear part is identically zero.

We shall use this notation for the finite singularity of the $Q S 1$, and the same notation, but with capital letters when we consider infinite singularities (i.e. singularities on the equator of the Poincare sphere). When the entire equator of the Poincare sphere is formed by critical points we shall say that the $Q S$ is degenerate.

Remember that the singularities of type $e$ or $s$ are called elementary singularities.

In Section 1 we give a classification of all the QS1 with a reduced number of parameters (see Lemma 1.2). We subdivide them according with the type of their infinite singularities. The results are given in Tables 1.1-1.6. After, we prove that several cases of these tables are topologically equivalent. In Table I.17 we summarize all the cases that we must to study. In Section I we give also the phase portraits for the homogeneous $Q S$ that have been studied in several previous papers (see Figure 1.1).

In Section 2 we give an affine classification of all the $Q S 1$ that are degenerate or linear. Furthermore, in Figure 2.1 we give all their phase portraits. Hence, in the following sections we do not consider $Q S 1$ degenerate or linear.

In Section 3 we study the phase portraits for $Q S 1$ that have a finite rest point of type $h$. We know that these systems have no limit cycles. By making a study case by case we obtain that their phase portraits are homeomorphic to one of the phase portraits of Figures 3.1 or 3.4 .

Section 4 deals with $Q S 1$ that have the finite rest point of type $e$ or $s$, but that have no limit cycles. The problem is more complicated that in the above sections because there are a lot of topological possibilities for their phase portraits, especially when the finite singularity is of saddle-node type. In order to study these systems we subdivide them into the following three cases:

(A) QS1 with some non-elementary singularity at infinity.

(B) QSI with three elementary singularities at infinity.

(C) QS1 with exactly two elementary singularities at infinity.

Case (A) is studied case by case. Note that when the singularity at infinity is non-elementary there are many topological possibilities. The results obtained 
are given in Figures 4.1 and 4.2 .

Cases (B) and (C) are studied in a similar way. First, we consider them with the finite rest point of index 1 or -1 , i.e. subcases (BI) and (C1). For these subcases is easy to do a topological classification of all their possible phase portraits. The results obtained are showed in Figures $4.3,4.4$ and 4.10 . The realizations of the pictures of these figures are shown in Tables 4.1, 5.3 and 5.4. Note that the phase portraits $E_{53}, E_{54}$ of Figure 4.10 , are equivalent to the phase portrait $e_{13}$ of Figure 5.16, i.e. is the one of problem (P2). When we consider cases (B) and (C) with the finite rest point of index 0 we obtain subcases (B2) and (C2). Subcase (B2) with the indices at infinity $(1,0,0)$ is studied case by case and the results obtained showed in Figure 4.5. The remainder subcases are subcase (B2) with singularities at infinity of indices $(1,1,-1)$ (denoted by B2.b) and (C2). We study them by considering all the topological possibilities (taking into account also some special properties of the $Q S$, for instance Proposition 3.2). The phase portraits obtained are showed in Figures 4.6 and 4.11 , and their realizations are given in Tables 4.2 and 4.3.

In Section 5 we study the $Q S 1$ that can have limit cycles. First, we write them in a more suitable form (see Proposition 5.1). In this new form, they are semicomplete families of rotated vector fields (SFR) with parameter $b$, and so we have more information about the evolution of their limit cycles when $b$ changes (see [Du], [PI]).

In the Appendix we enunciate some results of several papers about uniqueness of limit cycles of $Q S$. These results allow us to prove the uniqueness of the limit cycles for all systems of Proposition 5.1 except the system that we referred to problem (P1).

From the results of this appendix, the properties of the SFR and some particular propertics of our $Q S 1$, we obtain Figures $5.2,5.3,5.4,5.5,5.6,5.7,5.8$, $5.9,5.10$ and 5.14 that give us the phase portraits of systems (a), (b), (c) and (d) of Proposition 5.1. We obtain also some properties about system (e) of this proposition (see Figures 5.11, 5.12, 5.13, 5.15, 5.16 and 5.17).

In this paper we use also some results on weak focus (see [Che] or Appendix $B$ of [CGL]) and the standard results on the local behaviour of a vector field in a neighbourhood of a singularity (see for instance [ALGM]). We will refer in order to simplify the notation, to Theorems $E, S$ and $H$ when we use such theorems about singularities with this kind of degeneracy. Sometimes we are interested in knowing if an invariant straight line through a singular point is formed by separatrices. We solve this question by using the blow up method.

\section{Classification of the QS with a unique finite rest point}

In a first step we need a general classification of all the QS. In this way we have the following result. 
Lemma 1.1. (see Lemma 1 of [GLL]). The quadratic system

$$
\dot{x}=\frac{d x}{d t}=P(x, y), \quad \dot{y}=\frac{d y}{d t}=Q(x, y)
$$

is affine equivalent, scaling the variable $t$ if necessary, to one of the following ones:

$$
\begin{array}{lll}
(I) & \dot{x}=1+x y, & \dot{y}=Q(x, y) \\
(I I) & \dot{x}=x y, & \dot{y}=Q(x, y) \\
(I I I) & \dot{x}=y+x^{2}, & \dot{y}=Q(x, y) \\
(I V) & \dot{x}=y, & \dot{y}=Q(x, y) \\
(V) & \dot{x}=-1+x^{2}, & \dot{y}=Q(x, y) \\
(V I) & \dot{x}=1+x^{2}, & \dot{y}=Q(x, y) \\
(V I I) & \dot{x}=x^{2}, & \dot{y}=Q(x, y) \\
(V I I I) & \dot{x}=x, & \dot{y}=Q(x, y) \\
(I X) & \dot{x}=1, & \dot{y}=Q(x, y) \\
(X) & \dot{x}=0, & \dot{y}=Q(x, y)
\end{array}
$$

where $Q(x, y)=d+a x+b y+\ell x^{2}+m x y+n y^{2}$.

We shall say that an isolated singularity $\left(x_{0}, y_{0}\right)$ of a vector field $X$ on the plane is a singularity of type:

$e$ if $\left(x_{0}, y_{0}\right)$ is a nondegenerate singularity;

$s$ if the linear part of $D X\left(x_{0}, y_{0}\right)$ has a unique eigenvalue equal to zero;

$h$ if the linear part of $D X\left(x_{0}, y_{0}\right)$ has the two eigenvalues equal to zero, and $D X\left(x_{0}, y_{0}\right) \neq 0$.

$t$ if $D X\left(x_{0}, y_{0}\right) \equiv 0$.

The singularities of type $e$ or $s$ are called elementary singularities.

We shall denote by (A.a) a $Q S 1$ of type (A) whose finite singularity is of type $a$, where $A \in\{(I),(I I), \ldots,(X)\}$ and $a \in\{e, s, h, t\}$. By using this notation we can give a classification of the $Q S 1$.

Lemma 1.2. A QS1 is affine equivalent, scaling the variable $t$ if necessary to one of the following cases:

(a) (I.e) $\dot{x}=y-x^{2}+x y, \dot{y}=a x+b y+Q_{2}(x, y)$ with $a \neq 0$;

(I.s) $\dot{x}=y-x^{2}+x y, \dot{y}=b y+Q_{2}(x, y)$ with $b \neq 0$;

(I.h) $\dot{x}=y-x^{2}+x y, \dot{y}=Q_{2}(x, y)$.

(b) $(I I . e),(I I . s)$ or $(I I . h)$.

(c) $(I M I . e) \quad \dot{x}=y+x^{2}, \dot{y}= \pm x+b y+Q_{2}(x, y)$ with $n=0$ and, either $m \neq 0$ and $(\ell-b)^{2} \pm 4 m<0$, or $m=0$ and $\ell=b$;

(III.s) $\dot{x}=y+x^{2}, \dot{y}=y+Q_{2}(x, y)$ with either $n \neq 0$ and $m^{2}-4 n(\ell-1)<0$, or $n \neq 0, m=0$ and $\ell=1$, or $n=0, m \neq 0$ and $\ell=1$, or $n=m=0$ and $\ell \neq 1$;

$(I I I, h) \quad \dot{x}=y+x^{2}, \dot{y}=Q_{2}(x, y)$ with either $n \neq 0$ and $m^{2}-4 n \ell<0$, or $n \neq 0, m=\ell=0$, or $n=\ell=0, m \neq 0$, or $n=m=0, \ell \neq 0$. 
(d) (IV.e) $\dot{x}=y, \dot{y}= \pm x+b y+Q_{2}(x, y)$ with $b \geq 0$ and $\ell=0$;

(IV.s) $\dot{x}=y, \dot{y}=y+Q_{2}(x, y)$ with $\ell \neq 0$;

(IV.h) $\dot{x}=y, \dot{y}=Q_{2}(x, y)$ with $\ell \neq 0$.

(e) (V.e) $\dot{x}=x^{2}-1, \dot{y}=d+b y+\ell x^{2}+m x y$ with $m \neq 0$ and $d+\ell \neq 0$;

(V.s) $\dot{x}=x^{2}-1, \dot{y}=d+a x+b y+\ell x^{2}+m x y+y^{2}$ with $(b+m)^{2}-$ $-4(d+a+\ell)=0$ and $(b-m)^{2}-4(d-a+\ell)<0$.

Furthermore, we can assume that the singularity of system (V) is on the straight line $x=1$.

(f) (VII.s) $\dot{x}=x^{2}, \dot{y}=y+Q_{2}(x, y)$ with $n=0$;

$(V I I, h) \quad \dot{x}=x^{2}, \dot{y}=x+Q_{2}(x, y)$ with $n=1$.

(g) We split system (VIII.e) into two subsystems:

(VIII.e $\left.e_{1}\right) \quad \dot{x}=x, \dot{y}=b y+Q_{2}(x, y)$ with $b \neq 0$ and $n=0$;

$\left(V I I I e_{2}\right) \quad \dot{x}=x, \dot{y}=x+y+Q_{2}(x, y)$ with $n=0$;

(VIII.s) $\quad \dot{x}=x, \dot{y}=Q_{2}(x, y)$ with $n \neq 0$.

(h) A homogenous quadratic system (i.e. $\dot{x}=P_{2}(x, y), \dot{y}=Q_{2}(x, y)$ ). Here $Q_{2}(x, y)=\ell x^{2}+m x y+n y^{2}, P_{2}(x, y)=L x^{2}+M x y+N y^{2}$.

Proof: By Lemma 1.1 we can consider that the $Q S 1$ is of one of the types, from (I) to (X). Clearly systems (VI) and (IX) have no finite singularities. If some system has a singularity of type $t$ we can translate it to $(0,0)$ and so we obtain $(h)$. So systems $(\mathrm{X})$ are contained in $(h)$.

In the case (I), let $\left(x_{0}, y_{0}\right)$ be the critical point of the system. Since $x_{0} \neq 0$, in the coordinates $x_{1}=-x_{0}\left(x-x_{0}\right), y_{1}=-x_{0} x+y / x_{0}+x_{0}^{2}+1 / x_{0}^{2}, t_{1}=x_{0} t$ system (I) can be written as $\dot{x}_{1}=y_{1}-x_{1}^{2}+x_{1} y_{1}, \dot{y}_{1}=Q^{\prime}\left(x_{1}, y_{1}\right)$. So (a) follows.

In the coordinates $x_{1}=x-x_{0}, y_{1}=2 x_{0} x+y-x_{0}^{2}$, where $\left(x_{0}, y_{0}\right)$ is the critical point of system (III), it can be written as $\dot{x}=y+x^{2}, \dot{y}=a^{\prime} x+b^{\prime} y+$ $+\ell^{\prime} x^{2}+m^{\prime} x y+n^{\prime} y^{2}$, where we always omit the subscript one and $a^{\prime}=a+2(\ell-$ $-b) x_{0}-3 m x_{0}^{2}+4 n x_{0}^{3}$. If $a^{t} \neq 0$, then system (III) can be written in the form (III.e) by using the change of variables $x_{1}=\left|a^{\prime}\right|^{-1 / 2} x, y_{1}=\left|a^{\prime}\right|^{-1} y, t_{1}=$ $\left|a^{t}\right|^{1 / 2} t$. If $a^{\prime}=0$ and $b^{\prime} \neq 0$ then the transformation $x_{1}=\left(b^{\prime}\right)^{-1} x, y_{1}=\left(b^{\prime}\right)^{-2} y$, $t_{1}=b^{\prime} t$ writes system (III) in the form (III.s). Lastly, if $a^{\prime}=b^{\prime}=0$ then we have system (III.h), and (c) holds.

Without loos of generality we can assume that the singularity is at the origin for systems (IV), (VII) and (VIII).

Now, for (IV) we have $d=0$. If $b=0$ then (IV) can be written as (IV.h) or (IV.e) according to $a$ is zero or nonzero. If $b \neq 0$ and $a \neq 0$ using the change of variables $x_{1}=b|b|^{-1}|a|^{1 / 2} x, y_{1}=y, t_{1}=b|b|^{-1}|a|^{1 / 2} t$ system (IV) becomes in system (IV.e). If $b \neq 0$ and $a=0$ then the transformation $x_{1}=b x, y_{1}=y$, $t_{1}=b t$ changes (IV) into the form (IV.s). In short (d) follows.

By using the change of variables $x_{1}=-x, y_{1}=y, t_{1}=-t$ (if necessary) we can assume that the singularity of (V) is on the straight line $x=1$. Therefore, if the singularity of (V) is of type $e$ then $n=0$ and $m=b \neq 0$. Now transformation $x_{1}=x, y_{1}=y+a / m$ changes system $(\mathrm{V}, \mathrm{e})$ into the form given in (e). 
If $n \neq 0$ then the change of variables $x_{1}=x, y_{1}=n y, t_{1}=t$ changes system (V) into the form (V.s) of (e).

Making a linear transformation which changes the linear part of systems (VII) and (VIII) into the canonical Jordan form and scaling the variables (if necessary), we obtain (f) and (g).

Now we shall subdivide the cases given in Lemma 1.2, except case (h), ac cording to the kind of singularities that the $Q S$ has on the equator of Poincarc sphere.

For the infinite singularities, we will follow the same notation that for the finite singularity but with capital letters. If a $Q S$ is such that all the points on the equator of the Poincare sphere are singularities we shall say that it is degenerate. The results of all these subdivisions are given in Tables $1.1-1.16$ below.

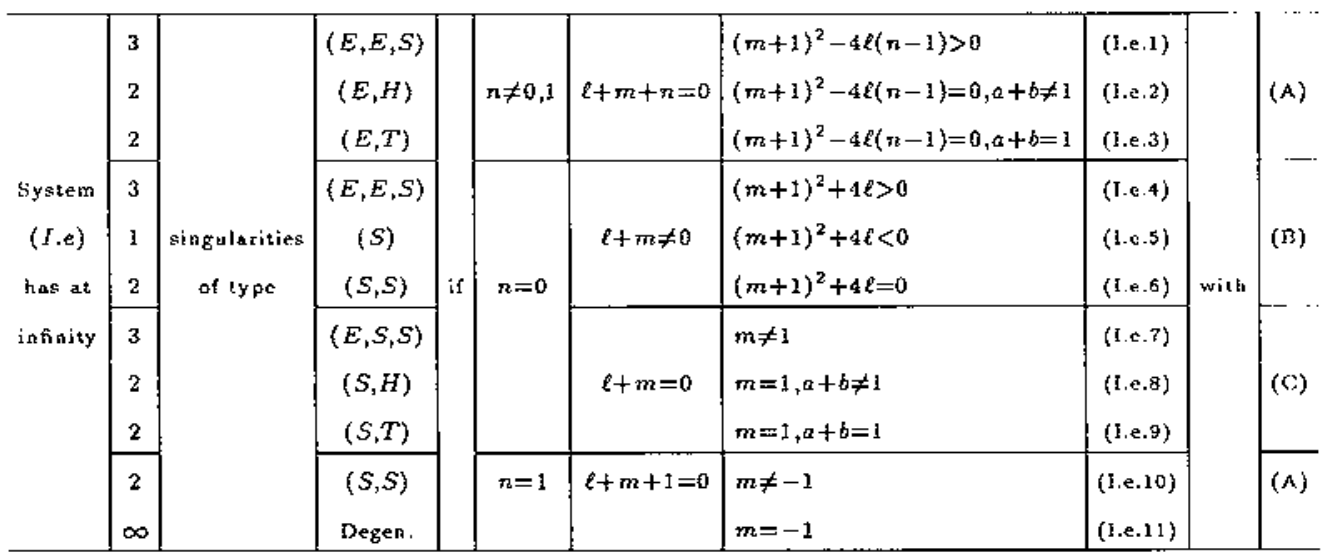

Table 1.1. The singularities at infinity for system (I.e), where

(A) $a \neq 0$, and $(2 a+b+\ell)^{2}-4 a(a+b+2 \ell+m)<0$, or $a+b+2 \ell+m=$ $=2 a+b+\ell=0$.

(B) $a \neq 0$, and $(a+b+\ell)^{2}-4 a(\ell+m)<0$, or $b=m, a=\ell+m<0$.

(C) $a \neq 0$, and $b+\ell=0$. 


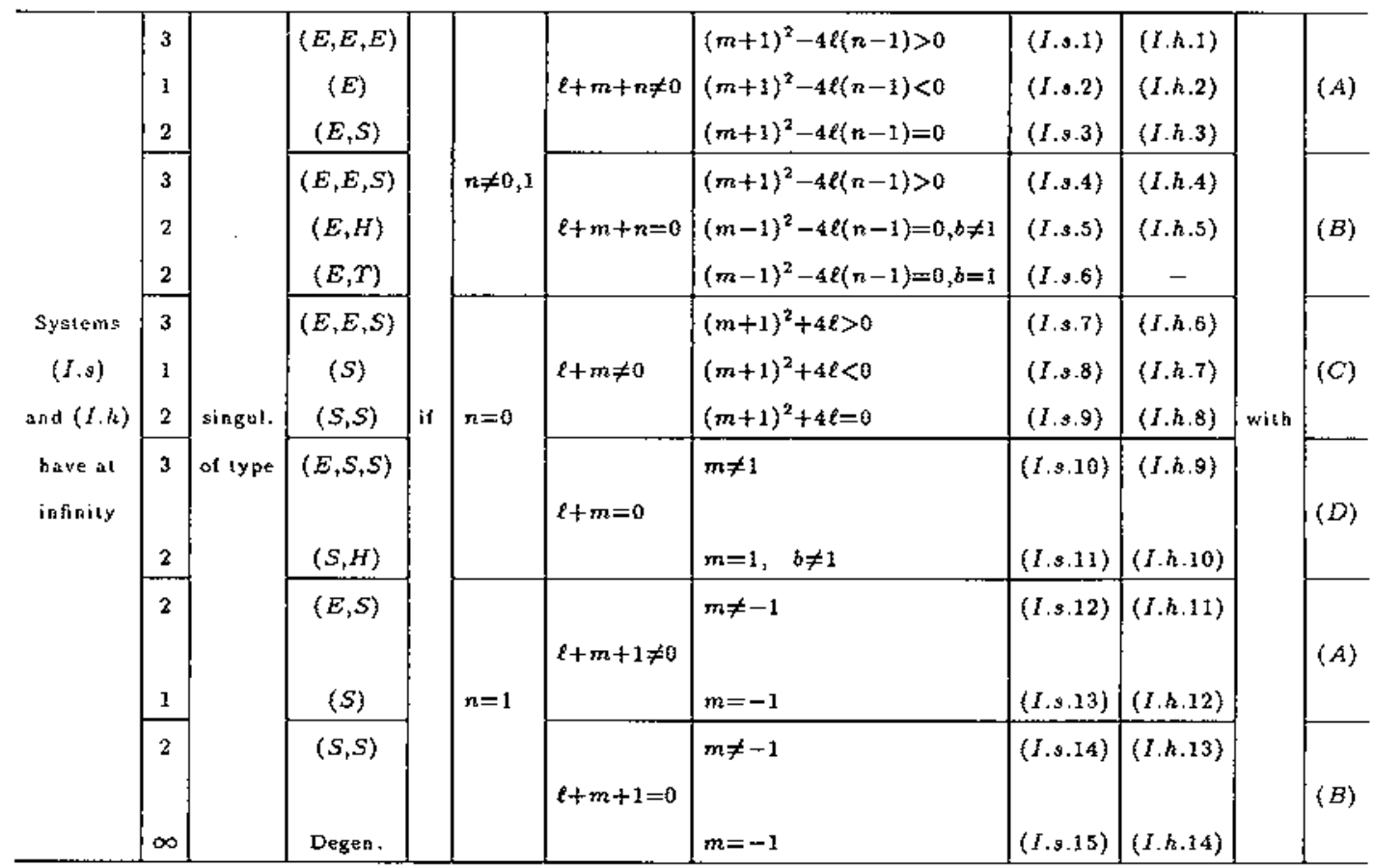

Table 1.2. The singularities at infinity for systems (I.s) and (I.h), where

(A) $a=0$, and $(b+2 \ell+m)^{2}-4(b+\ell)(\ell+m+n)<0$ or $b+\ell=\ell+m=0$.

(B) $a=0$, and $b+\ell=0$ or $b+2 \ell+m=0$.

(C) $a=0$, and $b-m=0$ or $b+\ell=0$.

(D) $a=0$, and $b+\ell \neq 0$.

Furthermore, $b \neq 0$ in case (I.s) and $b=0$ in case (I.h).

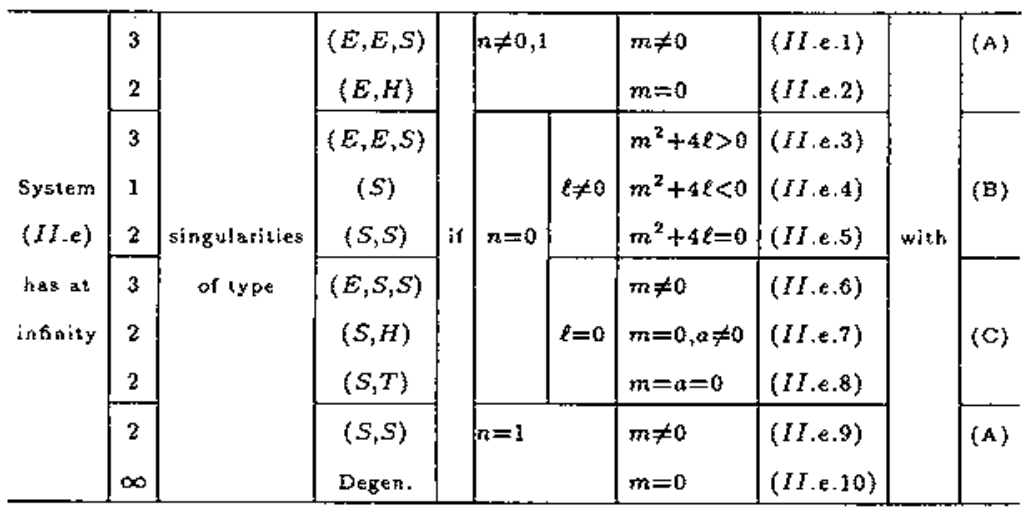

Table 1.3. The singularities at infinity for system (II.e), where 

(A) $b^{2}-4 n d<0, \ell=0, a \neq 0$.
(B) $a^{2}-4 \ell d<0, b \neq 0$.
(C) Either $a=0, b \neq 0, d \neq 0$, or $a \neq 0, b=0, d \neq 0$.

\begin{tabular}{|c|c|c|c|c|c|c|c|c|c|}
\hline & $\begin{array}{l}3 \\
1 \\
2\end{array}$ & & $\begin{array}{c}(E, E, E) \\
(E) \\
(E, S)\end{array}$ & $n \neq 0,1$ & $\ell \neq 0$ & $\begin{array}{l}m^{2}-4(n-1) \ell>0 \\
m^{2}-4(n-1) \ell<0 \\
m^{2}-4(n-1) \ell=0\end{array}$ & $\begin{array}{l}(I I . s .1) \\
(I I . s .2) \\
(I I . s .3)\end{array}$ & & $(A)$ or $(B)$ \\
\hline & 2 & & $\begin{array}{c}(E, E, S) \\
(E, T)\end{array}$ & & $\hat{\ell}=0$ & $\begin{array}{l}m \neq 0 \\
m=0\end{array}$ & $\begin{array}{l}(I I . s .4) \\
(I I .3 .5)\end{array}$ & & (C) \\
\hline $\begin{array}{l}\text { System } \\
(1 I . s) \\
\text { has \&l }\end{array}$ & $\begin{array}{l}3 \\
3 \\
2 \\
\end{array}$ & $\begin{array}{l}\text { singularities } \\
\text { of type }\end{array}$ & $\begin{array}{c}(E, E, S) \\
(S) \\
(S, S)\end{array}$ & $n=0$ & $\ell \neq 0$ & $\begin{array}{l}m^{2}+4 \ell>0 \\
m^{2}+4 \ell<0 \\
m^{2}+4 \ell=0\end{array}$ & $\begin{array}{l}(I . s .6) \\
(H . s .7) \\
(I I .4 .8)\end{array}$ & with & $(D)$ or $(E)$ \\
\hline infinity & 3 & & $\begin{array}{l}(E, S, S) \\
(S, H)\end{array}$ & & $\ell=0$ & $\begin{array}{l}m \neq 0 \\
m=0\end{array}$ & $\begin{array}{l}(11 . s .9) \\
(11 . s .10) \\
\end{array}$ & & $(F)$ \\
\hline & \pm & & $\begin{array}{l}(E, S) \\
(S)\end{array}$ & & $\ell \neq 0$ & $\begin{array}{l}m \neq 0 \\
m=0\end{array}$ & $\begin{array}{l}\text { (II.s.11) } \\
(I I . s .12)\end{array}$ & & $(A)$ or $(B)$ \\
\hline & 2 & & $\begin{array}{l}(S, S) \\
\text { Degen. }\end{array}$ & $n=1$ & $\ell=0$ & $\begin{array}{l}m \neq 0 \\
m=0\end{array}$ & $\begin{array}{l}(\text { (II.s.13) } \\
\text { (II.s.14) }\end{array}$ & & (C) \\
\hline
\end{tabular}

Table 1.4. The singularities at infinity for system (II.s), where
(A) $n \neq 0, \ell \neq 0, b^{2}-4 n d=0, a^{2}-4 \ell d<0$.
(B) $n \neq 0, \ell \neq 0, b^{2}-4 n d<0, a^{2}-4 \ell d=0,2 b \ell-m a \neq 0$.
(C) $n \neq 0, \ell=0, b^{2}-4 n d=0, a=0, d \neq 0$.
(D) $n=0, \ell \neq 0, b=0, d \neq 0, a^{2}-4 \ell d=0, m \neq 0$.
(E) $n=0, \ell \neq 0, d=0, b \neq 0, a=0$.
(F) $n=0, \ell=0, d=0, b \neq 0, a \neq 0$. 


\begin{tabular}{|c|c|c|c|c|c|c|c|c|c|c|}
\hline & $\begin{array}{l}3 \\
1 \\
2\end{array}$ & & $\begin{array}{l}(E, E, E) \\
(E) \\
(E, S)\end{array}$ & & $n \neq 0,1$ & $\ell \neq 0$ & $\begin{array}{l}m^{2}-4(n-1) \ell>0 \\
m^{2}-4(n-1) \ell<0 \\
m^{2}-4(n-1) \varepsilon=0\end{array}$ & $\begin{array}{l}(I I h .1) \\
(I I . h .2) \\
(I I h .3)\end{array}$ & & $(A)$ \\
\hline System & 3 & & $\begin{array}{l}(E, E, S) \\
(E, H)\end{array}$ & & & $\ell=0$ & $\begin{array}{l}m \neq 0 \\
m=0\end{array}$ & $\begin{array}{l}(I I . h .4) \\
(I I, h .5)\end{array}$ & & $(B)$ \\
\hline $\begin{array}{l}(1 f . k) \\
\text { bas at }\end{array}$ & 3 & $\begin{array}{l}\text { singularities } \\
\text { of type }\end{array}$ & $\begin{array}{l}(E, E, S) \\
(S)\end{array}$ & $\pi$ & $n=0$ & $\ell \neq 0$ & $\begin{array}{l}\ell>0 \\
\ell<0\end{array}$ & $\begin{array}{l}(I I, h .6) \\
(I I h .7)\end{array}$ & with & (C) \\
\hline infinity & 2 & & $\begin{array}{l}(E, S) \\
(S)\end{array}$ & & & $\ell \neq 0$ & $\begin{array}{l}m \neq 0 \\
m=0\end{array}$ & $\begin{array}{l}(I I, h .8) \\
(I I, h .9)\end{array}$ & & $(A)$ \\
\hline & 2 & & $\begin{array}{l}(S, S) \\
\text { Degen. }\end{array}$ & & $n=1$ & $\ell=0$ & $\begin{array}{l}m \neq 0 \\
m=0\end{array}$ & $\begin{array}{l}(I . h .10) \\
(I I, .11)\end{array}$ & & (B) \\
\hline
\end{tabular}

Table 1.5. The singularities at infinity for system (II.h), where

(A) $n \neq 0, \ell \neq 0, b^{2}-4 n d<0, a^{2}-4 \ell d=0,2 b \ell-m a=0$.

(B) $n \neq 0, \ell=0, b=0, a \neq 0, d=0$.

(C) $n=0, \ell \neq 0, b=0, d \neq 0, a^{2}-4 \ell d=0, m=0$.

\begin{tabular}{|c|c|c|c|c|c|c|}
\hline System & 2 & singularities & $(E, H)$ & & $m \neq 1$ & (III.e.1) \\
\hline$(I I I . e)$ has & 1 & of eype & (H) & if & $m=1, \varepsilon \neq 0$ & (III.e.2) \\
\hline at infinity & $\infty$ & & Degen. & & $m=1, \ell=0$ & $(I M, e, 3)$ \\
\hline
\end{tabular}

Table 1.6. The singularities at infinity fo: system (III.e).

\begin{tabular}{|c|c|c|c|c|c|c|c|c|}
\hline $\begin{array}{l}\text { Systems } \\
(I I I . s) \text { and }\end{array}$ & $\begin{array}{l}3 \\
1 \\
2\end{array}$ & singularities & $\begin{array}{c}(E, E, E) \\
(E) \\
(E, S)\end{array}$ & if & $n \neq 0$ & $\begin{array}{l}(m-1)^{2}-4 n \ell>0 \\
(m-1)^{2}-4 n \ell<0 \\
(m-1)^{2}-4 n \ell=0\end{array}$ & $\begin{array}{l}(I I I .3 .1) \\
(I I I .9 .2) \\
(I I I . s .3)\end{array}$ & $\begin{array}{l}(I I I . k .1) \\
(I I . h .2) \\
(I I I h .3)\end{array}$ \\
\hline$(I I I . h)$ & 2 & & $(E, H)$ & & & $m \neq 1$ & $(I I I . s .4)$ & $(I I I . h .4)$ \\
\hline have at & 1 & & $(H)$ & & $n=0$ & $m=1, \ell \neq 0$ & (III.s.5) & - \\
\hline infarity & $\infty$ & & Degen. & & & $m=1, \ell=0$ & - & $(M I M .5)$ \\
\hline
\end{tabular}

Table 1.7. The singularities at infinity for systems (III.s) and (III.h). 


\begin{tabular}{|c|c|c|c|c|c|c|c|}
\hline \multirow{2}{*}{$\begin{array}{l}\text { System } \\
\text { (IV.e) has } \\
\text { at infinity }\end{array}$} & \multirow{2}{*}{$\begin{array}{l}3 \\
2 \\
2\end{array}$} & \multirow[t]{2}{*}{$\begin{array}{c}\text { singularities } \\
\text { of type }\end{array}$} & \multirow{2}{*}{$\begin{array}{c}(E, S, S) \\
(E, H) \\
(S, H)\end{array}$} & \multirow[t]{2}{*}{ if } & \multirow{2}{*}{$\begin{array}{l}n \neq 0 \\
n=0\end{array}$} & \multirow{2}{*}{$\begin{array}{l}m \neq 0 \\
m=0 \\
m \neq 0\end{array}$} & \multirow{2}{*}{$\begin{array}{l}(I \text { V.e.1 }) \\
(I \text { V.e. } 2) \\
(I \text { V.e. } 3)\end{array}$} \\
\hline & & & & & & & \\
\hline System & $(I V$ & is & linear & when & & $=m=0$ & (IV.e.4) \\
\hline
\end{tabular}

Table 1.8. The singularities at infinity for system (IV.e).

\begin{tabular}{|c|c|c|c|c|c|c|c|}
\hline $\begin{array}{l}\text { System } \\
(I V . s) \\
\text { has al }\end{array}$ & $\begin{array}{l}3 \\
1 \\
2 \\
2\end{array}$ & $\begin{array}{c}\text { singularities } \\
\text { of type }\end{array}$ & $\begin{array}{c}(E, S, S) \\
(E) \\
(E, H) \\
\left(E, T^{*}\right)\end{array}$ & if & $n \neq 0$ & $\begin{array}{l}m^{2}-4 n \ell>0 \\
m^{2}-4 n \ell<0 \\
m^{2}-4 n \ell=0,2 n+m \neq 0 \\
m^{2}-4 n \ell=2 n+m=0\end{array}$ & $\begin{array}{l}(I V . s .1) \\
(I V . s .2) \\
(I V . s .3) \\
(I V . s .4)\end{array}$ \\
\hline infinity & $\begin{array}{l}2 \\
1\end{array}$ & & $\begin{array}{c}(S, H) \\
(H)\end{array}$ & & $n=\hat{0}$ & $\begin{array}{l}m \neq 0 \\
m=0\end{array}$ & $\begin{array}{l}(I V .8 .5) \\
(I V .8 .6)\end{array}$ \\
\hline
\end{tabular}

Table 1.9. The singularities at infinity for system (IV.s).

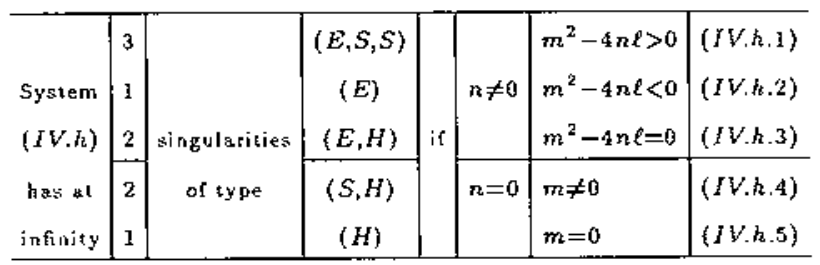

Table 1.10. The singularities at infinity for system (IV.h).

\begin{tabular}{c|c|c|c|c|c|c}
\hline Systern & 2 & singularities & $(E, T)$ & & $m \neq 1$ & $(V . e .1)$ \\
$(V . e)$ has at & 1 & of type & $(T)$ & if & $m=1, \ell \neq 0$ & $($ V.e.2) \\
infinity & $\infty$ & & Degea. & & $m=1, \ell=0$ & $($ V.e.3) \\
\hline
\end{tabular}

Table 1.11. The singularities at infinity for system (V.e).

\begin{tabular}{c|c|c|c|c|c|c}
\hline System & 3 & singularities & $(E, E, E)$ & & $(m-1)^{2}-4 \ell>0$ & $(V . s .1)$ \\
$(V, g)$ has at & 1 & of lype & $(E)$ & if & $(m-1)^{2}-4 \ell<0$ & $(V . s .2)$ \\
infinity & 2 & & $(E, S)$ & $(m-1)^{2}-4 \ell=0$ & $(V, s .3)$ \\
\hline
\end{tabular}

Table 1.12. The singularities at infinity for system (V.s). 


\begin{tabular}{c|c|c|c|c|l|l}
\hline System & 2 & singularities & $(E, T)$ & & $m \neq 1$ & $(V I I . s .1)$ \\
(VII.s).has & 1 & of type & $(T)$ & if & $m=1, \ell \neq 0$ & $(V I I . s .2)$ \\
at infinity & $\infty$ & & Degen. & & $m=1, \ell=0$ & $(V I I . s .3)$ \\
\hline
\end{tabular}

Table 1.13. The singularities at infinity for system (VII.s).

\begin{tabular}{c|c|c|c|c|c|c}
\hline System & 3 & singularities & $(E, E, E)$ & & $(m-1)^{2}-4 n \ell>0$ & $(V I I . h .1)$ \\
$(V I I . h)$ has & 1 & of lype & $(E)$ & if & $(m-1)^{2}-4 n \ell<0$ & $(V I I . h .2)$ \\
at infinity & 2 & & $(E, S)$ & $(m-1)^{2}-4 n \ell=0$ & $(V I I h .3)$ \\
\hline
\end{tabular}

Table 1.14. The singularities at infinity for systems (VII.h).

\begin{tabular}{|c|c|c|c|c|c|c|c|}
\hline $\begin{array}{c}\text { Systems (VIII,e }) \\
\text { and }\left(V I I, e_{2}\right) \text { have } \\
\text { at infuity }\end{array}$ & 1 & ingu & $(T)$ & & $m=0, l \neq 0$ & i) & $V$ \\
\hline & & & & & & & 3) \\
\hline
\end{tabular}

Table 1.15. The singularities at infinity for systems $\left(V I I I . e_{1}\right)$ and $\left(V I I I . e_{2}\right)$.

\begin{tabular}{c|c|c|l|l|l|l}
\hline System (VIIJ.s) & $\begin{array}{l}3 \\
1\end{array}$ & singularities & $(E, S, S)$ & $(E)$ \\
2 & of type & $(E, H)$ \\
has at infinity & & if & $m^{2}-4 n \ell>0$ & $($ VIII.s.1) \\
$m^{2}-4 n \ell<0$ & $(V I I I . s .2)$ \\
$m^{2}-4 n \ell=0, \ell \neq 0$ & $(V I I I . s .3)$ \\
\hline
\end{tabular}

Table 1.16. The singularities at infinity for system (VIII.s).

In this final classification there are some cases which are affine equivalent. Now we shall study these cases.

Lemma 1.3. (see Lemma 9 of $[$ GLL]). For $n \neq 0$ the system

$$
\dot{x}=P(x, y), \quad \dot{y}=d+a x+b y+n y^{2}
$$

with $a=0$ can be writien as

$$
\begin{array}{ll}
\dot{x}=x^{2}+1, & \dot{y}=Q(x, y) \\
\dot{x}=x^{2}, & \dot{y}=Q(x, y) \\
\dot{x}=x^{2}-1, & \dot{y}=Q(x, y)
\end{array}
$$


according to whether $k=b^{2}-4 n d$ is negative, zero or positive. If $a \neq 0$ then it can be written as

$$
\dot{x}=y+x^{2}, \quad \dot{y}=Q(x, y) .
$$

Remark 1.4. From this Lemma we need not to study cases (II.e.2), (II.e.10), (II.s.5), (II.s.14), (II.h.5), (II.h.11) and (IV.e.2).

Lemma 1.5. If system (I) (in the form given by Lemma 1.2) satisfies $\ell+$ $+m+n=0$ then by a linear change of coordinates plus a scaling of the variable $t$, it can be reduced either to (I) but fulfilling $\ell+m+n \neq 0$, or to another case of the ones given by Lemma 1.2.

Proof: Assume $n \neq 0$ and $\ell+m+n=0$, then the change of variables $x_{1}=-x+y, y_{1}=x$ changes our system to the form $\dot{x}=(-1+a+b) y+(b--$ $-1) x+x((m+2 n-1) y+n x), \dot{y}=x+y+x y$. If $-1+a+b=0$ and $m+2 n-1 \neq 0$ the new change of variables $x_{1}=x, y_{1}=(b-1)+(m+2 n-1) y+n x$ transforms it into a system of type (II). If $-1+a+b=0$ and $m+2 n-1=0$ we have system $\dot{x}=(b-1) x+n x^{2}, \dot{y}=x+y+x y$ hence, from Lemma 1.3 (after interchanging $x$ and $y$ ) we can reduce it to one of the types (V) or (VII). If $-1+a+b \neq 0$ the change of variables $x_{1}=x, y_{1}=(-1+a+b) y+(b-1) x$ write it like $\dot{x}=y+C x^{2}+D x y, \dot{y}=(-1+a+b) x+y+x y-(b-1) x^{2}$ whore $C=n-(b-1)(m+2 n-1) /(-1+a+b), D=(m+2 n-1) /(-1+a+b)$ and wc omit the subindex 1 . If $D=0$ and $C \neq 0$ the new variables $x_{1}=C x, y_{1}=C y$ transforms it into a system of type (III), and if $D=0$ and $C=0$ we have a system of type (IV). The case in which $D \neq 0$ and $C=0$ in the variablcs $x_{1}=1+D x$ and $y_{1}=D y$ it is of type (II), and the case in which $D \neq 0$ and $C \neq 0$ the change $x_{1}=D x, y_{1}=-D^{2} y / C, t_{1}=-C t / D$ converts it into a system of type (I) but with $n=0$.

Assume $n=0$ and $\ell+m=0$. The system becomes $\dot{x}=y-m x+x(-x+$ $+y-m x), \dot{y}=\left(b m-a-m^{2}\right) x+(m-b) y$ in the variables $x_{1}=m x-y, x_{1}=x$, after omiting the subindex 1 . If $b m-a-m^{2} \neq 0$ the change of variables $y_{1}=\left(b m-a-m^{2}\right) x+(m-b) y, x_{1}=y$ converts it into a system of type (IV). If $b m-a-m^{2}=0$, then $m-b \neq 0$ and with the change of variables $x_{1}=y$, $y_{1}=x, t_{1}=(m-b) t$ it becomes a system of type (VIII).

Remark 1.6. From this Lemmas we obtain that the cases from (I.e.1) to (I.e.3), from (I.e.7) to (I.e:11), (I.s.4), (I.s.5), (I.s.6), (I.s.10), (I.s.11), (I.s.14), (I.s.15), (I.h.4), (I.h.5), (I.h.9), (I.h.10), (I.h.13) and (I.h.14), are affine equivalent to other cases of Lermma 1.2 .

Lemma 1.7. (a) Systems $\dot{x}=x, \dot{y}=Q(x, y)$ and $\dot{x}=y, \dot{y}=Q(x, y)$ with $n \neq 0$ and $m^{2}-4 n \ell=0$ are affine equivalent to some systems contained in types (III), (V) and (VII).

(b) System $\dot{x}=x y, \dot{y}=d+a x+b y+m x y$ can be transformed by a linear change of coordinates into a system of one of the types (IV), (VIII) or (X). 
Proof: In the new variables $x_{1}=x, y_{1}=y+m x / 2 n$ the differential systems write $\dot{x}=P(x, y-m x / 2 n), \dot{y}=d+(2 a n-m b) x / 2 n+b y+n y^{2}+m P(x, y-$ $-m x / 2 n) / 2 n$, where $P(x, y)$ is either $x$ or $y$, and we omit the subindex 1 . Hence, from Lemma 1.3, (a) follows.

In the variables $x_{1}=x, y_{1}=y-m x$ the system of differential equations becomes $\dot{x}=x(y+m x), \dot{y}=d+(a+m) x+b y$ after omitting the subindex 1. This system can be transformed into a system of type (IV), (VIII) or (X) according with $a+m \neq 0 ; a+m=0, b \neq 0$ and $a+m=b=0$ (in this case $d$ must be zero), respectively. So, (b) is proved.

Remark 1.8. From this Lemma we do not need to study the following cases: (II.e.6), (II.e.7), (II.e.8), (II.s.9), (II.s.10), (IV.s.3), (IV.s.4), (VIII.s.3), (VIII.s.4) and (IV.h.3).

So, from Remarks $1.4,1.6$ and 1.8 the cases that we must study are summarized in Table 1.17 .

(1) Degenerate or linear systems:

Degenerate: (III.e.3), (III.h.5), (V.e.3), (VII.s.3).

Linear: (IV.e.4), (VIII. $\left.e_{1} .3\right)$, (VIII. $e_{2} .3$ ).

(2) Neither degenerate nor linear systems:

a) with a critical point of type $e$.

(I.e.4), (I.e.5), (I.e.6); (II.e.1), (II.e.3), (II.e.4), (II.e.5), (II.e.9); (III.e.1), (III.e.2); (IV.e.1), (IV.e.3); (V.e.1), (V.e.2); (VIIL.e.1. (VIII. $e_{2} .1$ ), (VIII. $\left.e_{1} .2\right)$, (VIII. $e_{2} .2$ ).

b) with a critical point of type $s$.

(I.s.1), (I.s.2), (I.s.3), (I.s.7), (I.s.8), (I.s.9), (I.s.12), (I.s.13); (II.s.1), (II.s.2), (II.s.3), (II.s.4), (II.s.6), (II.s.7), (II.s.8), (II.s.11), (II.s.12), (II.s.13); (III.s.I), (III.2.2), (III.s.3), (III.s.4), (III.s.5); (IV.s.1), (IV.s.2), (IV.s.5), (IV.s.6), (V.s.1), (V.s.2), (V.s.3); (VII.s.1), (VII.s.2); (VIII.s.1), (VIII.s.2).

c) with a critical point of type $h$.

(I.h.1), (I.h.2), (I.h.3), (I.h.6), (I.h.7), (I.h.8),(I.h.11), (I.h.12); (II.h.1), (II.h.2), (II.h.3), (II.h.4), (II.h.6), (II.h.7), (II.h.8), (II.h.9), (II.h.10); (III.h.1), (III.h.2), (III.h.3), (III.h.4); (IV.h.1), (IV.h.2), (IV.h.4), (IV.h.5); (VII.h.1), (VII.h.2), (VII.h.3).

d) with a critical point of type $t$, (i.e. homogeneous quadratic systems).

Table 1.17. Classification of all the $Q S 1$.

The phase portrait of homogenous polynomial vector fields on the plane, or on the Poincare sphere has been studied in many papers, see for instance [M], $[A]$ and [D]. In Figure 1.1 we give all the phase portraits for planar quadratic 
homogenous vector fields on the Poincaré sphere with only one finite critical point.
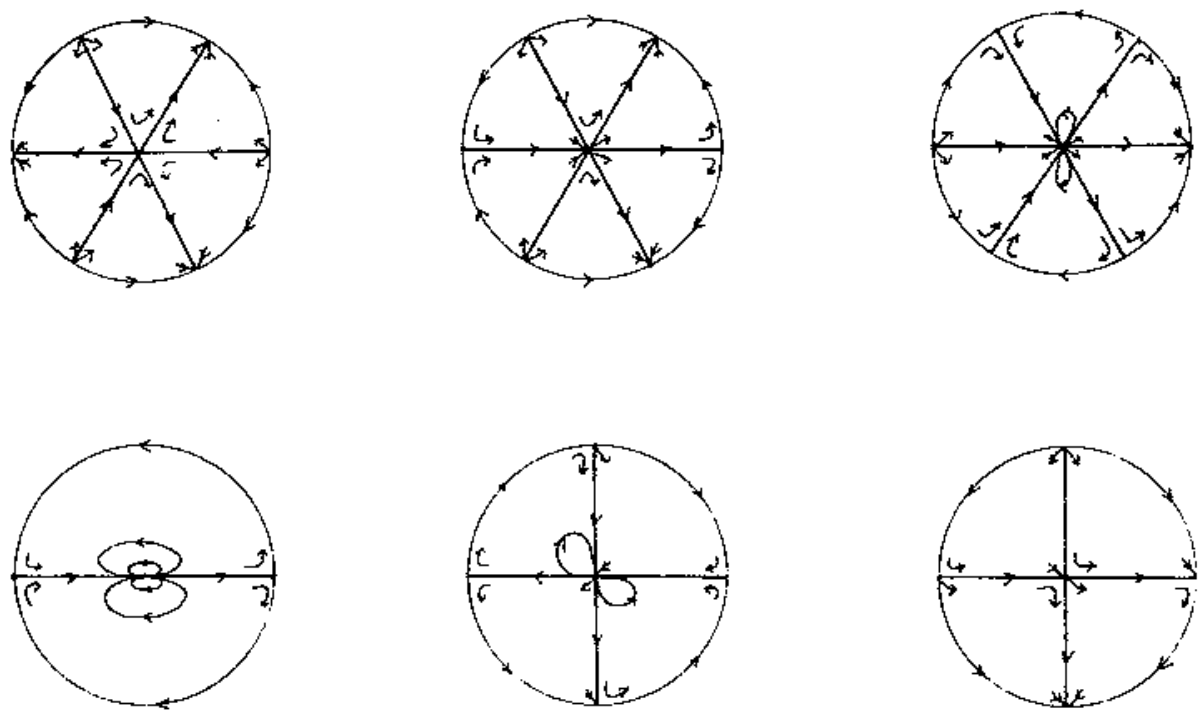

Figure 1.1. Phase portraits for homogeneous quadratic systems, topologically different from (b) of Figure 3.1.

From the following result we know that the only cases of the ones of Table 1.17 for which we can have limit cycles are (1) and (2).a.

Proposition 1.9. (See [C1], $[\mathrm{CJ}]$ ). Let $\gamma$ be a periodic orbit of a $Q S$ and $D$ the region bounded by $\gamma$. Then there exists an open set $U$ which contains $D$ such that $D$ contains exactly one singularity of the QS which is a weak focus, a focus or a center.

\section{Phase portraits for degenerate and linear QS1}

From Remark 6 of [CGL] a $Q S$ such that $g(x, y)=x Q_{2}(x, y)-y P_{2}(x, y) \equiv 0$ has no limit cycles. Degenerate and linear $Q S 1$ are such that $g(x, y) \equiv 0$ and hence they will not have limit cycles.

From Table 1.17, we know that the degenerate and linear $Q S 1$ are affine equivalents, scaling the variable $t$ if necessary, of one of the following types:

Degenerate: (III.e.3), (III.h.5), (V.e.3), (VIII.s.3);

Linear: (IV.e.4), (VIII. $e_{1} .3$ ), (VIII. $e_{2} .3$ ),

We can write them in the following form. 
Lemma 2.1. For a degenerate or linear QS1 there exists an affine transformation and a scaling of the variable $t$ which reduces it to one of the following systems:

$\begin{array}{lll}(D .1) & \dot{x}=y+x^{2}, & \dot{y}=-x+b y+x y \text { with }|b|<2 . \\ (D .2) \quad \dot{x}=y+x^{2}, & \dot{y}=x y . \\ (D .3) \quad \dot{x}=x^{2}-1, & \dot{y}=x+y+x y . \\ (D .4) \quad \dot{x}=x^{2}, & \dot{y}=y+x y . \\ (L .1) \quad \dot{x}=y, & \dot{y}=-x+b y \text { with } 0 \leq b<2 . \\ (L .2) \quad \dot{x}=x, & \dot{y}=b y \text { with } b \neq 0 \text { and }|b| \leq 1 . \\ (L .3) \quad \dot{x}=x, & \dot{y}=x+y .\end{array}$

Proof: Cases (D.1), (D.2), (D.4) and (L.3) follow from (III.e.3), (III.h.5), (VIII.s.3) and (VIII.e .3 ) respectively. The case (D.3) follows from (V.e.3) using the coordinates $x_{1}=x, y_{1}=-y / d-1$. If $|b| \leq 1$ the system (VIII.e $e_{\mathrm{I}} .3$ ) is (L.2). If $|b|>1$ then system (VIII. $e_{1} .3$ ) becomes into (L.2) by using the transformation $x_{1}=y, y_{1}=x, t_{1}=b t$. Sytem (IV.e.4) with plus sing, or with minus sign and $b \geq 2$ becomes into (L.2) or (L.3) writting the linear part of the system in the canonical Jordan and scaling the variables. System (IV.e.4) with minus sign and $0 \leq b<2$ is (L.1).

Theorem 2.2. The phase portrait of a degenerate or linear $Q S 1$ is homeomorphic (except, perhaps the orientation), to one of the separatrix configurations shown in Figure 2.1. Furthermore, sytems (D.1)-(D.4), (L.1)-(L.3) realize these configurations.

Proof: By Lemma 2.1 to show the theorem it is sufficient to draw the phase portraits of systems (D.1)-(D.4), (L.1)-(L.3). The expression of (D.1) in coordinates $x_{1}=-1+y+b x, y_{1}=x$ is $\dot{x}=x y, \dot{y}=1+x+b y+y^{2}$ with $-2<b \leq 0$ (taking $x_{1}=-x, t_{1}=-t$ if necessary). In the local chart $U_{1}$ (see [G], [S]) this system becomes $y^{\prime}=z(1+b y+z), z^{\prime}=z(-y)$. Hence phase portraits $D_{1}$ or $D_{2}$ of Figure 2.1 follow according to $b \neq 0, b=0$. System (D.2) writes as $x^{\prime}=z, z^{\prime}=$ $-2 z$, in the local chart $U_{2}$. The orbits of this system (after omitting the common factor $z$ ) are $z=-y^{2} / 2+k$. Therefore its phase portrait is like $D_{3}$ of Figure 2.1. The trajectories of system (D.3) are $y(x)=[(x-1) \log (|x-1| /|x+1|)-$ $-2 k(x-1)] / 4$, and $x= \pm 1$. Hence its phase portrait is given by $D_{4}$ of Figure 2.1. The phase portrait of system (D.4) is given by $D_{5}$ of Figure 2.1 because its trajectories are $y(x)=k x \exp (-1 / x)$ and $x=0$. The linear system (L.1) has a centre or a focus at the origin according to $b=0$ or $0<b<2$. Therefore, its phase portrait is like $L_{1}$ or $L_{2}$ of Figure 2.1 , respectively. The phase portrait of the linear system (L.2) is given by $L_{3}, L_{4}$ or $L_{5}$ of Figure 2.1 according to $0<b<1, b=1$ or $-1 \leq b<0$. Lastly, $L_{6}$ of Figure 2.1 gives the phase portrait of (L.3). 

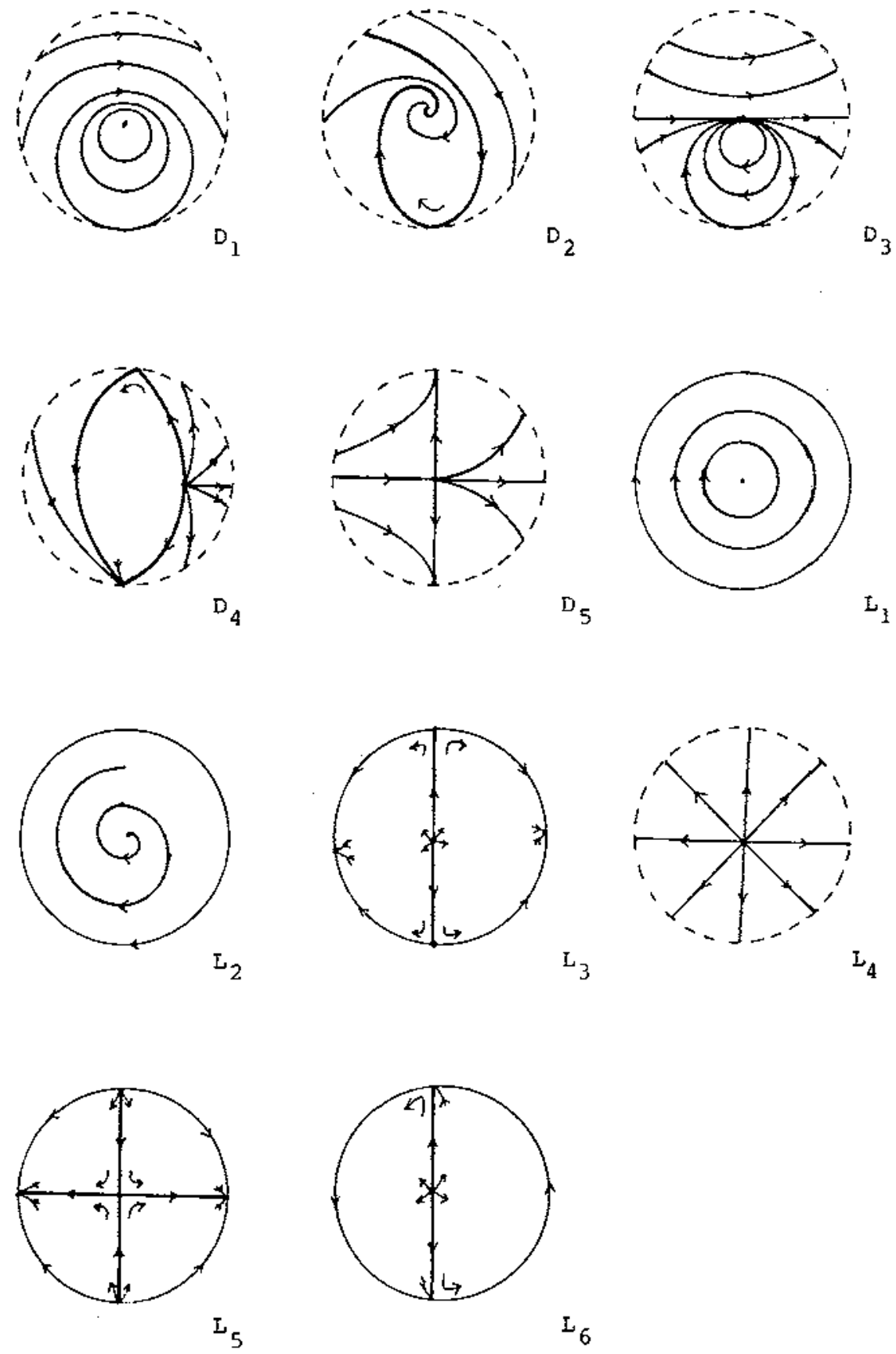

Figure 2.1. Phase portraits of QS1 either degenerate or linear. 


\section{Phase portraits for QS1 neither degenerate nor linear with the finite rest point of type $h$}

By Table 1.17 we must study the following cases:

(I.h.1), (I.h.2), (I.h.3), (I.h.6), (1.h.7), (I.h.8), (I.h.11), (I.h.12); (II.h.1), (II.h.2), (II.h.3), (II.h.4), (II.h.6), (II.h.7), (II.h.8), (II.h.9), (II.h.10); (III.h.1), (III.h.2), (III.h.3), (III.h.4); (IV.h.1), (IV.h.2), (IV.h.4), (IV.h.5); (VII.h.1), (VII.h.2), (VII.h.3).

Before begining the study case by case we shall give some general results that will be useful in order to short the proofs.

Proposition 3.1. The topological possibilities for a phase portrait of a $Q S 1$ of type $(A, a)$ where $A \in\{E, S\}$ and $a \in\{e, s, h\}$ are given in Figure 3.1.

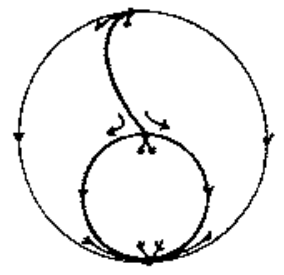

(a)

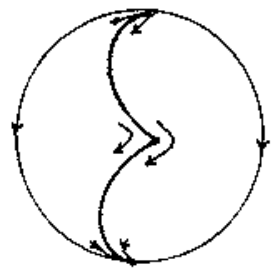

(b)

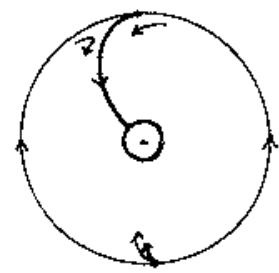

(c)

Figure 3.1. Topological possibilities for phase portraits of type $(A . a)$ where $A \in\{E, S\}$ and $a \in\{e, s, h\}$. The symbol $\rightarrow \odot$ denotes either a stable node or focus or stable or unstable focus on the interior of one or more limit cycles, the outhermost of which is externally stable.

Proof: It follows easily from Thcorems $E, S, H$ and the Poincaré-Hopf Theorem.

In Section 5 we shall see that the phase portrait given by (c) of Figure 3.1 will be either without limit cycles or with a unique stable limit cycle.

Proposition 3.2. (see [Y2]). Given a $Q S$ and a straight line $L$ connecting a finite critical point $M$ with an infinite critical point $N, L$ is either a trajectory or a line without contact (except $M$ ). In this later case, trajectories cut $\overline{N M}$ and $\overline{M N^{\prime}}$ (where $N^{\prime}$ is the diametrical opposite infinite critical point of $N$ ) in opposite direction.

Lemma 3.3. The local phase portraits of the singularity of type $H$ for a QS1 of type (II) with $n=0$ are those shown in Table 3.1. 
$m<0$.
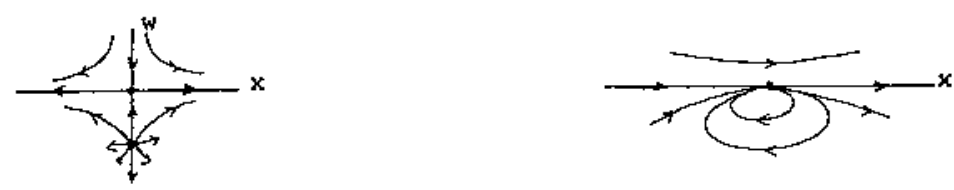

m=0, b- $\ell<0$.

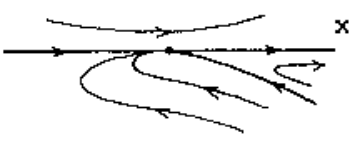

$m=b-2=0$,

$a<0$.
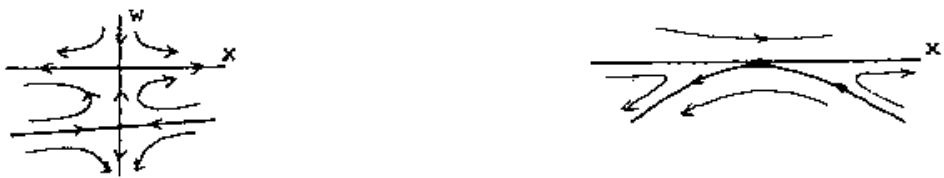

$m=b-\ell=0, a>0 \quad$ similar to case $m<0$.

m=0,b-2>0.

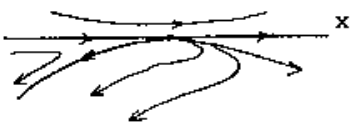

$0<\pi<1$

similar to case $m=b-\ell=0, a<0$.

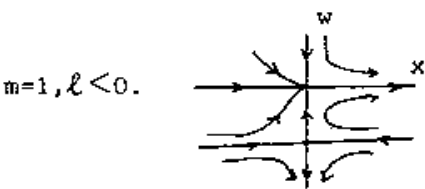

(*)

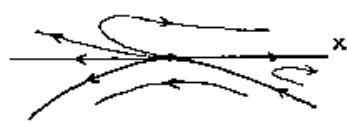

$m=1, \ell>0$.

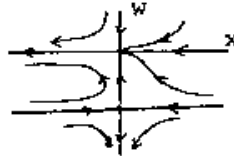

(*)

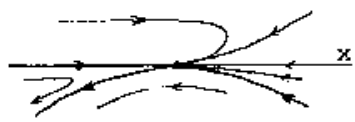

$1<\mathrm{m}<2$.

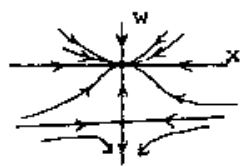

(*)

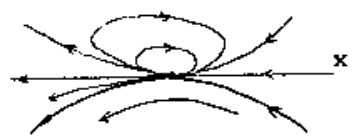

$\pi=2$.
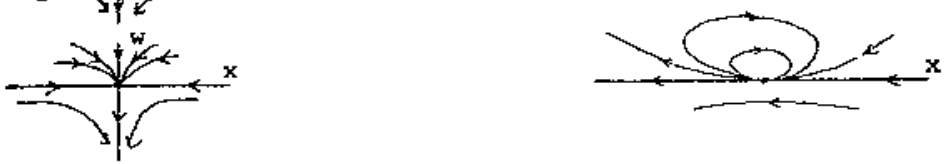

m $>2$.
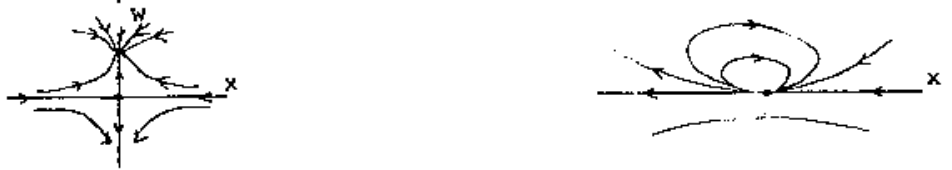

Table 3.1. Local phase portrait of the singularity of type $H$ for a $Q S 1$ of type (III). Note that in the cases (*) the infinity $z=0$ is not formed by separatrices. 
Proof: A QS1 of type (III) in the local chart $U_{2}$ writes in the form

$$
\begin{aligned}
& \dot{x}=z+(1-m) x^{2}-b x z-\ell x^{3}-a x^{2} z-d x z^{2}, \\
& \dot{z}=-m x z-b z^{2}-\ell x^{2} z-a x z^{2}-d z^{3} .
\end{aligned}
$$

After appling to this system two succesive changes of variables $x=x, z=$ $w_{1} x$ and $x=x, w_{1}=w x$ and omitting a common factor $x$, the system goes over to

$$
\begin{aligned}
& \dot{x}=(1-m) x-\ell x^{2}+x w-b x^{2} w-a x^{3} w-d x^{4} w^{2}, \\
& \dot{w}=(m-2) w+\ell x w-2 w^{2}+b x w^{2}+a x^{2} w^{2}+d x^{3} w^{3} .
\end{aligned}
$$

If $m \neq 2$ then this system has exactly two singularities on the $w$-axis, the points $(0,0)$ and $(0,(m-2) / 2)$. From. Theorems $E$ and $S$ we can study its nature. When $m=2$ the unique singularity is the origin. Now, by using Theorem $H$ the lemma follows.

In the hypotheses of Lemma 3.3, by studying the nature of the singularity of type $E$ (if it exists), we have the behaviour of the flow near infinity. It is showed in Figure 3.2 .
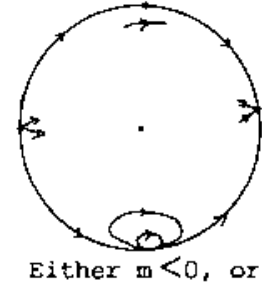

$\mathrm{m}=\mathrm{b}-\ell=0, \quad \mathrm{a}>0$.

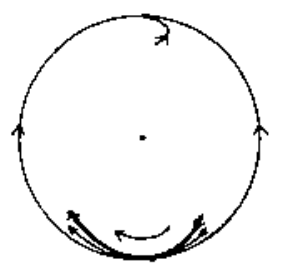

$\mathrm{m}=1, \ell>0$.

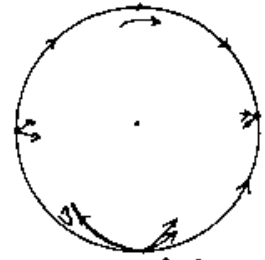

$\mathrm{m}=0, \mathrm{~b}-\ell<0$.

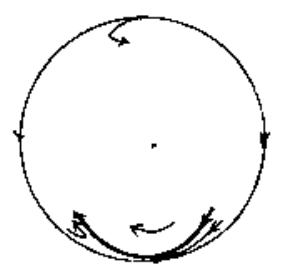

$m=1, \ell<0$.

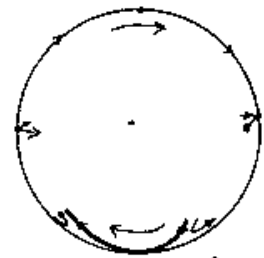

Either $\mathrm{m}=\mathrm{b}-\ell=0$, $a<0$, or $0<\mathrm{m}<1$.

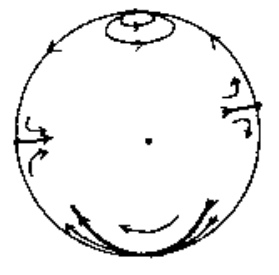

$1<\mathrm{m}<2$.

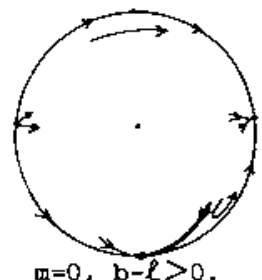

$m=0, b-\ell>0$.

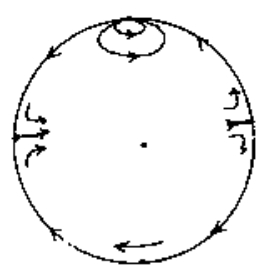

$\boldsymbol{x} \geq 2$.

Figure 3.2. Phase portraits of systems studied in Lemma 3.3 in a neighbourhood of infinity. 
Lemma 3.4. The local phase portrait of the singularity of type $H$ for the system $\dot{x}=y, \dot{y}=a x+b y+\ell x^{2}+m a y$ with $m \neq 0$ is shown in Figure 3.3 .

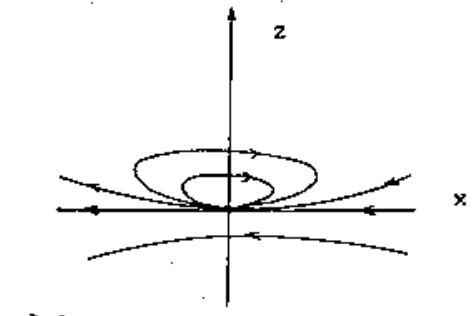

$\mathrm{m}>0$

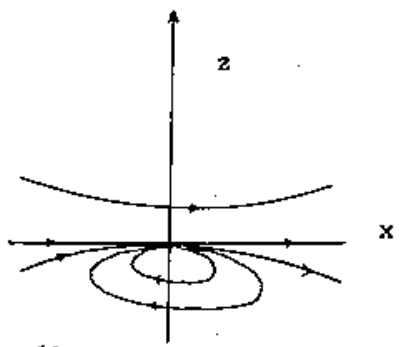

m $<0$.

Figure 3.3. Local phase portrait of the singularity of type $H$ for the system considered in Lemma 3.4 .

Proof: The system in the local chart $U_{2}$ gocs over to $\dot{x}=z-m x^{2}-b x z-$ $\ell x^{3}-a x^{2} z, \dot{z}=-m x z-b z^{2}-\ell x^{2} z-a x z^{2}$. We apply to this system the two succesive changes of variables $x=x, z=w_{1} x$ and $x=x, w_{1}=v x$. Therefore it is equivalent (after omitting a common factor $x$ ) to $\dot{x}=-m x-\ell x^{2}+x w-$ $b x^{2} w-a x^{3} w, \dot{w}=m w+\ell x w-2 w^{2}+b x w^{2}+a x^{2} w^{2}$. This system has two singularities on the $w$-axis, a saddle at $(0,0)$ and a node at $(0, m / 2)$. So the lemma follows.

We shall begin our study for system (VII) and will follow in the decreasing ordering.

Systems (VII.h.1), (VII.h.2) and (VII.h.3). These systems have the expression $\dot{x}=x^{2}, \dot{y}=x+\ell x^{2}+m x y+y^{2}$. In the coordinates $x_{1}=x$, $y_{1}=y+(m-1) x / 2$ these systems write as

$$
\dot{x}=x^{2}, \dot{y}=x+\ell^{\prime} x^{2}+x y+y^{2},
$$

where $\ell^{\prime}=\left(4 \ell-(m-1)^{2}\right) / 4$ and $\ell^{\prime}<0, \ell^{\prime}>0, \ell^{\prime}=0$ correspond with the systems (VII.h.1), (VII.h.2), (VII.h.3) respectively. By Theorem $H$ we have that the $(0,0)$ of $(3.1)$ is a saddle-note. In order to know if $x=0$ is a separatrix of this point we make two successive "blow ups": $x=w_{1} y, y=y ; w_{1} \doteq w y$, $y=y$ and system (3.1) transforms into (after omitting a common factor $y$ )

$$
\dot{y}=y+w y+w y^{2}+\ell w^{2} y^{3}, \quad \dot{w}=-2 w-2 w^{2}-2 \ell w^{3} y^{2}-w^{2} y
$$


that has two singularities on the $w$-axis. Since the $(0,0)$ is a saddle point we have that $x=0$ is a separatrix of $(0,0)$ of system $(3.1)$.

The point $(0,0)$ of the local chart $U_{2}$ is an attractor node. By Theorems $E$ and $S$ in the local chart $U_{1}$ there is a saddle and a node, no singularities, or one saddle-node if $\ell^{\prime}<0, \ell^{\prime}>0$ or $\ell^{\prime}=0$, respectively. From these facts and the study of the vector field given by $(3.1)$ on the axes we have pictures $h_{1}, h_{2}$ ((a) of Figure 3.1 ) and $h_{3}$ of Figure 3.4 according with $\ell^{\prime}<0, \ell^{\prime}>0$ or $\ell^{\prime}=0$, respectively.

System (IV.h.1). The equations of this system are $\dot{x}=y, \dot{y}=\ell x^{2}+m x y+$ $+n y^{2}$ with $n \ell \neq 0$ and $m^{2}-4 n \ell>0$. In coordinates $x_{1}=n x, y_{1}=n y$ it can be written as

$$
\dot{x}=y, \quad \dot{y}=\ell x^{2}+m x y+y^{2}
$$

where we can assume that $m \leq 0$ (with the change of variables $y_{1}=-y, x_{1}=x$, $t_{1}=-t$ if necessary). By Theorem $H$, the $(0,0)$ of $(3.2)$ is the reunion of two hyperbolic sectors. By Theorems $E$ and $S$, we obtain two different behaviours on the infinity according with $\ell>0$ or $\ell<0$. By studying vector field (3.2) on the axes and by Proposition 3.2 we obtain phase portraits $h_{4}$ and $h_{5}$ of Figure 3.4 , respectively.

System (IV.h.2). By Theorem $H$ and Proposition 3.1 the phase portrait of this system is (b) of Figure 3.1 or equivalently $h_{6}$ of Figure 3.4 .

System (IV.h.4). In the coordinates $x_{1}=m^{2} x / \ell, y_{1}=m^{3} y / \ell^{2}, t_{1}=\ell t / m$ this system can be written as

$$
\dot{x}=y, \quad \dot{y}=x^{2}+x y .
$$

By Theorem $H$, the finite singularity is the reunion of two hyperbolic sectors. By Lemma 3.4, the singularity $(0,0)$ of the local chart $U_{2}$ is the union of a hyperbolic sector with an elliptic one and the infinity, $z=0$, is formed by separatrices. From Theorem $S$ and the Poincaré-Hopf index Theorem we can prove that the singularity of type $S$ is a saddle-node. In short, taking into account the flow on the axes the phase portrait of (3.3) is like $h_{7}$ of Figure 3.4 .

System (IV.h.5). This system has the expression $\dot{x}=y, \dot{y}=x^{2}$ (after scaling the variables). Its integrals curves are $x^{3} / 3-y^{2} / 2=k$. So its phase portrait is homeomorphic to $h_{6}$ of Figure 3.4 .

Systems (III.h.I), (III.h.2) and (III.h.3). These systems can be written as $\dot{x}=y+x^{2}, \dot{y}=\ell x^{2}+m x y+n y^{2}$ with cither $n \neq 0, m^{2}-4 n \ell<0$ or $n \neq 0$, $m=\ell=0$. If $m=\ell=0$, using the changes $x_{1}=y, y_{1}=x$ and $x_{2}=n^{2} x_{1}$, $y_{2}=n y_{1}, t_{2}=t / n$ this system writes like a system of type (VII.h) that has 

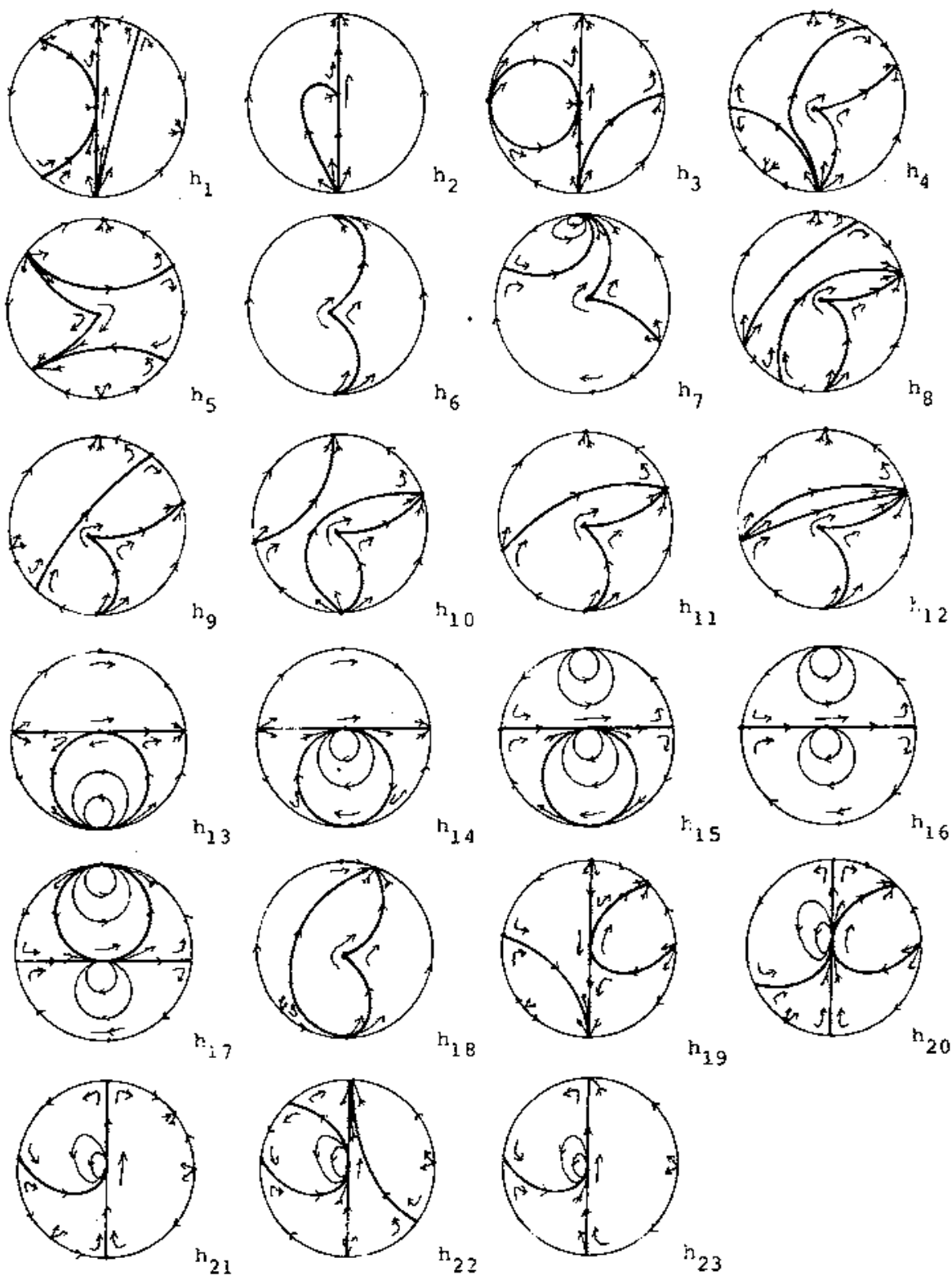

Figure 3.4. Phase portraits of QSI that have a finite critical point of type $h$ and whose infinity is neither degenerate nor linear. 
been already studied. In the other case the change of coordinates $x_{1}=x / n$, $y_{1}=y / n^{2}, t_{1}=n t$ writes our systems in the form

$$
\dot{x}=y+x^{2}, \quad \dot{y}=\ell x^{2}+m x y+y^{2} .
$$

By Theorem $H$, the finite singularity of this system is the union of two hyperbolic sectors. By Theorems $E$ and $S$, the behaviour of the vector field in a neighbourhood of infinity for systems (III.h.1), (III.h.2) and (III.h.3) is given by (a), (b) and (c) of Figure 3.5, respectively.
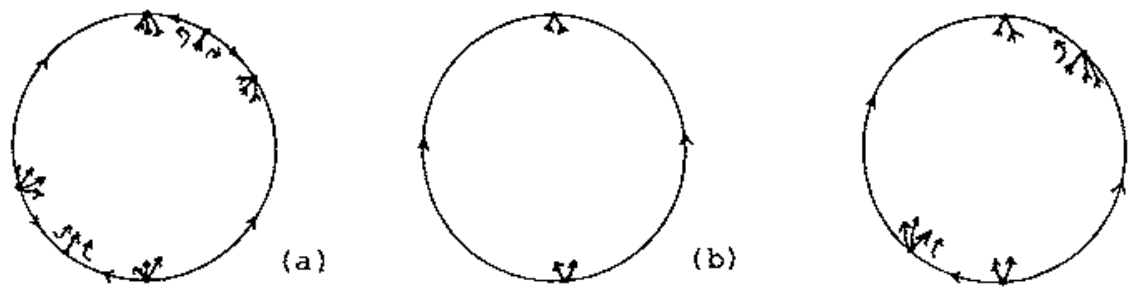

(c)

Figure 3.5. Behaviour in a neighbourhood of infinity of system (3.4).

The case (III.h.2) is already studied in Proposition 3.1. By Proposition 3.2 by taking the straight line connecting the $(0,0)$ with the saddle at infinity (resp. the saddle-node at infinity) in the case (III.h.1) (resp. (III.h.3)) and studying the vector field on the axes we can determine totally the behaviour of the separatrices of the origin. Their behaviour is showed in Figure 3.6.
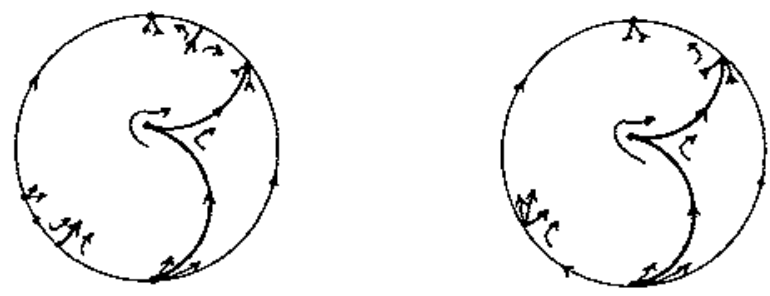

Figure 3.6. Separatrices of the origin of system (3.4). 
Hence the topological possibilities for the phase portrait of system (3.4) are $h_{8}, h_{9}, h_{10}, h_{11}$ and $h_{12}$ of Figure 3.4 . Note that $y=-m x-m$ is an invariant straight line by the fiow when $\ell=-m$ and it is a straight line without contact when $\ell \neq-m$. From this fact we have that the phase portraits of system (3.4) when $\ell=-m, m \in(-1,0) ; \ell=-m, m \in(-4,-1) ; m<-1 ; m=-1$ are $h_{8}$; $h_{9}, h_{10}$ and $h_{11}$ of Figure 3.4, respectively. New we must find a $Q S 1$ that has the phase portrait $h_{12}$ of Figure 3.4. We do not worried about it because this phase portrait will be the phase portrait of system (I.h.8).

System (III.h.4). This system has the expression

$$
\dot{x}=y+x^{2}, \quad \dot{y}=\ell x^{2}+m x y
$$

with $m \neq 1$ and either $m \neq 0$ and $\ell=0$ or $m=0$ and $\ell \neq 0$. Assume $m \neq 0$. Then by Theorem $H$ the singularity $(0,0)$ of $(3.5)$ is a saddle if $m<0$ and the union of a hyperbolic sector with an clliptic one if $m>0$. By using the same arguments that in the study of the finite singularity of systems (VII.h.I), (VII.h.2) and (VII.h.3), when $m>0$ we have that the invariant straight line $y=0$ is formed by separatrices only if $0<m \leq 2$. Therefore, by Lemma 3.3 it follows the phase portraits $h_{13}, h_{14}, h_{15}, h_{16}$ and $h_{17}$ of Figure 3.4 according to $m<0,0<m<1,1<m<2, m=2$ and $m>2$, respectively.

Now suppose $m=0$. Then system (3.5) can be written in the form

$$
\dot{x}=y+x^{2}, \quad \dot{y}=x^{2}
$$

For this system the origin is the union of two hyperbolic sectors (see Theorem $H$ ). So, by Lemma 3.3, and studying the flow on the $x$ and $y$ axes, we obtain that the phase portrait of (3.6) is like $h_{18}$ of Figure 3.4 .

System (II.h.1). This system can by written in the form $\dot{x}=x y, \dot{y}=$ $=d+a x+b y+\ell x^{2}+m x y+n y^{2}$, with $n \neq 0,1, \ell \neq 0, b^{2}-4 n d<0, a^{2}=4 \ell d$, $2 b \ell=m a$, and $m^{2}-4(n-1) \ell>0$. By using the change of coordinates $x_{1}=a|a|^{-1} x, y_{1}=m|m|^{-1}|a| a^{-1} y, t_{1}=m|m|^{-1}|a| a^{-1} t$, (where $m|m|^{-1}=1$ if $m=0$ ) we can assume that $a>0, m \geq 0$.

The finite singularity $(-a / 2 \ell, 0)$, by Theorem $H$, is tnion of two hyperbolic sectors. By Theorem $E$ and by studying the vector field on the straight lines $y=0, x=0$ (invariant), $x=-a / 2 \ell$, and the ones connecting the finite singularity and the saddle at infinity (if $n>1$ ) we obtain phase portraits homeomorphic to $h_{8}, h_{9}$, and $h_{5}$ of Figure 3.4 according with $n>1,0<n<1$ and $n<0$ respectively.

Systems (II.h.2), (II.h.7) and (II.h.9). These systems have been studied in Proposition 3.1. 
System (II.h.3). This system has the expression $\dot{x}=x y, \dot{y}=d+a x+b y+$ $+\ell x^{2}+m x y+n y^{2}$ with $n \neq 0,1, \ell \neq 0, m^{2}=4(n-1) \ell, b^{2}-4 n d<0, a^{2}=4 \ell d$, $2 b \ell=m a$. In the coordinates $x_{1}=a x / b^{2}, y_{1}=y / b, t_{1}=b t$ it can be written as $\dot{x}=x y, \dot{y}=1 /(4 n-4)+x+y+(n-1) x^{2}+(2 n-2) x y+n y^{2}$ with $n>1$. The finite singularity, by Theorem $H$, is union of two hyperbolic sectors. By Theorems $E$ and $S$ and by studying the vector field on the straight lines $x=0$ (invariant), $y=0, x=-1 /(2(n-1))$ and by Proposition 3.2 we obtain that the phase portrait of this system is homeomorphic to $h_{10}$ of Figure 3.4.

System (II.h.4). This system becomes

$$
\dot{x}=x y, \quad \dot{y}=x+x y+n y^{2},
$$

with $n \neq 0,1$ in the variables $x_{1}=m^{2} x / a, y_{1}=m y / a, t_{1}=a t / m$.

By Theorems $E$ and $S$ we can determine the behaviour of system (3.7) in a neighbourhood of infinity. By Theorem $H$, the origin is a saddle if $n<0$ and the union of a hyperbolic sector and an elliptic one if $n>0$. By using the same arguments that in the cases (VII.h.1), (VII.h.2) and (VII.h.3) we have that the invariant straght line $y=0$ is a separatrix if $n \geq 1$ and it is not a separatrix if $0<n<1 / 2$. Hence phase portraits of system (3.7) are $h_{19}, h_{20}, h_{21}$ and $h_{22}$ of Figure 3.4 according with $n<0,0<n<1 / 2,1 / 2 \leq n<1$ and $n>1$, respectively.

System (II.h.6). In coordinates $x_{1}=a|a|^{-1}(\ell / d)^{1 / 2} x, y_{1}=(1 /|d|)^{1 / 2} y$, $t_{1}=|d|^{1 / 2} t$ this system can be written like $\dot{x}=x y, \dot{y}=1+2 x+x^{2}$. This system has the invariant curves $x=0, y^{2}=x^{2}+4 x+2 \log |x|+k$. So its phase portrait is homeomorphic to $h_{9}$ of Figure 3.4.

System (II.h.8). In coordinates $x_{1}=a|a|^{-1} \ell^{1 / 2} d^{-1 / 2} x, y_{1}=d^{-1 / 2} y, t_{1}=$ $=d^{1 / 2} t$ it writes in the form $\dot{x}=x y, \dot{y}=1+2 x+m^{\prime} y+x^{2}+m^{\prime} x y+y^{2}$ with $m^{\prime} \neq 0$ and $\left|m^{\prime}\right|<2$. By using the new coordinates $x_{1}=x, y_{1}=-y, t_{1}=-t$ (if necessary) we can assume that $0<m^{\prime}<2$. By the same arguments that in the above cases we obtain that its phase portrait is homeomorphic to $h_{11}$ of Figure 3.4 .

System (II.h.10). This system can be studied in the same way that system (II.h.4) but with $n=1$. Its phase portrait is given by $k_{23}$ of Figure 3.4.

Before the study of system (I) case by case we shall give some general results about it.

Lemma 3.5. (i) System (I.h) with $\ell=0$ is affine equivalent to some system contained in types (II.h) or (VII.h).

(ii) System (I.h) with $\ell \neq 0$ has two hyperbolic sectors at the origin.

Proof: (i) follows by taking the changes of variables $x_{1}=y, y_{1}=m x+n y$ if $m \neq 0$, and $x_{1}=y, y_{1}=x, t_{1}=n t$ if $m=0$, respectively, and (ii) follows from Theorem $H$. 
Hence from now on we will assume that $\ell \neq 0$ for system (I.h).

Lemma 3.6. System (I.h.11) (with $\ell \neq 0$ ) can be transformed, by a change of variables plus a scaling of the time, into one of the following cases: (III.h), (II.h) or (I.h.S).

Proof: This system has the equations $\dot{x}=y-x^{2}+x y, \dot{y}=\ell x^{2}+m x y+y^{2}$ with $m \neq-1, \ell+m+1 \neq 0, m^{2}-4 \ell<0$. In the coordinates $x_{1}=x+(m+1) y / \ell_{1} y_{1}=$ $=y$ it writes as $\dot{x}=y+m x^{2}+(\ell-m(m+1)) x y / \ell, \dot{y}=\ell x^{2}+(-m-2) x y+(\ell+m+$ $+1) y^{2} / \ell$. If $\ell-m(m+1)=0$ in the new coordinates $x_{1}=m x, y_{1}=m y$ it writes like a system of type (III.h). If $\ell-m(m+1) \neq 0$ and $m=0$ this system writes like a system of type (II.h) in the coordinates $x_{1}=-x+1, y_{1}=y, t_{1}=-t$. Finally if $\ell-m(m+1) \neq 0$ and $m \neq 0$ in coordinates $x_{1}=(-m(m+1)+\ell) x / \ell$ $y_{1}=-((-m(m+1)+\ell) / \ell)^{2} y / m, t_{1}=-m \ell t /(-m(m+1)+\ell)$ we obtain $\dot{x}=y-x^{2}+x y, \dot{y}=(-m(m+1)+\ell) x^{2} / m^{2}+(m+2) x y / m+(m+1+\ell) y^{2} /(\ell-$ $-m(m+1))$ and since $(m+1+\ell) /(\ell-m(m+1)) \neq 1$ we have a system of type (I.h.3).

Systems (I.h.1) and (I.h.6) have at infinity singularities of types (E, E, E) and $(E, E, S)$ respectively. Hence from Lemma 3.5 (ii), Theorems $E, S$ and the Poincaré-Hopf index Theorem we have that in both cases the indices of the points at infinity are $(1,1-1)$ and, by consequence, these systems have always a saddle at infinity.

Lemma 3.7. None of the separatrices of the origin in systems (1.h.1) and (1.h.6) can coincide with the separatrices of the saddle points of these systems at infinity.

Proof: By theorem $H$ we know that the critical direction of the $(0,0)$ is $y=0$. Since $y=0$ is not an invariant straight line for these systems we know, by Proposition 3.2, that the straight line $L$ connecting the $(0,0)$ with the saddle point, at infinity is without contact for the flow of these systems. If we assume that a separatrix curve of the origin coincides with a separatrix at infinity of the saddle point then it should exist, at least, a contact point $p \in L$ and this fact is impossible (Figure 3.7 illustrates this claim).

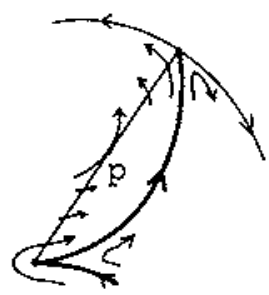

Figure 3.7. Local phase portrait that is not possible for $Q S$. 
Systems (I.h.1) and (I.h.6). From Lemma 4.7 the unique topological possibilities for the phase portraits of these systems are $h_{1}, h_{8}$ and $h_{9}$ of Figure 3.4. These phase portraits have been already realized.

Systems (I.h.2), (I.h.7) and (I.h.12). They follow from Proposition 3.1.

System (I.h.3). This system has the equations $\dot{x}=y-x^{2}+x y, \dot{y}=\ell x^{2}+$ $+m x y+n y^{2}$, with $\ell \neq 0, n \neq 0,1,2 m+4 \ell+1>0, m^{2}-4 n \ell+2 m+4 \ell+1=0$ and $2 n+m-1 \neq 0$. By using Lemma 3.5 (ii), Theorems $E$ and $S$ and Proposition 3.2 applied to the straight line connecting the $(0,0)$ with the saddle-node at infinity we obtain that its phase portrait is homeomorphic to $h_{10}, h_{11}$ or $h_{12}$ of Figure 3.4.

System (I.h.8). This system writes as $\dot{x}=y-x^{2}+x y, \dot{y}=-x^{2} / 4$. By Theorem $S$ and by studying the vector field on the straight lines $x=0, y=0$, $y=x / 2, y=x / 2-1 / 2$ we obtain that its phase portrait is homcomorphic to $h_{12}$ of Figure 3.4 , that is the one that we had not yet realized.

From Figure 3.4, it follows that in the study made in [JR] there are some omissions. For instance pictures $h_{15}, h_{17}$ and $h_{20}$ do not appear in their classification.

\section{Phase portraits for QSI neither degenerate nor linear with the finite rest point of type $s$ or e and without limit cycles}

In this section we shall study the phase portrait of $Q S 1$ neither degenerate nor linear with the finite rest point of type $s$ or $e$ and that can not have limit cycles. The systems with a singularity of type $e$ and that can have limit cycles will be studied in the following section.

We classify the systems studied in this section into the following cases:

(A) QS1 with some non-elementary singularity at infinity.

(B) $Q S 1$ with three elementary singularities at infinity.

(C) QSI with exactely two elementary singtalarities at infinity.

Note that we do not consider the systems with a unique elementary singularity at infinity because these systems have been already studied in Proposition 3.1 .

Case A. We subdivide these systems into two subcases: (A1). Systems of case $(A)$ and with the finite rest point of type $s$.

We must study systems (VII.s.1), (VII.s.2), (IV.s.5), (IV.s.6), (III.s.4) and (III.s.5).

System (VII.s.1). It has the equations $\dot{x}=x^{2}, \dot{y}=y+\ell x^{2}+m x y$, with $m \neq$ $\neq 1$. If $\ell \neq 0$ the we introduce the change of variables $x_{1}=x, y_{1}=x+(m-$ 1) $y / \ell$, and the system becomes into $\dot{x}=x^{2}, \dot{y}=x+y+m x y$ with $m \neq 1$. This 
system has the straight line $x=0$ invariant and the other solutions are given by

$$
y(x)=e^{-1 / x}|-x|^{m}\left(-\int_{t_{0}}^{-1 / x} t^{m-1} e^{-t} d t+k\right),
$$

where $t_{0}=0$ if $x<0$ and $t_{0}=-\infty$ if $x>0$. Drawing these solutions for $m<1$ or $m>1$ we obtain the phase portraits $E_{1}$ or $E_{2}$ of Figure 4.1, respectively.

When $\ell=0$ system (VII.s.1) has the straight lines $x=0$ and $y=0$ invariants, and the other solutions are $y(x)=k|x|^{m} e^{-1 / x}$. So, we have the phase portraits $E_{1}$ or $E_{3}$ of Figure 4.1 according to $m<1$ or $m>1$.

System (VII.s.2). In coordinates $x_{1}=x, y_{1}=y / \ell$ it writes $\dot{x}=x^{2}$, $\dot{y}=y+x^{2}+x y$. Its solutions are the straight line $x=0$ and

$$
y(x)=-x e^{-1 / x}\left(\int_{t_{0}}^{-1 / x} t^{-1} e^{-t} d t+k\right),
$$

where $t_{0}=0$ if $x<0$, or $t_{0}=-\infty$ if $x>0$. Therefore, its phase portrait looks like $E_{4}$ of Figure 4.1.

System (IV.s.5). In coordinates $x_{1}=\ell x, y_{1}=\ell y$ this system has the expression $\dot{x}=y, \dot{y}=y+x^{2}+m x y, m \neq 0$. By Lemma 3.4 the singularity of type $H$ is the union of an elliptic sector with a hyperbolic one, and the infinity in its neighbourhood is formed by two separatrices. By Theorem $S$ we obtain the local behaviours of the saddle-nodes at origin and at infinity. In short, by using the flow on the axes we obtain the phase portraits $E_{5}, E_{6}, E_{7}$ and $E_{8}$ of Figure 4.1 according to $m<-1, m=-1,-1<m<0$ and $m>0$, respectively.

System (IV.s.6). By using the same variables that in the above system we obtain that its expression is $\dot{x}=y, \dot{y}=y+x^{2}$. Since the infinite singularity is a node (by Theorem $H$ ) we obtain that its phase portrait is homeomorphic to the phase portrait (a) of Figure 3.1.

System (III.s.4). This system has the equations $\dot{x}=y+x^{2}, \dot{y}=y+\ell x^{2}+$ $+m x y$ where $m \neq 1$ and either $m \neq 0$ and $\ell=1$, or $m=0$ and $\ell \neq 1$.

Assume that $m \neq 0$. Then the finite singularity is a saddle if $m<0$ and an unstable node if $m>0$. Then, by Lemma 3.3, Figure 3.2 and by studying the flow on the axes we have the phase portraits $E_{9}, E_{10}, E_{11}$ and $E_{12}$ of Figure 4.1 according to $m<0,0<m<1,1<m<2$ and $m \geq 2$, respectively.

Now, suppose $m=0$. Then, the system becomes $\dot{x}=y+x^{2}, \dot{y}=y+\ell x^{2}$ where $\ell \neq 1$. By Lemma 3.3 , the singularity of type $H$ is a saddle-node an in its neighbourhood the infinity is formed by two separatrices. By Theorem $S$, the origin is a saddle-node and we know the local behaviour of its separatrices. In short, using the flow on the axes we obtain the phase portraits $E_{13}, E_{14}$, $E_{15}$ and $E_{16}$ of Figure 4.1 according to $\ell<-1, \ell=-1,-1<\ell<1$ and $\ell>1$, respectively. 

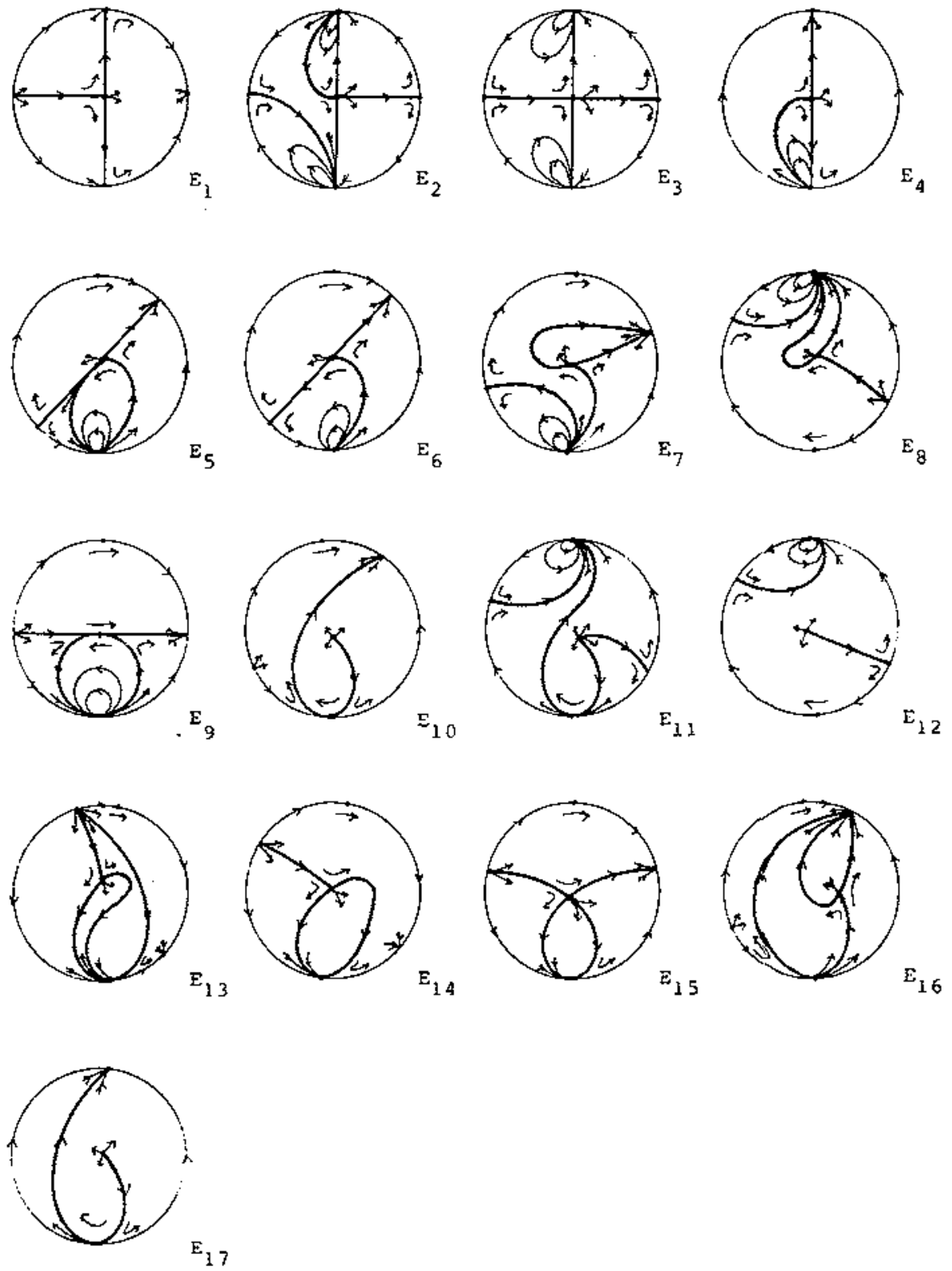

Figure 4.1. Phase portraits of case (Al). 
System (III.s.5). This system is given by the equations $\dot{x}=y+x^{2}, \dot{y}=$ $=y+x^{2}+x y$. The origin is an unstable node. Then, by Lemma 3.3 and by studying the flow on the axes we obtain that its phase portrait is like $E_{17}$ of Figure 4.1.

(A2). Systems of case (A) and with the finite rest point of type $e$.

We must study systems (VIII.e. 2$),\left(V I I I e_{2} .2\right),\left(V I I I e_{1}, 1\right),\left(V I I I e_{2} .1\right)$, (V.e.2), (V.e.1), (IV.e.3) and (III.e.1).

System (VIII.e 1.2 ). This system has the expression $\dot{x}=x, \dot{y}=b y+\ell x^{2}$ with $b \ell \neq 0$. We introduce the change $x_{1}=x, y_{1}=y / \ell$. Then, the system becomes $\dot{x}=x, \dot{y}=b y+x^{2}$ with $b \neq 0$. Its solutions are $y(x)=x^{2} /(2-b)+k|x|^{b}$ and $x=0$ if $b \neq 2$, or $y(x)=x^{2} \log |x|+k x^{2}$ and $x=0$ if $b=2$. So its phase portraits are like $E_{18},(c)$ of Figure 3.1 or $E_{19}$ of Figure 4.2 according to $b<0$, $0<b \leq 2$ or $2<b$. By reversing the orientation of all the trajectories in this last case we obtain the phase portrait of the bounded quadratic system omitted in [DP], (see also [CGL].)

System (VIIf.e 2.2 ). In the variables $x_{1}=\ell x, y_{1}=\ell y$ it converts into $\dot{x}=x, \dot{y}=x+y+x^{2}$. Since its solutions are $y(x)=x \log |x|+x^{2}+k x$ and $x=0$, its phase portrait is shown in (c) of Figure 3.1 .

System (VIII. $\mathrm{e}_{1} \cdot 1$ ). This system is given by $\dot{x}=x, \dot{y}=b y+\ell x^{2}+m x y$ with $b m \neq 0$. If $\ell \neq 0$ then the change of variables $x_{1}=m x, y_{1}=m^{2} y / \ell$ converts it to the form $\dot{x}=x, \dot{y}=b y+x^{2}+x y$ with $b \neq 0$. The solutions of this system are $x=0$ and

$$
y(x)=e^{b \log |x|} e^{x}\left(\int_{-\infty}^{\log x} e^{(2-b) t} e^{-e^{t}} d t+k\right) .
$$

So, its phase portrait is shown in $E_{20}$ or $E_{21}$ of Figure 4.2 according to $b<0$ or $b>0$.

When $\ell=0$ we introduce the coordinates $x_{1}=m x, y_{1}=x$ and the system has the equations $\dot{x}=x, \dot{y}=b y+x y$ with $b \neq 0$. Since its solutions are $x=0$ and $y(x)=k e^{x} e^{b \log |x|}$, we obtain the configurations $E_{22}$ or $E_{21}$ of Figure 4.2 according to $b<0$ or $b>0$.

System (VIIf.e 2.1 ). This system has the expression $\dot{x}=x, \dot{y}=x+y+$ $+\ell x^{2}+m x y$ with $m \neq 0$. We introduce the variables $x_{1}=m x, y_{1}=m(y+\ell x)$ and the system becomes $\dot{x}=x, \dot{y}=x+y+\ell(1-m) x^{2} / m+x y$. Its solutions are $x=0$ and

$$
y(x)=x e^{x}\left(\int_{-\infty}^{\log |x|} e^{-\mathbf{e}^{t}}\left(1+\ell(1-m) e^{t} / m\right) d t+k\right)
$$

So its phase portrait is like $E_{21}$ of Figure 4.2 . 

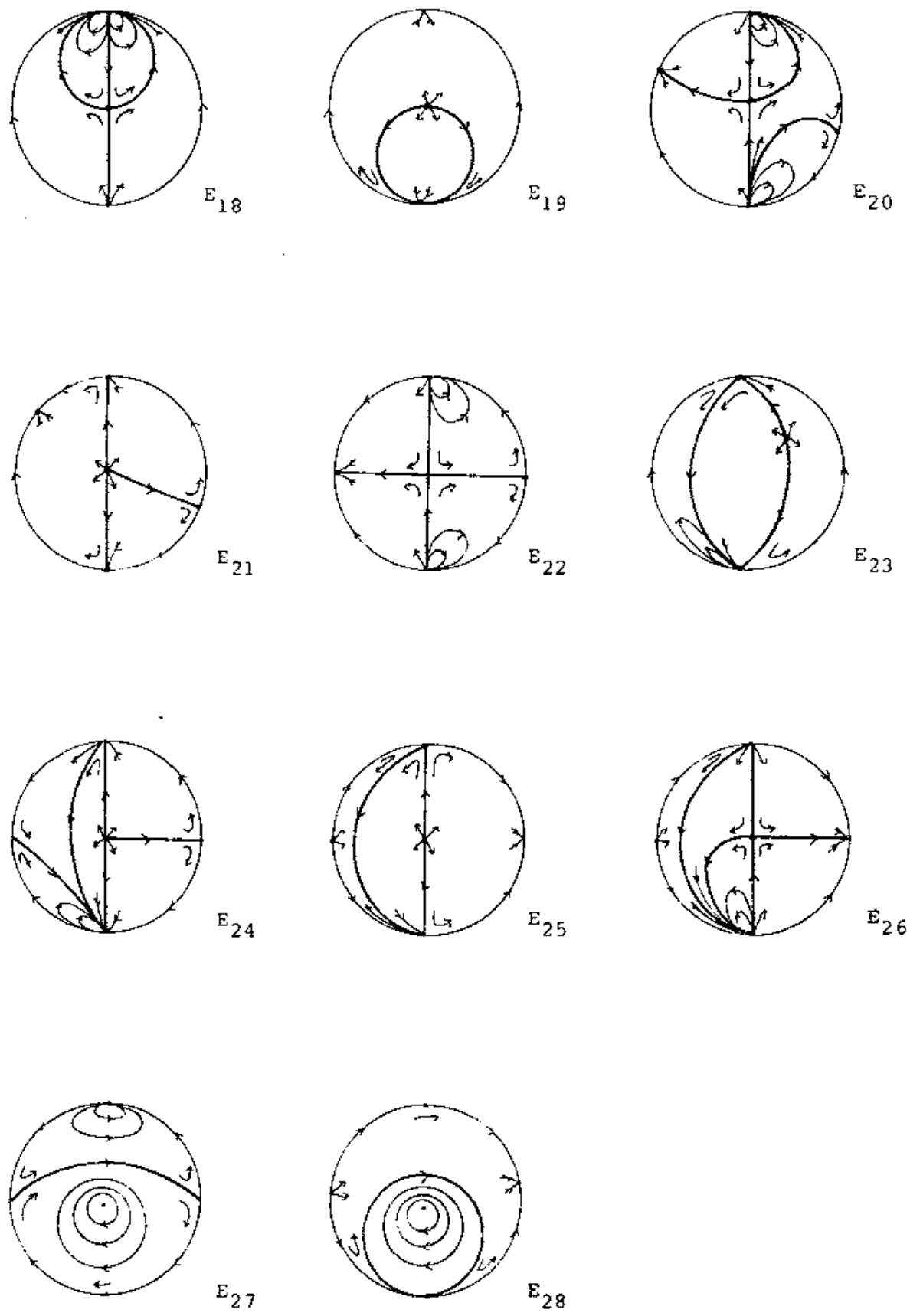

Figure 4.2. Phase portraits of systems (A2). 
System (V.e.2). This system is given by $\dot{x}=x^{2}-1, \dot{y}=d+y+\ell x^{2}+x y$ with $\ell \neq 0$ and $d+\ell \neq 0$. It goes over to $\dot{x}=x^{2}-1, \dot{y}=d^{\prime}+y+x^{2}+x y$ with $d^{\prime} \neq-1$, by using the transformation $x_{1}=x, y_{1}=y / \ell$. This system has the straight lines $x= \pm 1$ invariant and the other solutions are given by

$$
\begin{aligned}
y(x) & =2^{-1}(1-x)\left[2^{-1} d^{t} \log (|x-1| /|x+1|)+2^{-1} d^{\prime}(x+1)(x-1)-\right. \\
& \left.-\int_{t_{0}}^{1 /(2 \log (|x-1| /|x+1|))}\left(1+e^{2 t}\right)^{2} e^{-2 t}\left(1-e^{2 t}\right)^{-1} d t+k\right]
\end{aligned}
$$

where $t_{0}=0$ or $t_{0}=-\infty$ according to $x \in(-\infty,-1)$ or $x \in(-1,1) \cup(1, \infty)$. Drawing these solutions we obtain the phase portraits $E_{23}$ of Figure 4.2 and $E_{17}$ of Figure 4.1 according to $d^{\prime}+1<0$ or $d^{\prime}+1>0$.

System (V.e.1). The expression of this system is given by $\dot{x}=x^{2}-1$, $\dot{y}=d+m y+\ell x^{2}+m x y$ with $m \neq 0,1$ and $d+\ell \neq 0$. The change of variables $\dot{x}_{1}=x-1, y_{1}=y+\ell x /(m-1)+[d(m-1)-\ell(m-1)] /[(2 m)(m-1)]$ writes the system in the form $\dot{x}=2 x+x^{2}, \dot{y}=a^{\prime} x+2 m y+m x y$ with $a^{\prime}=-(d+\ell) / 2 \neq 0$. We can assume that $a^{\prime}>0$ using the change $x_{1}=x, y_{1}=-y$, if necessary. The solutions of this system are $x=0, x=-2$ and

$$
y(x)=|-x / 2|^{m}\left[-2 a^{\prime} \int_{t_{0}}^{\log (|x| /|x+2|) / 2}\left(1-e^{-2 t}\right)^{m-1} d t+k\right]
$$

where $t_{0}=0$ or $t_{0}=-\infty$ according to $x \in(-\infty,-2)$ or $x \in(-2,0) \cup(0, \infty)$. So, its phase portraits are like $E_{24}, E_{25}$ or $E_{26}$ of Figure 4.2 , according to $m>1,0<m<1$ or $m<0$.

System (IV.e.3). In coordinates $x_{1}=m x, y_{1}=m y$, it writes in the form $\dot{x}=y, \dot{y}= \pm x+b y+x y$, where $b \geq 0$. Suppose the plus sign in this system. Then the origin is a saddle. By Lemma 3.4 the singularity at infinity is the union at an elliptic sector with a hyperbolic one and the infinity is formed, in its neighbourhood, by two separatrices. By the Poincaré's index Theorem and Theorem $S$, the singularity of type $S$ is a node. So, the phase portrait of the system is given by $h_{13}$ of Figure 3.4 .

Assume now the minus sign in the system. If $b=0$ its solutions are $e^{y} e^{-x^{2} / 2}(y-1)=k$. So, its phase portrait is like $E_{27}$ of Figure 4.2 . When $b>0$ let $V(x, y)$ be the funtion $e^{y} e^{-x^{2} / 2}(y-1)$. Then $\dot{V}(x, y)=V_{x} \dot{x}+V_{y} \dot{y}=$ $=b y^{2} e^{y} e^{-x^{2} / 2} \geq 0$. Therefore, its phase portrait is given by $E_{12}$ of Figure 4.1 . In this picture, the origin is an unstable focus or node according to $0<b<2$ or $b \geq 2$, respectively.

System (III.e.1) with $m=0, \ell=b$. This system has the expression $\dot{x}=$ $=y+x^{2}, \dot{y}= \pm x+b y+b x^{2}$. In the coordinates $x_{1}=b x-y, y_{1}=x$ it writes like $\dot{x}=y, \dot{y}= \pm x+b y+n y^{2}$, with $n \neq 0$, and in the new variables $x_{1}=n x$, 
$y_{1}=n y$ it converts into $\dot{x}=y, \dot{y}= \pm x+b y+y^{2}$, where we can assume that $b \geq 0$. By Lemma 3.3, this last system with the plus sign has a phase portrait homeomorphic to $h_{13}$ of Figure 3.4.

If we consider this last system with the minus sign (the Liénard's system of degree two, see [LMP]) then, the function $V(x, y)=e^{-2 x}\left(x^{2}-y^{2}+1 / 2\right)$ is such that $\dot{V}(x, y)=V_{x} \dot{x}+V_{y} \dot{y}=-2 b y^{2} e^{-2 x} \leq 0$. So the phase portraits of this system with the minus sign are homeomorphic to $E_{28}$ or $E_{10}$ of Figures 4.2 or 4.1 respectively, according to $b=0$ or $b \neq 0$. Note that if $0<b<2$ the origin is a focus.

Case B. We subdivide these systems into the following subcases:

(B1) Systems $(B)$ with an elementary finite singularity of index 1 or -1 .

(B2) Systems $(B)$ with an elementary fnite singularity of index 0 . We separate them into:

(B2.a) Systems $(B 2)$ where indices of the infinite singularities are $(1,0,0)$.

(B2.b) Systems $(B 2)$ where indices of the infinite singularities are $(1,1,-1)$.

Now we begin the study of these subcases.

Case (BI). In this case, since it is very easy to do a study of all topological possibilities for the phase portraits of these systems, we summarize the results in the following two lemmas, which are given without proof. To prove them use the Poincaré-Hopf Theorem.

Lemma 4.1. The phase portrait of a QS1 with three elementary infinite critical points and the finite point of saddle type is homeomorphic to one of the phase portraits of Figure 4.3.
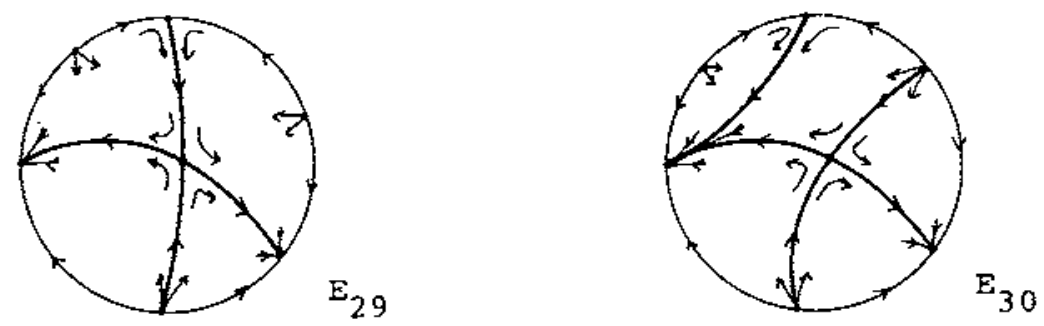

Figure 4.3. Phase portraits of systems $(B 1)$ with a saddle point as finite singularity.

Lemma 4.2. The phase portrait of a QSI with three elementary infinite critical points, the finite critical points of index +1 and without periodic orbits 
is homeomorphic to one of the phase portraits of Figure 4.4 .
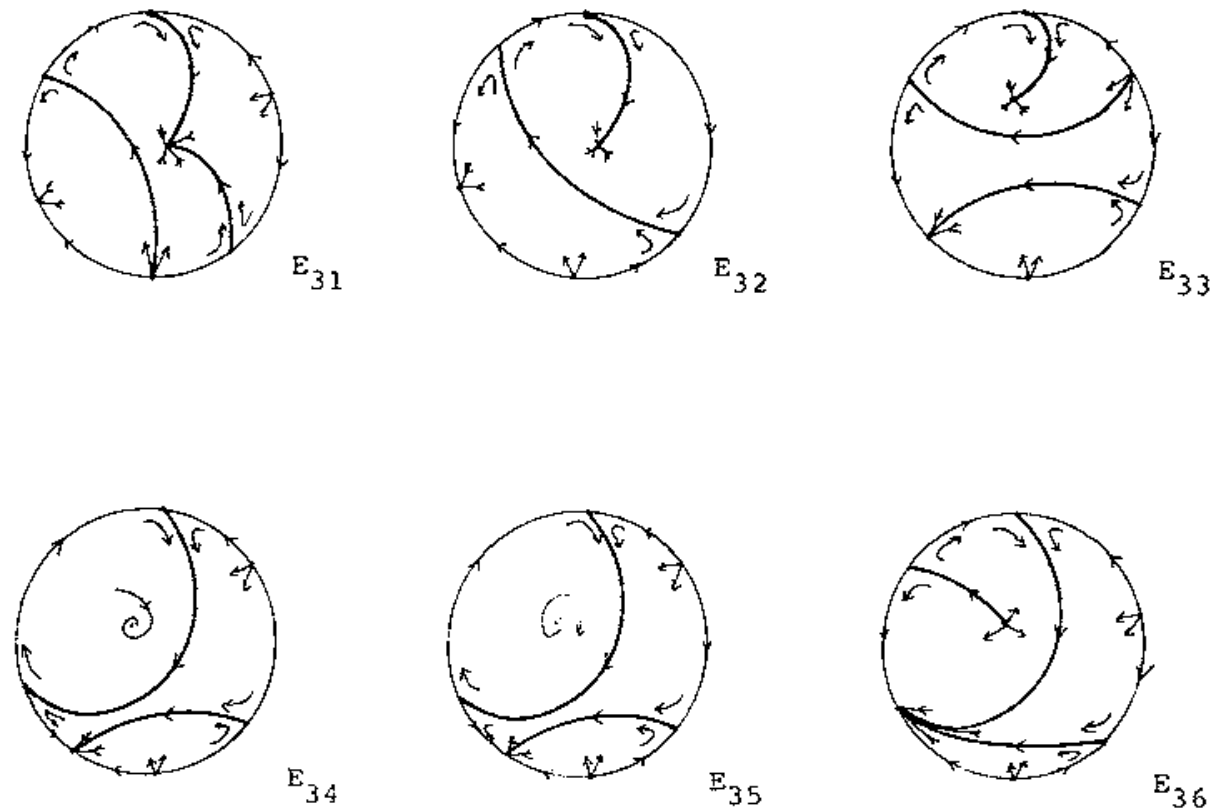

Figure 4.4. Phase portraits of systems (B1) with a point of index 1 as finite singularity.

Note that in this classification we do not consider the case in which the origin is a topological center because, by the results of Vulpe [V] we already know that there is only five different phase portraits of $Q S 1$ with a center. In fact two of these phase portraits have been already obtained in Section $2\left(D_{1}\right.$ and $L_{1}$ of Figure 2.1); and in Figure 4.2 we have two more phase portraits, $E_{27}$ and $E_{28}$. The remainder case will appear in Figure 5.7.

Now we must find $Q S 1$ that have the phase portraits of Figures 4.3 and 4.4 . Most of cases of Figure 4.4 will appear when we study the QS1 that can have limit cycles and so we will postpone this problem for the following section. The realization of cases of Figure 4.3 is given in Table 4.1 


\begin{tabular}{c|c|c}
\hline System & with & has the phase portfait \\
\hline (II.e.3) & $b=1, m \geq 0, d>0$ & $E_{29}$ \\
\hline (II.e.1) & $a=1, d<0, n<0$ & $E_{30}$ \\
\hline
\end{tabular}

Table 4.1. Realization of the phase portraits of Figure 4.3.

Case (B2.a). By Theorems $E$ and $S$, we know that we only can have indices $(1,0,0)$ at infinity when the singularities at infinity are of type $(E, S, S)$. So in this case we only must study the systems (VIII.s.1) and (IV.s.1).

System (VIII.s.1). In coordinates $x_{1}=x, y_{1}=n y$ its expression is given by $\dot{x}=x, \dot{y}=\ell x^{2}+m x y+y^{2}$ where $m^{2}-4 \ell>0$. If $\ell \neq 0$ then we can assume that $m \geq 0$ (by taking $x_{1}=-x$ if necessary). By Theorems $E$ and $S$, we obtain that the finite singularity is a saddle-nodc and the infinite singularities have indices $(1,0,0)$. Note that $x=0$ is an invariant straight line. If we consider the straight lines $y=m_{i} x$ where $m_{i}$ are the slopes of the infinite singularities, by Proposition 3.2 and Theorems $E$ and $S$, we obtain that its phase portraits are $E_{37}$ and $E_{38}$ of Figure 4.5 according with $\ell>0$ or $\ell<0$, respectively.
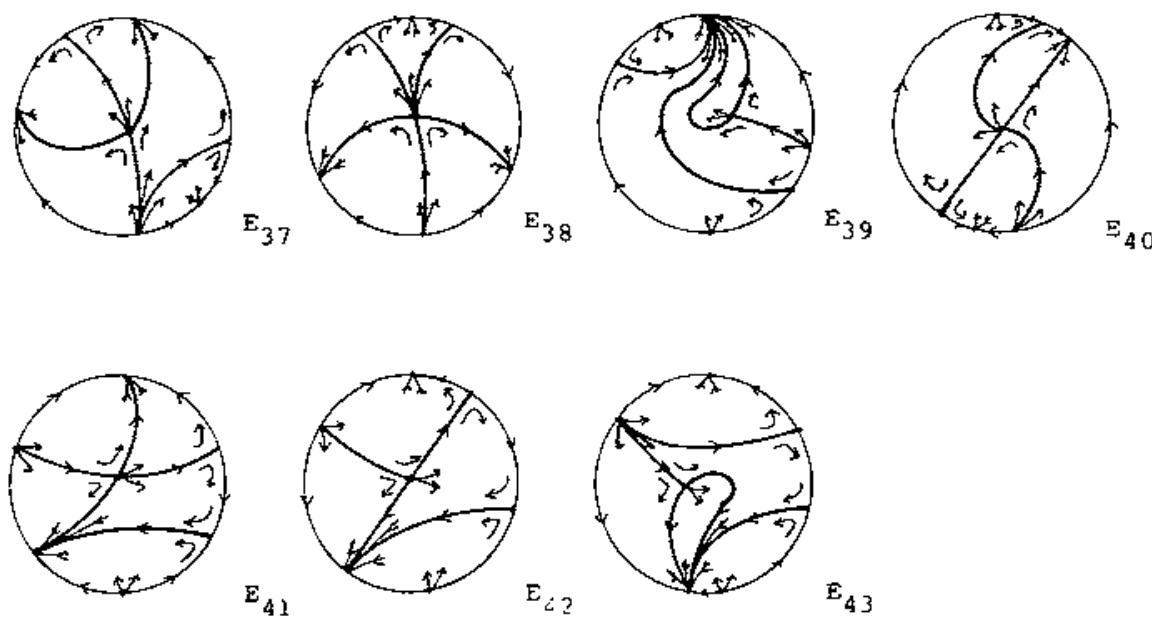

Figure 4.5. Phase portraits of systems (B2.a). 
If $\ell=0$, in coordinates $x_{2}=m x_{1}, y_{2}=y_{1}$ we obtain that its expression is $\dot{x}=x, \dot{y}=x y+y^{2}$. In this case $x=0$ and $y=0$ are invariant straight lines and it is easy to verify that its phase portrait is homeomorphic to $E_{38}$ of Figure 4.5 .

System (IV.s.1). In coordinates $x_{1}=x, y_{1}=n y, t_{1}=t$ the equations of this system are $\dot{x}=y, \dot{y}=y+\ell x^{2}+m x y+y^{2}$ with $m^{2}-4 \ell>0, \ell \neq 0$. The finite critical point is always a saddle-node whose separatrices approach to it in the directions of $y=0$ and $y=x$. The infinite critical points are always a node and two saddle-nodes (by Theorems $E$ and $S$ ). If we consider the vector field on the straight lines $x=0, y=0, y=m_{1} x, y=m_{2} x$ where $m_{1}$ and $m_{2}$ are the slopes of the infinite critical points, with $m_{1}>m_{2}$, then by Proposition 3.2 we can prove that the phase portraits of these systems are homeomorphic to $E_{39}$ if $\ell>0$ and $m>0$; to $E_{37}, E_{40}, E_{38}, E_{40}, E_{37}$ if $\ell>0, m<0$ and $m_{2}>1, m_{2}=1, m_{1}>1>m_{2}, m_{1}=1, m_{1}<1$, respectively; and to $E_{41}, E_{42}$ or $E_{43}$ of Figure 4.5 if $\ell<0$ and $m_{1}<1, m_{1}=1$ or $m_{1}>1$, respectively.

Case (B2.b). Instead of studying the systems cases by case we will do a topological classification of all the possibilities, and after we will find these phase portraits.

Lemma 4.3. The phase portrait of a QS1 with three elementary infinite critical points of indices $(1,1,-1)$ and a finite critical point of saddle-node type is homeomorphic to one of the phase portraits of Figure 4.6 .
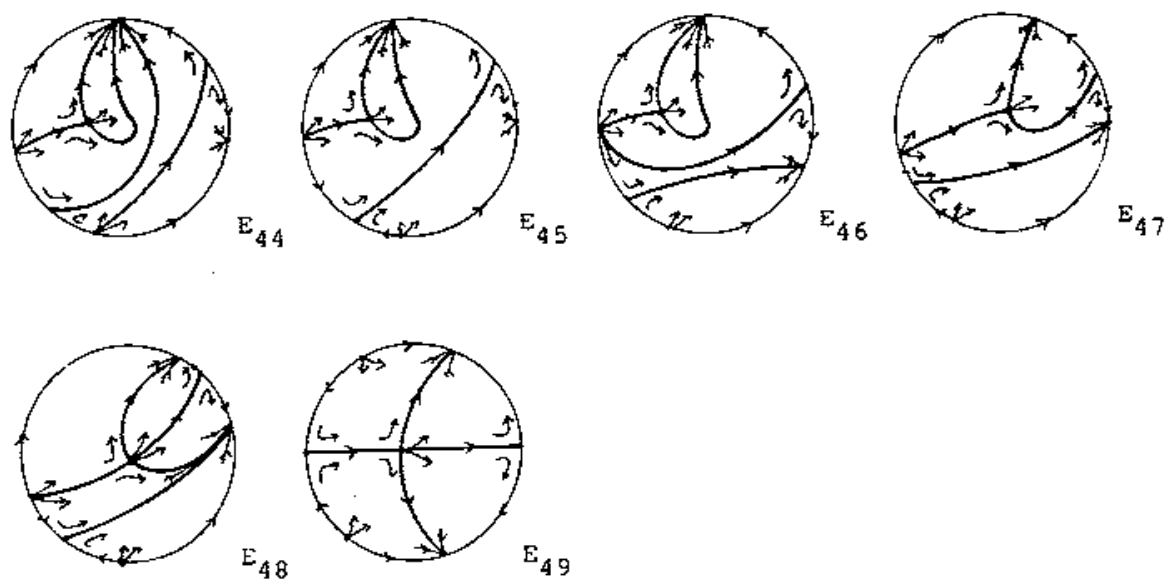

Figure 4.6. Phase portraits of systems $(B 2 . b)$. 
Proof: By making a linear change of coordinates plus a translation we can assume that the expression of the $Q S 1$ is given by $\dot{x}=L x^{2}+M x y+N y^{2}$, $\dot{y}=y+\ell x^{2}+m x y+n y^{2}$. If $L \neq 0$ we can assume that $L=1$ and $\ell$ is either 1 or 0 (in coordinates $x_{1}=L x$ and either $y_{1}=L^{2} y / \ell$ or $y_{1}=y$, respectively). If $L=0$, since the origin must be a saddle-node, $M$ must be zero and we have $\dot{x}=N y^{2}, \dot{y}=y+\ell x^{2}+m x y+n y^{2}$; again we can assume that $N=1$ and either $\ell=1$ or $\ell=0$. Hence, since $L=1$ or, $L=0$ and $N=1$, we have that the phase portrait in a neighbourhood of $(0,0)$ of our QS1 is one of the phase portraits given in Figure 4.7 where we have represented also the vector field on the axes.

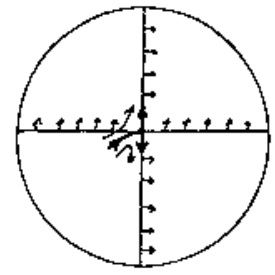

(a) $\ell=1, N>0$

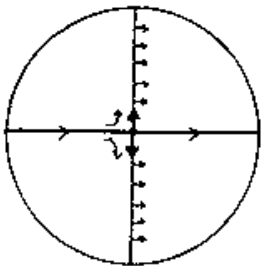

(d) $l=0, N>0$

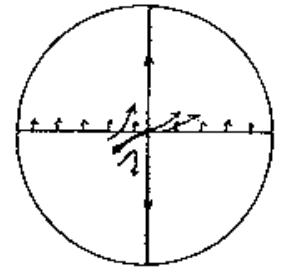

(b) $\ell=1, \quad N=0$

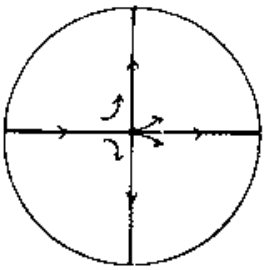

(e) $\ell=0, N=0$

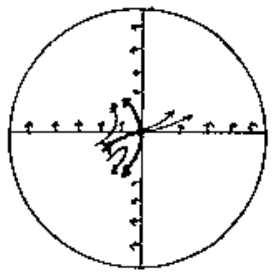

(c) $\quad l=1, N<0$

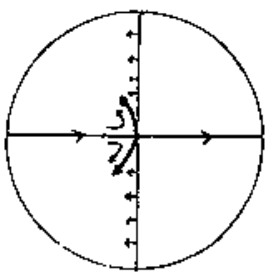

(f) $\ell=0, N<0$

Figure 4.7. Possible local behaviours of a saddle-node.

Now, we shall study every one of these cases, taking into account that the indices of the infinite singularities are $(1,1,-1)$ and by using Proposition 3.2. Consider for instance case $(a)$. By Proposition 3.2 there are no critical points at infinity for our system on the directions given by the straight lines $x=0$ and $y=0$. Since there are exactely three inifinite singularities and by taking the vector field on the axes we have that this case can be divided into the following two subcases:

(a1) The QS1 has an infinite singularity in the first quadrant and it has two infinite singularities in the second one.

(a2) The QS1 has three infinite singularities in the first quadrant. 
We study now the subcase (a1). The $\alpha$-limit of $C_{1}$ must be $p^{\prime}$ (see Figure 4.8). Hence the $\omega$-limit of $C_{3}$ is $r$. The $\omega$-limit of $C_{2}$ can not be $r^{\prime}$ because this point could not have hyperbolic sectors (look at the vector field on the straight line $r^{\prime}$ or ) and by consequence the $\omega$-limit of $C_{2}$ is $p$. The point $q$ must have a stable separatrix of two hyperbolic sectors (this is because the points with $\omega$-limit either $p$ or $r$ in the interior of the closed curve opqrp are open sets). Hence $q^{\prime}$ also must have an unstable separatrix, and the phase portrait is the one of Figure 4.8 (i.e. it is $E_{48}$ of Figure 4.6 ).

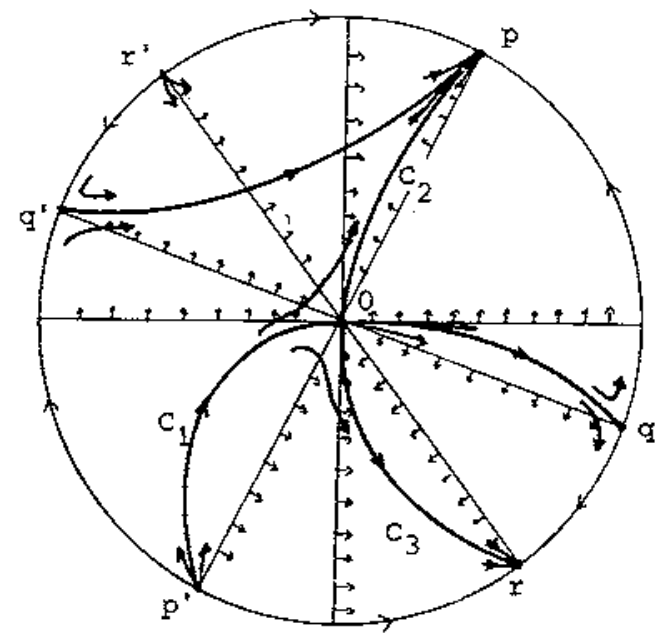

Figure 4.8. Phase portrait of a system $(B 2 . b)$ when it satisfies the hypotheses (a1).

If we use the same kind of arguments for the other cases and subcases we obtain $E_{44}, E_{45}, E_{46}, E_{47}$, and $E_{48}$ of Figure 4.6 for the subcase (a2) of Figure $4.7, E_{49}$ of Figure 4.6 in the case (e), and there are no possible phase portraits for the remainder local behaviours of Figure 4.7 .

In order to find $Q S 1$ that have the phase portraits of Figure 4.6 we consider system (III.s.1). It is easy to show that this system when $\ell=0, n<0$ and $m>1$ has phase portrait $E_{49}$ of Figure 4.6. In order to obtain all the other cases we shall study system (III.s.1) under the condition $\ell>1$. The phase portraits that we shall obtain are summarized in Table 4.2 . 


\begin{tabular}{c|c|c}
\hline System & with & has the phase portrait \\
\hline (III.s.1) & $n>0, y_{-}<-m / n<y_{+}$ & $E_{44}$ \\
\hline (III.s.1) & $n>0,-m / n=y_{+}$ & $E_{25}$ \\
\hline & $n>0$, and for some & $E_{46}$ \\
(III.s.1) & values of $\ell, m, n$ such & $E_{47}$ \\
& that $-m / n>y_{+}$ & $E_{48}$ \\
\hline (III.s.1) & $n<0, \ell=0, m>1$ & $E_{43}$ \\
\hline
\end{tabular}

Table 4.2. Realization of the phase portraits of Figure 4.6.

Consider system (III.s.1) with $\ell>1$, that is $\dot{x}=y+x^{2}, \dot{y}=y+\ell x^{2}+$ $+m x y+n y^{2}$, with $n \neq 0, m^{2}-4 n(\ell-1)<0,(m-1)^{2}-4 n \ell>0$, and $\ell>1$. The finite critical point is a saddle-node with critical directions $y=0$ and $y=x$. The critical points at infinity are a stable node in the $(0,0)$ of the local chart $U_{2}$, a saddle point on the point of $U_{1}$ corresponding to the direction of the straight line $y=y_{+} x$, and a stable node corresponding to the direction of the straight line $y=y_{-} x$ where $1<y_{-}<y_{+}$and $y_{ \pm}=\left(-(m-1) \pm\left((m-1)^{2}-4 n l\right)^{1 / 2} /(2 n)\right.$. The straight line $y=y_{+} x+\left(y_{+}^{2}-y_{+}\right) /\left(m+2 n y_{+}\right)$is an invariant straight line if $y_{+}=-m / n$ and a straight line without contact if $y_{+} \neq-m / n$. This fact allow us to determine that the phase portraits for our system when $y_{-}<-m / n<y_{+}$ (resp. $-m / n=y_{+}$) is homeomorphic to $E_{44}$ (resp. $E_{45}$ ) of Figure 4.6. When $y_{+}<-m / n$ we have that only the three phase portraits $E_{46}, E_{47}$ and $E_{48}$ are topologically possible. Note that in the space of parameters $\ell, m, n$, the subset $K=\left\{(\ell, m, n) \in \mathbf{R}^{3}: \ell>1, m^{2}-4 n(\ell-1)<0,(m-1)^{2}-4 n \ell>0\right\}$ is a connex set. So if we find two values of $(\ell, m, n) \in K^{r}$ such that the phase portraits of their associated systems are $E_{45}$ and $E_{48}$ we will have proved that the phase portraits $E_{46}$ and $E_{47}$ exist some values of $(\ell, m, n)$ belonging to $K$.

Two points of $K$ that have the phase portraits $E_{48}$ and $E_{45}$ are, for instance, $k=(2.256,-2.8,1.6)$ and $(4,-2,1 / 2)$ respectively. In order to prove that the phase portrait associated to the point $k \in K^{\prime}$ is $E_{48}$ we can consider the vector field on the straight line $y=x / 2$ (see Figure 4.9). 


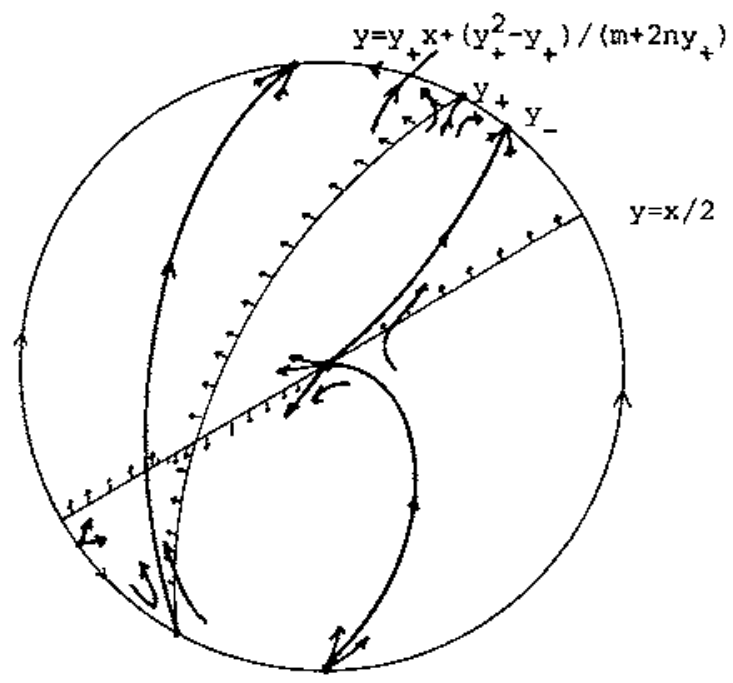

Figure 4.9. This $Q S 1$ has the phase portrait $E_{48}$ of Figure 4.6 .

Case (C). We subdivide these systems in the following subcases:

$(C 1)$ Systems $(C)$ with the elementary finite singularity of indices \pm 1 . $(\mathrm{C} 2)$ Systems $(C)$ with the elementary finite singularity of index 0 .

Case (C1). We do the same study that in the case $(B)$ and we obtain the following result.

Lemma 4.4. (i) The phase portrait of a QSI with elementary infinite critical points and the finite critical point of index +1 and without periodic orbits is homeomorphic to one of the phase portraits of Figure 4.10.

(ii) There are no QS 1 with two elementary infinite critical points and a finite critical point of index -1 .

Note that (ii) of the above lemma follows from the Poincaré-Hopf Theorem and Theorems $E$ and $S$.

The realization of some phase portrait of Figure 4.10 will be done in the following section. In fact not all the phase portraits of this Figure will exist for QS1. 

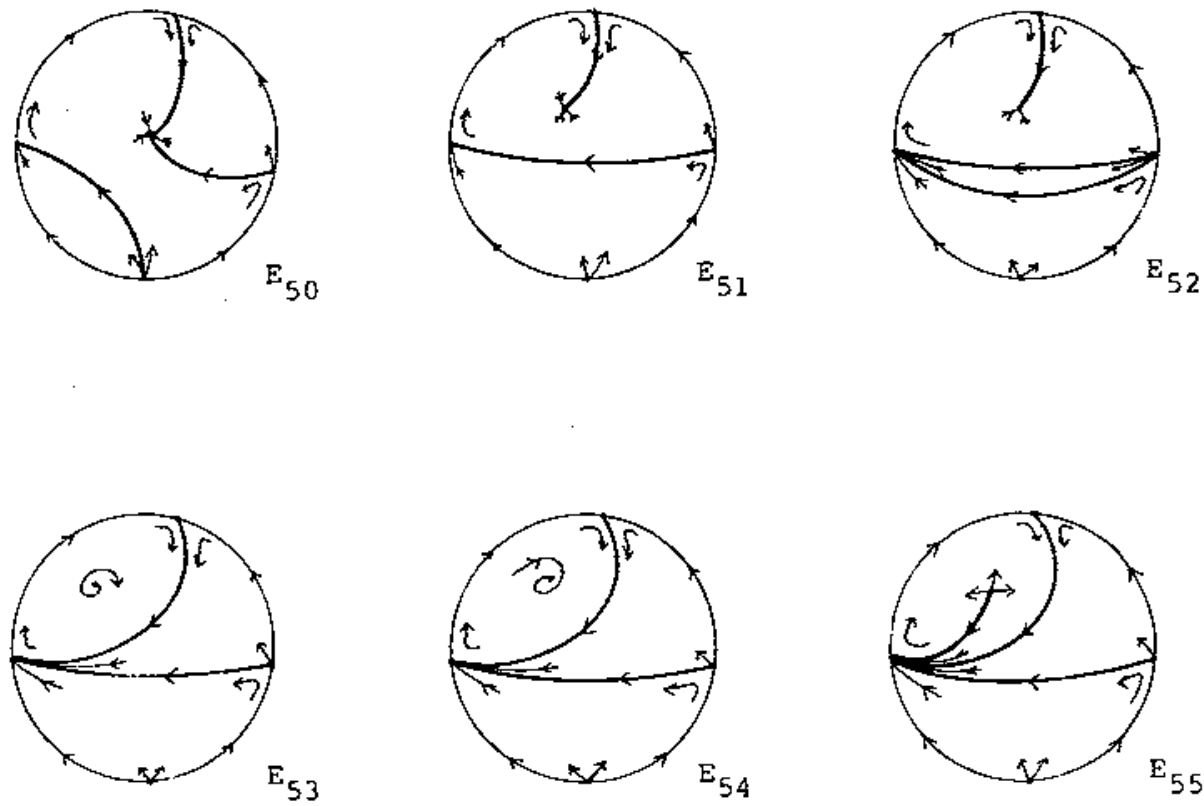

Figure 4.10. Possible phase portraits of systems (C1). We shall prove in the following section that not all these pictures are realizables for $Q S I$.

Case (C2). The following lemma and table are equivalent to Lemma 4.3 and Table 4.2 of Case $(B 2 . b)$.

Lemma 4.5. The phase portrait of a $Q S 1$ with two elementary infinite critical points and a finite critical point of saddle-node type is homeomorphic to one of the phase portraits of Figure 4.11 .

Proof: By using the same arguments that in the proof of Lemma 4.3 we obtain Figure 4.7. Now, we must consider all the possibilities taking into account Proposition 3.2. These possibilities are showed in Figure 4.11. Cases (b), $(c),(e)$ and $(f)$ of Figure 4.7 give no possible phase portraits. Case $(d)$ gives the phase portraits $E_{65}$ and $E_{66}$ of Figure 4.11 and the remainders follow from case $(a)$ of Figure 4.7 .

The phase portraits of Figure 4.11 can be realized by studying systems (II.s.13) and (III.s.3). The results obtained are summarized in Table 4.3 and the way to study system (III.s.3) when $n>0$ and $m<-1$ or when $n<0$ 
and $1>m>-1$ is equivalent to the way in which we have studied system (III.s.1), to realize case $(B 2 . b)$.

When $n<0$, if we consider the phase portraits of the QS1 associated to the points $\left.(\ell, m, n)=\left(-(m-1)^{2} / 1.2\right), m,-0.3\right)$ we have that they are homeomorphic to $E_{57}$ when $m=-1$ and to $E_{60}$ when $m=0.8$. The study when $n>0$ is similar. Furthermore we can consider it like the boundary of system $(M Y . s .1)$ studied in case $(B 2 . b)$ and for the values $n=1.6$ and $m=-2.8$ (that are the same values considered in the study on systems (III:s.1)) its phase portrait is homeomorphic to $E_{65}$ of Figure 4.11.

\begin{tabular}{c|c|c}
\hline System & with & has the phase portrait \\
\hline (III.s.3) & $n<0, m<-1$ & $E_{56}$ \\
\hline$(I I . s .3)$ & $n<0, m=-1$ & $E_{57}$ \\
\hline & $n<0 ;$ some values & $E_{58}$ \\
$(I I . s .3)$ & of $-1<m<1$ & $E_{59}$ \\
& & $E_{60}$ \\
\hline$(I I I . s .3)$ & $n>0,-1<m<1$ & $E_{61}$ \\
\hline$(I I I . s .3)$ & $n>0, m=-1$ & $E_{62}$ \\
\hline & $n>0 ;$ some values & $E_{63}$ \\
$(I I I . s .3)$ & of $m<-1$ & $E_{64}$ \\
\hline$(I I . s .13)$ & & $E_{65}$ \\
\hline
\end{tabular}

Table 4.3. Realization of the phase portraits of Figure 4.I1. 

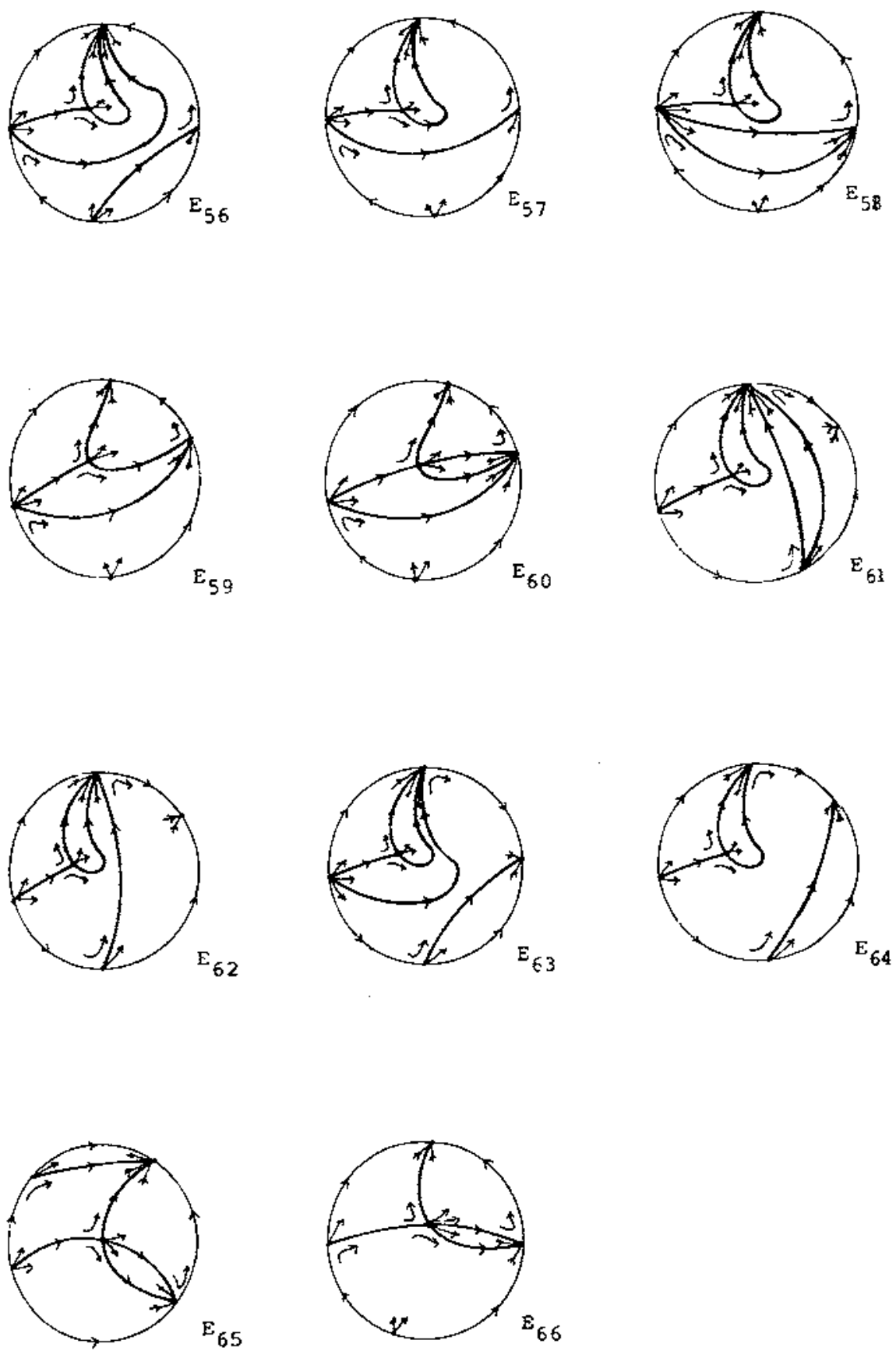

Figure 4.11. Phase portraits of systems (C2). 


\section{Phase portraits for QS1 that can have limit cycles}

Before a study case by case of the phase portraits of the QS1 that can have limit cycles we shall give their expressions in a more suitable way.

Theorem 5.1. A QS1 that can have limit cycles can be writen in one of the following forms:

(a) $\dot{x}=y, \dot{y}=-x+b y+x y+n y^{2}$ with $n<0$;

(b) $\dot{x}=y+x^{2}, \dot{y}=-x+b y+(\ell+b) x^{2}+m x y$ with $\ell^{2}-4 m<0$ and $\ell+b \leq 0$. Furthemore, if $m=1$ then $\ell+b \neq 0$;

(c) $\dot{x}=y+x y, \dot{y}=-x+b y+m x y+n y^{2}$ with $m<0,(b-m)^{2}-4 n<0$; $\ell=-1, m=0$

(d) $\dot{x}=y+p x^{2}+x y, \dot{y}=-x+b y+(\ell+b p) x^{2}+(m+b) x y$ with $p>0$,

(e) $\dot{x}=y+p x^{2}+x y, \dot{y}=-x+b y+(\ell+b p) x^{2}+(m+b) x y$ with $p>0$, $(1+\ell)^{2}-4 p m<0$.

Proof: By Section 4 and Table 1.17 we must consider only cases (I.e.4), (I.e.5), (I.e.6), (II.e.1), (II.e.9), (III.e.1) with $m \neq 0$, (III.e.2) and (IV.c.1), with a focus or a center at the origin, see Proposition 1.9. Note that the systems (II.e.3), (II.e.4) and (II.e.5) of Table 1.17, have the unique finite singularity on the invariant straight line $x=0$, so they can not have limit cycles.

System (IV.e.1) in coordinates $x_{1}=-m|n| \tau / n, y_{1}=m y, t_{1}=-|n| t / m n$ writes in the form (a).

System (III.e.1) with $m \neq 0$ and (III.e.2) write in the form (b), putting $\ell+b$ instead of $\ell$, and taking the variables $x_{1}=-x, y_{1}=y, t_{1}=-t$ if necessary.

We can assume that $a=m=1$ in systems (II.e.1) and (Y.e.9). In the new coordinates $x_{1}=-(x+d) / d, y_{1}=y / d^{1 / 2}, t_{1}=d^{1 / 2} t$, they write like $\dot{x}=y+x y, \dot{y}=-x+(b-d) y / d^{1 / 2}-d^{1 / 2} x y+n y^{2}$ with $d n \neq 0$ and $b^{2}-4 n d<0$. Putting $b$ instead of $(b-d) / d^{1 / 2}$ and $m$ instead of $-d^{1 / 2}$ we obtain (c).

Systems (I.e.1), (I.e.2) and (I.e.3) with a point of index 1 at origin and in coordinates $x_{1}=x, y_{1}=y /(-a)^{1 / 2}, t_{1}=(-a)^{1 / 2} t$, write like $\dot{x}=y-$ $-(-a)^{1 / 2} x^{2}+x y, \dot{y}=-x+b y+\ell x^{2}+m x y$, where either $(a+b+\ell)^{2}-4 a(\ell+m)<$ 0 , or $b=m, a=\ell+m<0$. Taking the new coordinates $x_{1}=x, y_{1}=-y$, $t_{1}=-t$ and putting $p, b, \ell+b p$, and $m+b$ instead of $(-a)^{1 / 2},-b, \ell$, and $-m$, respectively; we obtain case (e) for the first condition and case (d) for the second one.

From $[\mathrm{L}]$ or Appendix $\mathrm{B}$ of $[\mathrm{CGL}]$ we can give Table 5.1 that provides the 
focal quantities $W_{1}, W_{2}$ and $W_{3}$ for systems of Theorem 5.1.

\begin{tabular}{c|c|c|c|c|c}
\hline Syslen & $(\mathrm{l})$ & $(\mathrm{b})$ & $(\mathrm{c})$ & $(\mathrm{d})$ & $(\mathrm{e})$ \\
\hline$W_{1}$ & $-n>0$ & $-\ell(2+m) \geq 0$ & $-m n>0$ & $3 p>0$ & $p(1-2 \ell)-m \ell$ \\
\hline$W_{2}$ if $W_{1}=0$ & - & 0 & - & - & $\ell p(2 p+m)(3 p-m)(p m-\ell)$ \\
\hline$W_{3}$ if $W_{1}=W_{2}=0$ & - & 0 & - & - & $10 p^{3} \ell^{2}\left(3 p^{2}-\ell\right)>0$ \\
\hline
\end{tabular}

Table 5.1. Focal quantities for the $Q S 1$ of Theorem 5.1 .

Proposition 5.2. (i) Systems (a), (b), (d) and (e) of Theorem 5.1 are semicomplete families of rotated vector fields (SFR) with parameter b, (mod. $y=0),\left(\bmod . y+x^{2}=0\right),\left(\bmod \cdot y+p x^{2}+x y=0\right)$ and $\left(\bmod \cdot y+p x^{2}+x y=0\right)$, respectively (see for definitions [Du] and [Pl] or Appendix $D$ of [CGL]).

(ii) System (c) of Theorem 5.1 is SFR with parameter b, (mod. $y=0)$ in the half plane $1+x>0$, and $x=-1$ is an invariant straight line for the flow of (c).

The proposition follows easily by direct computations.

Theorem 5.3. (see the proof in the Appendix). Systems (a), (b), (c) and (d) of Theorem 5.1 have at most one limt cycle.

System (e) of Theorem 5.1 under condition $(m+b-p)^{2}-4(\ell+b p) \leq 0$ has at most one limit cycle.

Corollary 5.4. Systems (a), (b), (c) and (d) of Theorem 5.1 have no limit cycles when $b \leq 0$ or $b \geq b^{*}$, where $b^{*}$ is a positive function of the other coefficients of the above systems. Furthermore $b^{*}<2$.

Proof: From Table 5.1 it follows that $W_{1}>0$ for systems (a), (b), (c) and (d). Then, we know that for our systems, a stable limit cycle rises from the origin when $b \geq 0$. By Proposition 5.2 our systems are SFR with parameter $b$. Hence, we can apply Theorem Dl of Appendix D of [CGL]. Furthermore, in these cases, by Theorem 5.3, we have that there exists at most one limit cycle for all values of $b$. Then we know that the limit cycle that appears from the origin when $b \geq 0$ grows with $b$. Since $(0,0)$ is the unique singularity of our systems, these limit cycles must disappear in an infinite separatrix cycle for some value of $b$, called $b^{*}$. Of course $b^{*}<2$ by Proposition 1.9 . Hence, by the property of non intersection of the limit cycles of SFR the corollary follows. Note that we have not taken into account system (B) with $W_{1}=0$, since for this system we know, by Table 5.1 , that $W_{2}=W_{3}=0$ and hence the origin is a topological center when $b=0$. By consequence it has no limit cycles when $b \neq 0$.

Now, we shall give the phase portraits of systems of Theorem 5.1 case by case (without taking into account if some phase portrait has been already appeared in previous sections). 
System (a). For this system the behaviour in a neighbourhood of infinity is always the same. Note also that the straight line $y=1$ is invariant if $b+n=0$ and it is without contact if $b+n \neq 0$. Now we show that if $b+n \geq 0$ system (a) has no limit cycles. First, we consider the vector field (a) multiplied by the factor $1 /(1-y)$ in the half plane $y<1$ in which limit cycle can exist. Then we have

$$
\frac{\partial}{\partial x}(y /(1-y))+\frac{\partial}{\partial y}\left(-x+\left(b y+n y^{2}\right) /(1-y)\right)=\left(b+2 n y-n y^{2}\right) /(1-y)^{2} \geq 0
$$

and by Bendixson criterion (see [L, pp. 238]), we have that system (a) has no limit cycles.

In [R] there is a numerical determination of the curve $b^{*}(n)$ for which the limit cycle disappears. Our results plus these numerical results are showed in Figure 5.1. In short, the phase portraits of system (a) are drawn in Figure 5.2.

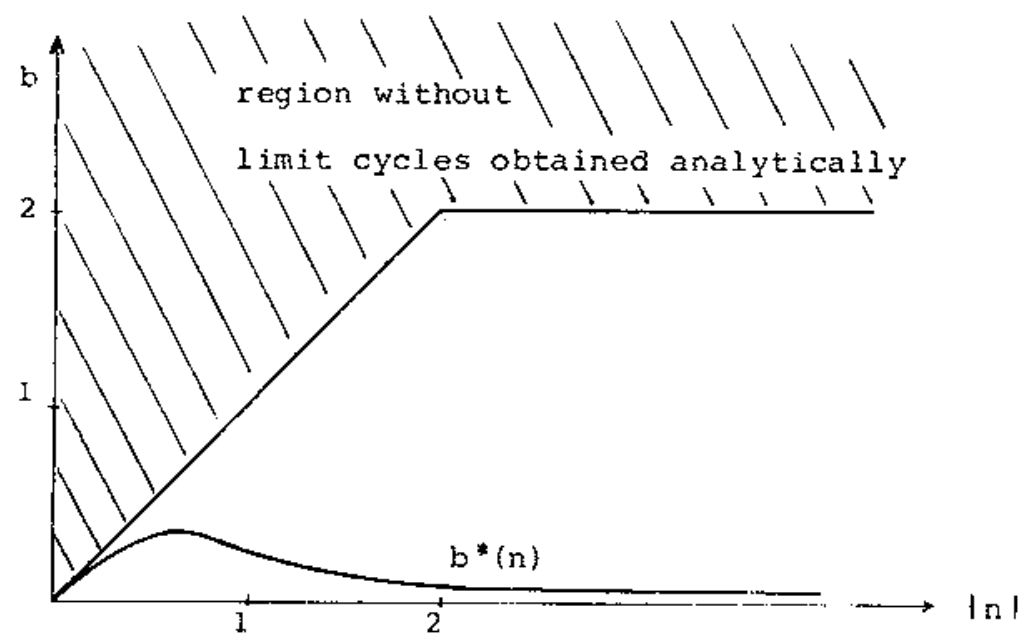

Figure 5.I. Numerical results obtained in $[\mathbf{R}]$ that show the values $b^{*}(n)$ for which the limit cycle of system (a) disappears. 


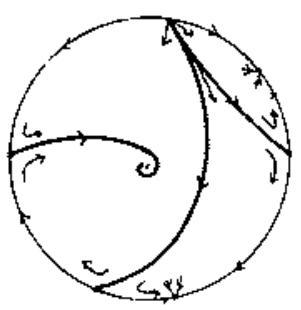

$b \leqslant 0$

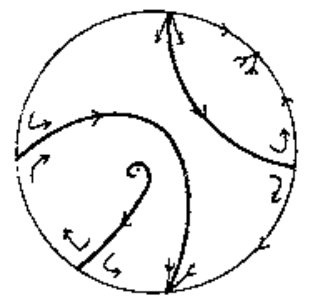

$\mathrm{b}^{*}<\mathrm{b}<-\mathrm{n}$

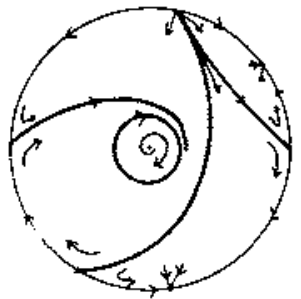

$0<h<b^{*}$

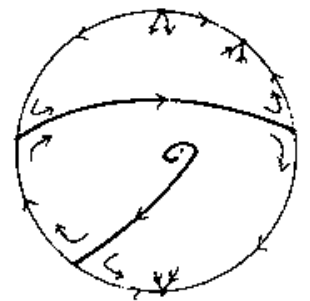

$b=-n$

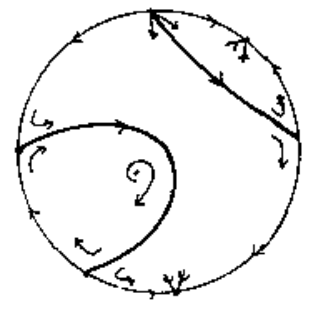

$b=b^{*}$

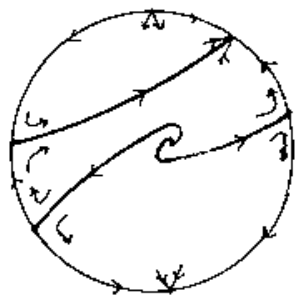

$\mathrm{b}>-\mathrm{n}$

Figure 5.2. Phase portraits of system (a) of Theorem 5.1.

System (b). From Figure 3.2 we have that the behaviour at infinity of system (b) can be of four different forms according with $m \geq 2,1<m<2$, $m=1$ or $0<m<1$.

The following lemma has a similar proof to the proof of Lemma 20 of [CGL].

Lemma 5.5. System (b) with $|b|<2$ satisfies the following properties:

(i) The straight line $y=b x+1 / m$ is invariant if brn $+\ell=0$ and without contact if $b m+\ell \neq 0$.

(ii) If $m \neq 2,4$ the parabola $y=(m-2) / 2 x^{2}-Q x-\left(Q^{2}-b Q-1\right)$ where $Q=(b m+2 \ell) /(m-4)$ is a trajectory of system $(b)$ if $Q=b$. If $Q \neq b$ it is without contact.

(iii) The parabola given in (ii) when $Q=b$ is a separatrix of the singularity $(0,0)$ of the local chart $U_{2}$.

In short, by Lemma 5.5, Theorem 5.3, Corollary 5.4, the behaviour at infinity and the properties of the families of rotated vector ficlds we obtain Figures 5.3,5.4, 5.5, and 5.6 (in all these figures it is assumed that $\ell<0$ to clarify the evolution). The figure 5.7 can be obtained integrating the system (b). 


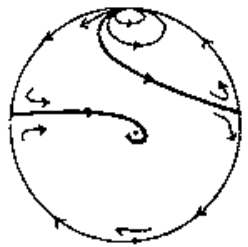

$b \leqslant 0$

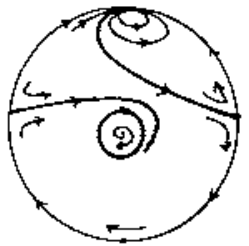

$0<\mathrm{b}<-2 / \mathrm{m}$

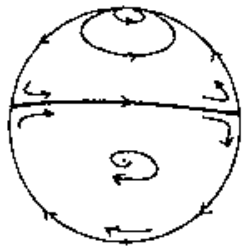

$b=-\ell / n$

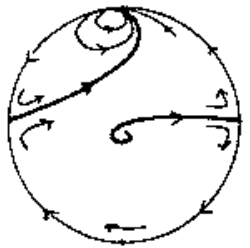

$b>-\ell / m$

Figure 5.3. All the possible phase portraits of system (b) of Theorem 5.1 when $m \geq 2$ and the origin is not a topological center.

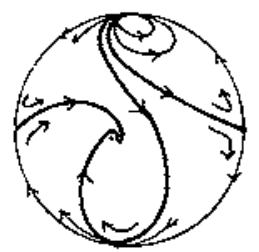

$b \leqslant 0$

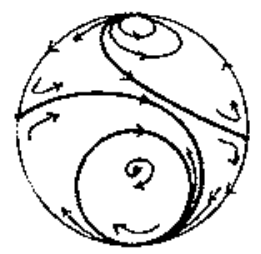

$b=-\ell / 2$

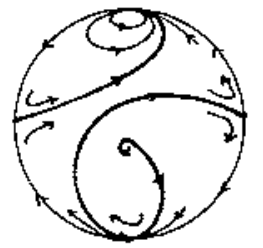

$\mathrm{b}=\mathrm{b}_{2}^{*}$

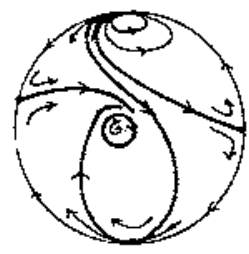

$0<\mathrm{b}<\mathrm{b}$

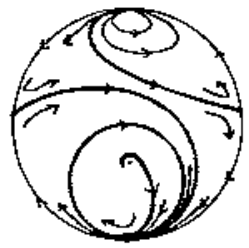

$-\ell / 2<b<-\ell / m$

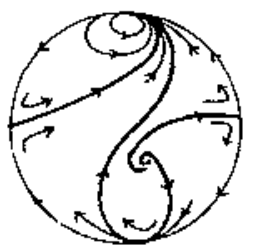

$b>b_{2}^{*}$

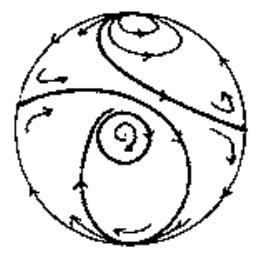

$\mathrm{b}=\mathrm{b}_{1}^{*}$

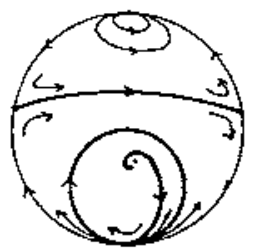

$b=-2 / m$

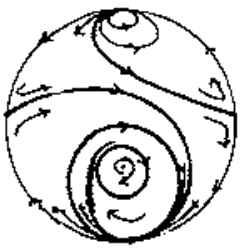

$b_{i}<b<-\ell / 2$

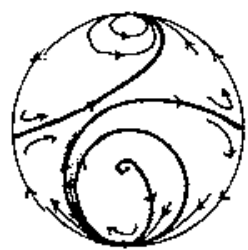

$-\ell / a<b<b_{2}^{*}$

Figure 5.4. All the possible phase portraits of system (b) of Theorem 5.1 when $1<m<2$ and the origin is not a topological center. 


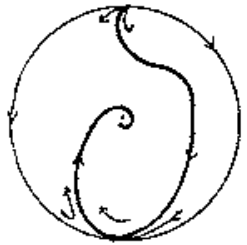

$b<0$

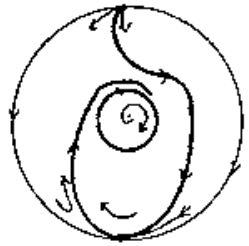

$0<b<-\ell / 2$

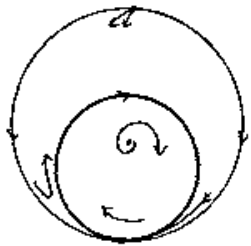

$b=-\ell / 2$

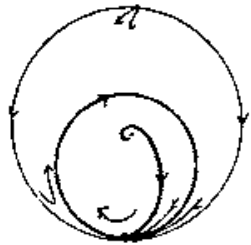

$b>-\ell / 2$

Figure 5.5. All the possible phase portraits of system (b) of Theorem 5.1 when $m=1$.

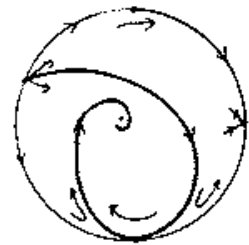

$b \leqslant 0$

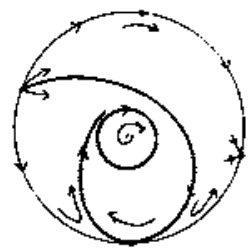

$0<b<-\ell / 2$

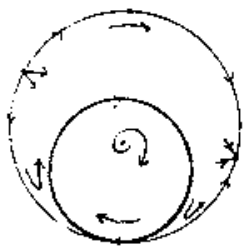

$\mathrm{b}=-\ell / 2$

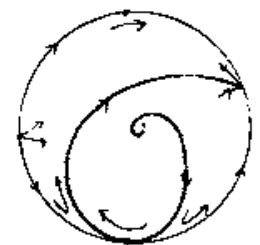

$b>-2 / 2$

Figure 5.6. All the possible phase portraits of system (b) of Theorem 5.1 when $0<m<1$ and the origin is not a topological center.
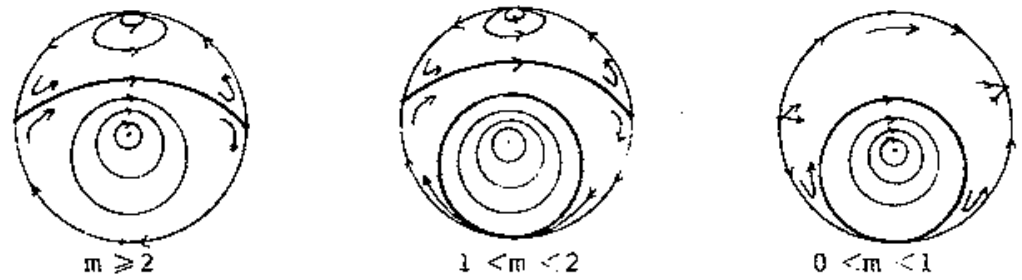

Figure 5.7. Phase portraits of system (b) of Theorem 5.1 with $\ell=b=0$. 
System (c). This sytem has the straight line $x=-1$ invariant. By Theorems $E$ and $S$ we can study the behaviour at infinity and we obtain three different cases according with $0<n<1, n=1$ and $n>1$. By using the same techniques that in the above case we obtain Figures 5.8,5.9 and 5.10.

Systems (d) and (e). First we study the behaviour at infinity of these systems and we obtain the different possibilities showed in Figure 5.11. These possibilities depend on the sign of $A=m+b+p$ and on the number of infinite singularities. Note that if systems (d) and (e) have two or three infinite singularities then $A \neq 0$.

We can prove that if $A<0$ then $b \leq-2$ and hence the origin is a stable node. This fact implies that in this case the phase portraits of systems (d) and (e) with two or three infinite singularities are one of the pictures of Figure 5.12.

For system (e) we can give a result similar to Corollary 5.4.

Proposition 5.6. System (e) under conditions $(m+b-p)^{2}+4(\ell+b p) \leq 0$ and with $b \leq 0$ has no limit cycles.

Proof: From Theorem 5.3 we know that system (e) under the condition $(m+b-p)^{2}+4(\ell+b p) \leq 0$ has at most one limit cycle. Furthermore by looking at Theorem A5 of the Appendix we can affirm that it can exist only when $b \neq 0$ and it is hyperbolic. Note also that system (e) has one (resp. two) singularities at infinity if $(m+b-p)^{2}+4(\ell+b p)<0$ (resp. $\left.=0\right)$. So, if system (e) has one infinite singularity, by Figure 5.11 and the hyperbolicity of the limit cycle the result follows studying the stability of the origin. Now, we consider the case in which (e) has two infinite singularities. This case is the boundary of the above one. Note that if the limit cycle would exist, by Theorem A5 it would be hyperbolic and hence it would persist by changing $\ell$ by $\ell+\epsilon$, with $\epsilon<0$, in system (e). In short, it does not exist.

By Proposition 5.6 and the above results we obtain that when systems (d) and (e) have a unique infinite singularity their phase portrait are the ones showed in Figure 5.13.

The phase poitrait of systcm (d) will be now determinated by using these last results, the properties of the $S F R$, and the results Section 7 of [CGL] (after taking a suitable change of coordinates). Note that the number of infinite singularities is three, two or one according with $(b+p)^{2}-4>0,=0$ or $<0$, respectively. The phase portraits of system (d) are showed in Figure 5.14. 
QS WITH ONE REST POINT

249
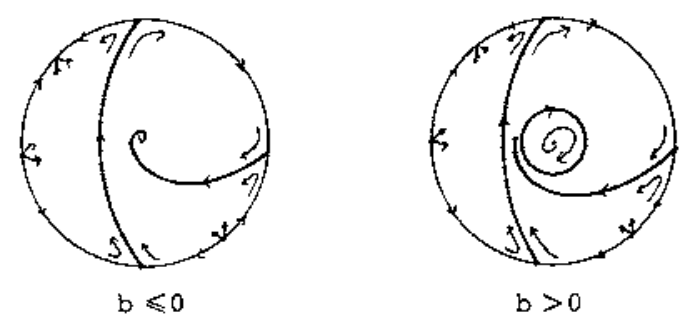

Figure 5.8. Phase portraits of system (c) of Theorem 5.1 when $0<n<1$.
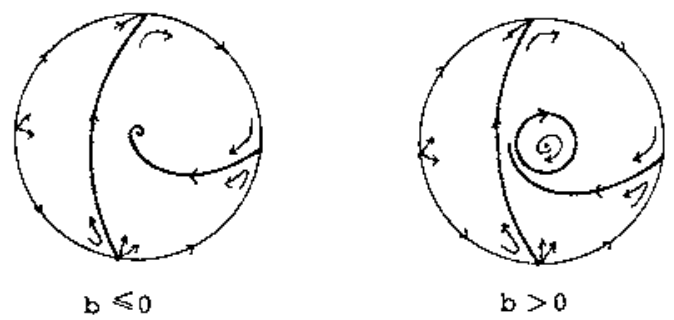

Figure 5.9. Phase portraits of system (c) of Theorem 5.1 when $n=1$.
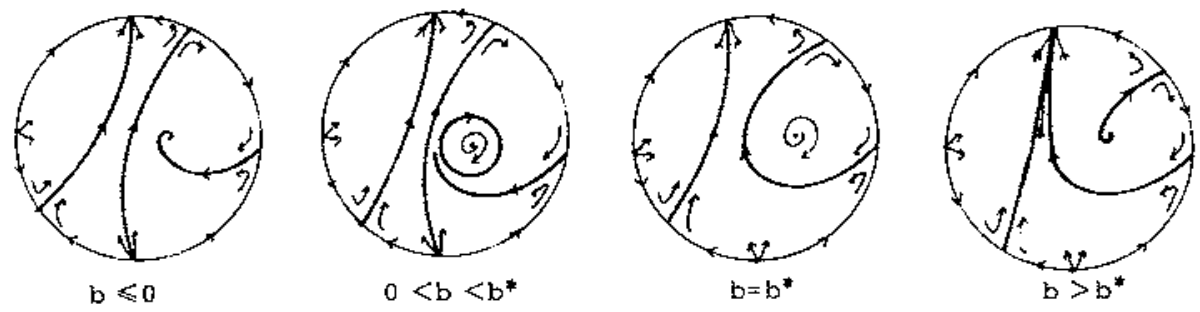

Figure 5.10. Phase portraits of system $(c)$ of Theorem 5.1 when $n>1$. 


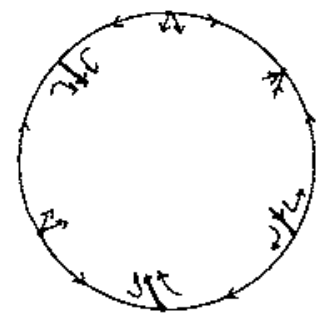

$A>0$

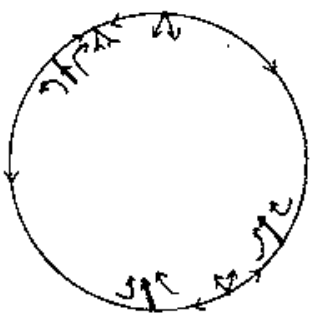

A $<0$

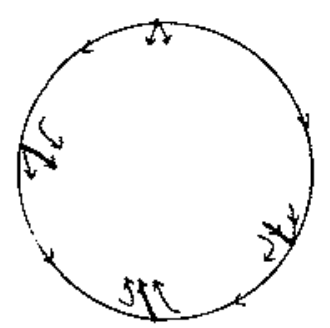

$A>0$

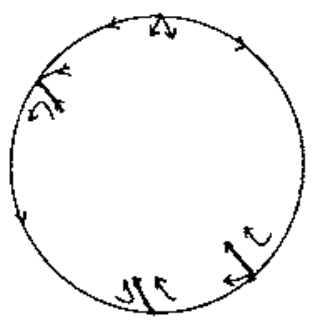

$\mathrm{A}<0$

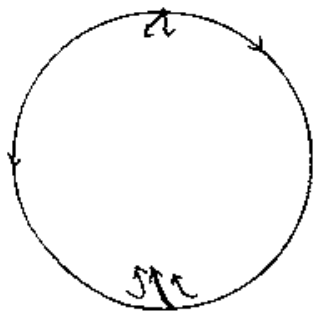

Figure 5.11. Phase portraits at infinity for systems $(d)$ and $(e)$ of Theorem 5.1, where $A=m+b+p$.
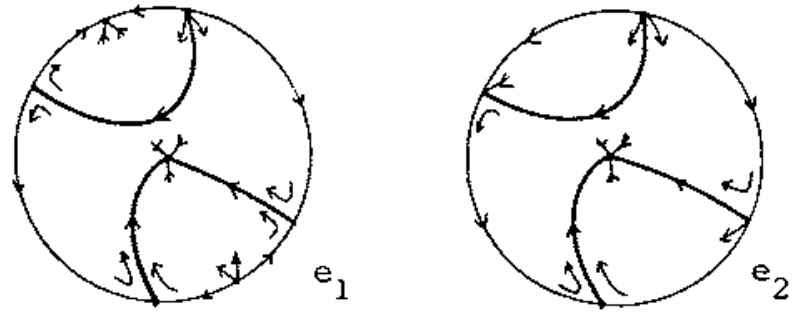

Figure 5.12. Phase portraits of systems $(d)$ and $(e)$ of Theorem 5.1 with two or three infinite singliarities and $m+b+p<0$. 


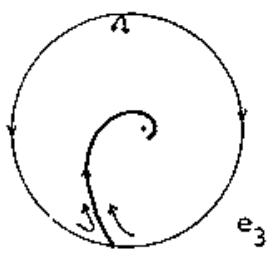

$b \leqslant 0$

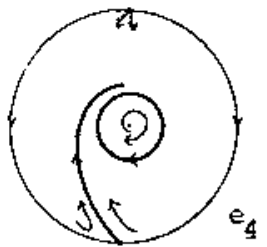

$b>0$

Figure 5.13. Phase portraits for systems $(d)$ and $(e)$ of Theorem 5.1 and a unique infinite critical point.

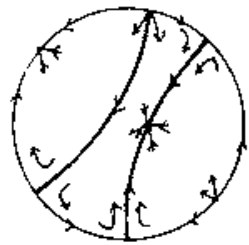

$b<-2-p$

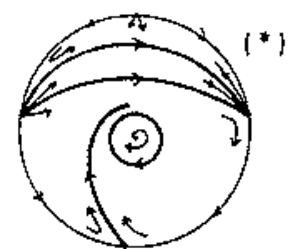

$b=2-p$

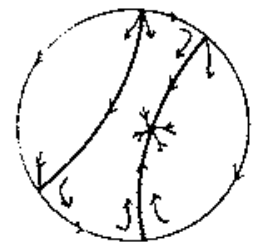

$b=-2-p$

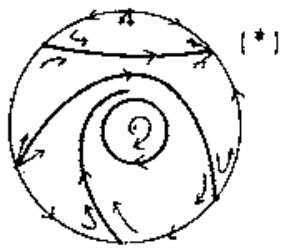

$2-p<b<b^{*}$

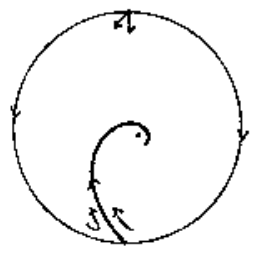

$-2-p<b \leqslant 0$

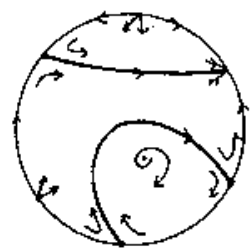

$b=b^{*}$

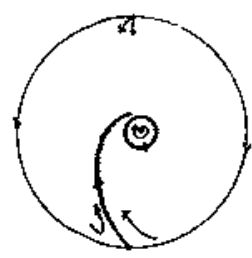

$0<b<2-p$

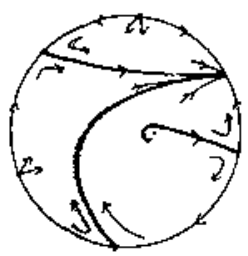

$\mathrm{b}>\mathrm{b}^{*}$

Figure 5.14. Phase portrait of system (d) of Theorem 5.1 (in this figure it is assumed that $0<p<2$, in general the limit cycle can appear in pictures with (*)). 
The remainder case (e) is the most complicated one. By results of $[B]$, if we take a system (e) with the conditions $W_{1}=W_{2}=0$ and $W_{3}>0$ we can modify its coefficients in order to obtain a $Q S 1$ of type (e) with at least three limit cycles surrounding the $(0,0)$. Examples of $Q S$ for which three limit cycles aipear from a weak focus are given in several papers, see for instance [Che], [P2], [Sh]. In order to give a dynamic interpertation of the more complete evolution of the phase portraits of this system we will fix the values of $p$ and $m$ such that $-1+2(p m)^{1 / 2}>0$ and $m-p<0$ (the other cases are similar and they give no new phase portraits). Hence we have that $-1-2(\mathrm{pm})^{1 / 2}<\ell<-1+2(\mathrm{pm})^{1 / 2}$.

The number of infinite singularities is three, two or one according with $\ell>B=\left(-b^{2}-2(p+m) b-(m-p)^{2}\right) / 4, \ell=B$ or $\ell<B$, respectively. Furthermore, if $\ell=-b m$ the straight line $y=b x+1 / m$ is invariant by the flow of system (e).

In Figure 5.15 we represent the plane of parameters $b, \ell$, the conditions of number of singularities at infinity, and the existence of an invariant straight line when $\ell=-b m$. There are some regions for which the phase portraits are already determined using the above considerations. These results are summarized in Table 5.2. In this Table there are some new phase portraits that have been obtained by studying the vector field (e) on the straight lincs $y=b x+1 / m$, and $y=y_{1} x+\left(y_{1}^{2}-b y_{1}+1\right) /\left(m+b-y_{1}\right)$, where $y_{1}$ is the slope of the direction associated to the lower singularity at infinity in the local chart $U_{1}$. Also, we have taken into account that system (e) under condition $\ell=-b m$ can be written like system (c), transporting the invariant straight line to $x=-1$. All its possible phase portraits are showed in Figure 5.16.

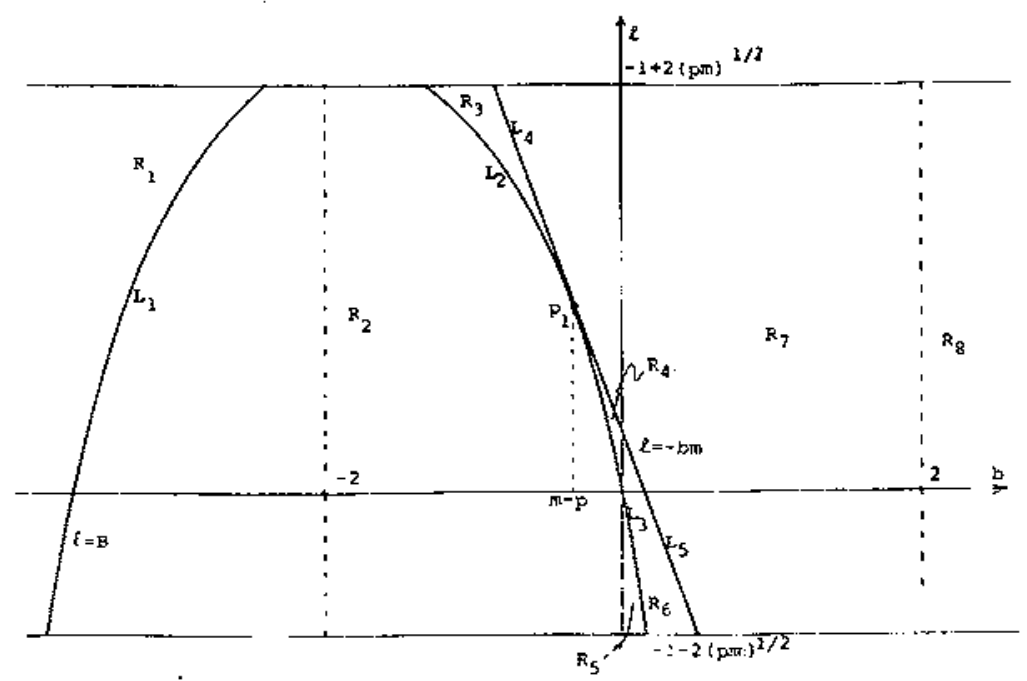

Figure 5.15. Plane of parameters $b$ and $\ell$ for the system (e) of Theorem 5.1 when $m$ and $p$ are fixed and such that $-1-2(p m)^{1 / 2}<\ell<-1+2(p m)^{1 / 2}$, and $m-p<0$. Here $B=\left(b^{2}-2(p+m) b-(m-p)^{2}\right) / 4$. 

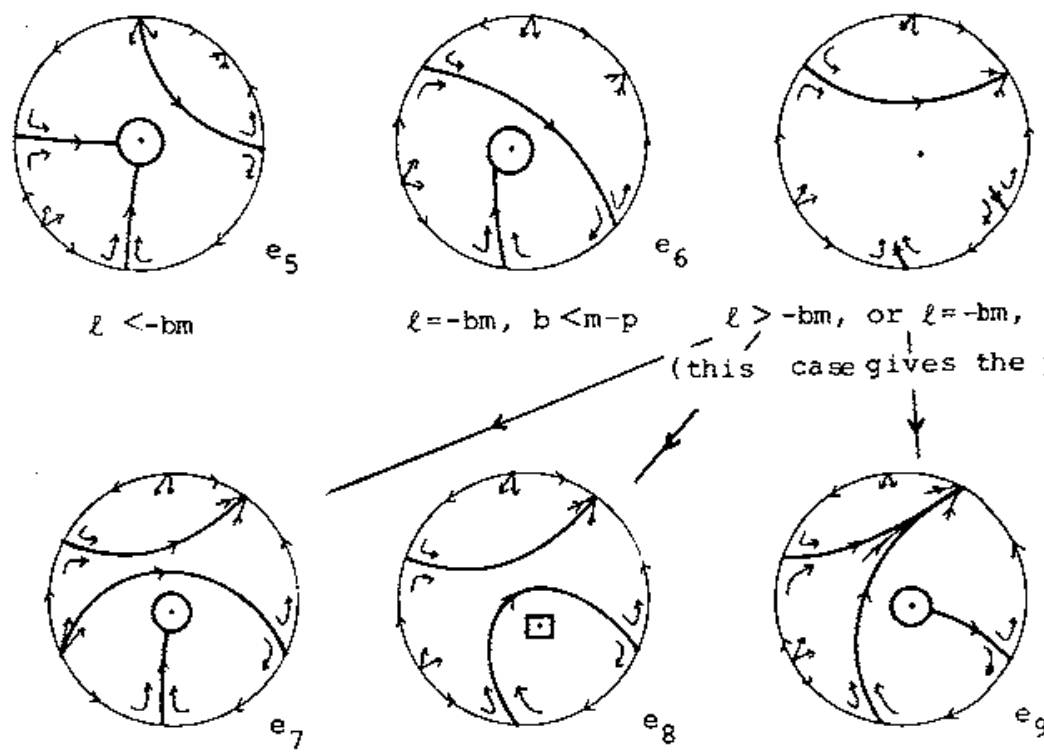

$\ell>-b m$, or $\ell=-b m, b>m-p$
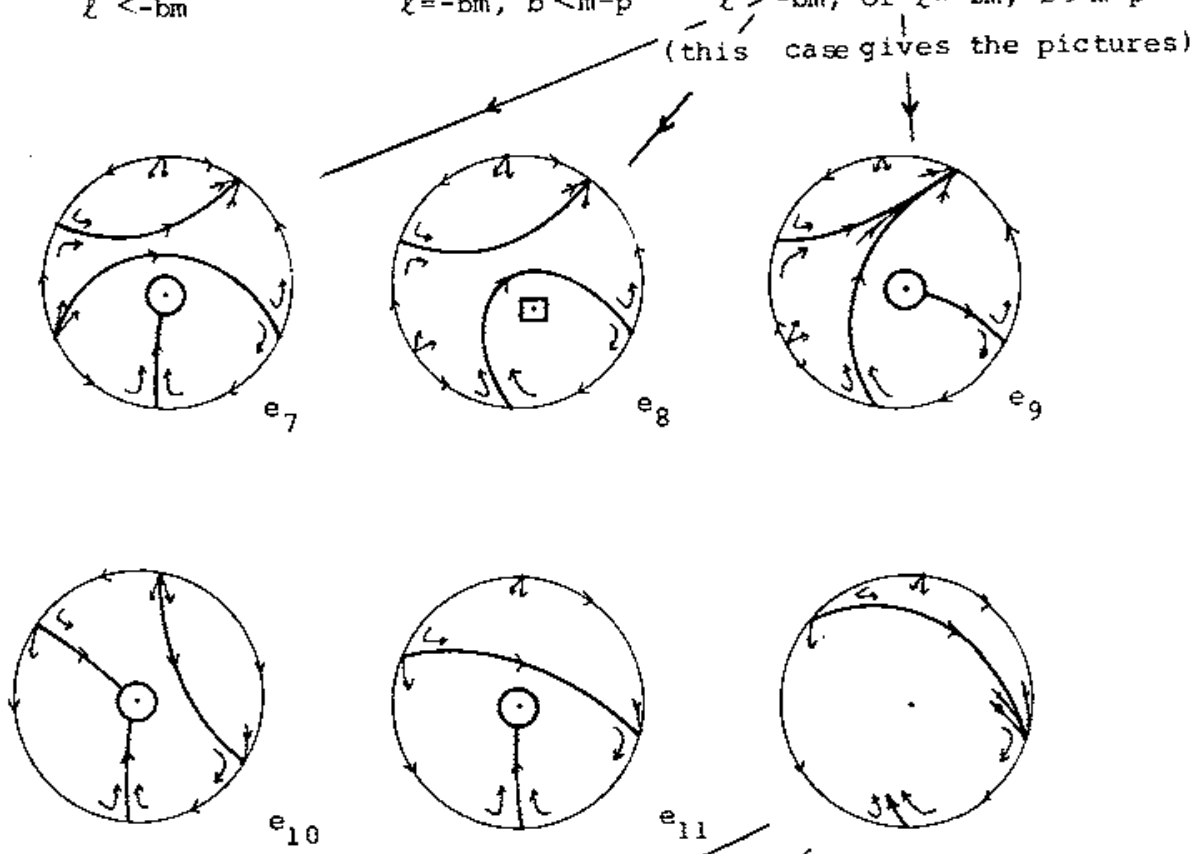

$\ell<-b m$

$e_{10}$

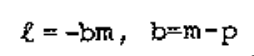

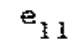

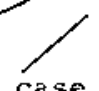

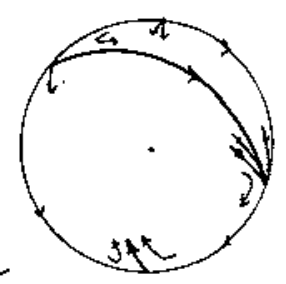

$\ell>-b m$

(this case gives the pictures)
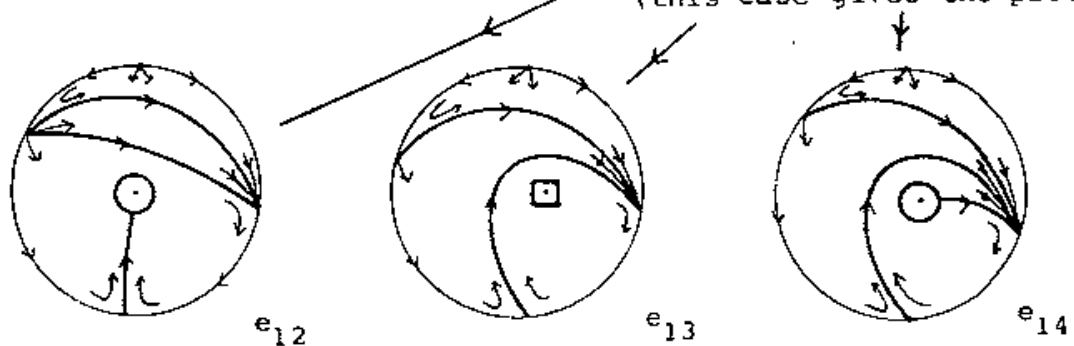

Figure 5.16. Possible phase portraits for system (e) different from $e_{1}, e_{2}, e_{3}$ and $e_{4}$ of Figures 5.12 and 5.13. The simbol $\rightarrow \odot$ is defined in Figure 3.1. The symbol $\checkmark$ denotes that we do not know the stability of the grafic. In picture $e_{8}$ we do not know either the number of linit cycles. 
Note that, at this moment, the realization of most of cases of Figures 4.4 and 4.10 have been already done in the study of systems (a), (b), (c), (d) and (e). The results obtained are in Tables 5.3 and 5.4.

\begin{tabular}{c|c}
\hline Phase portrail & Region \\
\hline$e_{1}$ & $R_{2}$ \\
\hline$e_{2}$ & $L_{1}$ \\
\hline$e_{3}$ & $R_{2}$ \\
\hline$e_{4}$ & $R_{5}$ \\
\hline$e_{5}$ & $R_{3}$ \\
\hline$e_{6}$ & $L_{4}$ \\
\hline$e_{7}$ & $L_{5}$ \\
\hline
\end{tabular}

\begin{tabular}{c|c}
\hline Phare portrsit & Region \\
\hline$e_{8}$ & $L_{5}, R_{8}$ \\
\hline$e_{9}$ & $L_{5}, R_{5}$ \\
\hline$e_{10}$ & $L_{2}$ \\
\hline$e_{13}$ & $P_{1}$ \\
\hline$e_{12}$ & $L_{3}$ \\
\hline$e_{13}$ & $?$ \\
\hline$e_{14}$ & $?$ \\
\hline
\end{tabular}

Table 5.2. Realization of some phase portraits of Figures 5.12,5.13 and 5.16 by system (e) of Theorem 5.1. The regions $R_{i}, L_{i}$, and $P_{i}$ are given in Figure 5.15 .

\begin{tabular}{c|c}
\hline System of Theorem 5.1 & has the phase portrait \\
\hline (a) with $b>-n$ & $E_{31}$ \\
\hline (a) with $b=-n$ & $E_{33}$ \\
\hline (a) with $b^{*}<b<-n$ & $E_{33}$ \\
\hline (a) with $b=b^{*}$ & $E_{34}$ \\
\hline (c) with $b=b^{*}, n>1$ & $E_{35}$ \\
\hline (a) with $b \leq 0$ & $E_{36}$ \\
\hline
\end{tabular}

Table 5.3. Realization of the phase portraits of Figure 4.4.

\begin{tabular}{c|c}
\hline System of Theorem 5,1 & has the phase portrait \\
\hline (d) with $b=-2-p$ & $E_{50}$ \\
\hline (c) with $b \leq 0, n=1$ & $E_{51}$ \\
\hline (c) with $b=2-p$ & $E_{52}$ \\
\hline$?$ & $E_{53}, E_{54}, E_{55}$ \\
\hline
\end{tabular}

Table 5.4. Realization of some phase portraits of Figure 4.10. 
In short, from all the results that we have obtained since now, the problems that we do not have solved yet, in order to find all the possible phase portraits for $Q S 1$, taking into account its number of limit cycles, are the following:

(P1) Determine the maximum number of limit cycles that can have phase portraits $e_{5}, e_{7}, e_{8}$ and $e_{9}$ of Figure 5.16.

(P2) Determine if there is some QS1 with phase portraits $e_{13}$ and $e_{14}$ of Figure 5.16 (Note that this problem is equivalent to the problem of determining $Q S 1$ with phase portraits $E_{53}, E_{54}$ and $E_{55}$ of Figure 4.10 ).

We do not solve problem (P1). We know that there are some $Q S$ with these phase portraits with at least three limit cycles, but we have not found a bound for the number of limit cycles for system (e) of Theorem 5.1 with condition $(m+b-p)^{2}+4(\ell+b p)>0$.

In Figure 5.17 we represent all the information about limit cycles that we have for system (e). The zone dotted represents the zone for which a limit cycle appears from the origin. This information will be useful in order to give a partial answer to problem (P2).

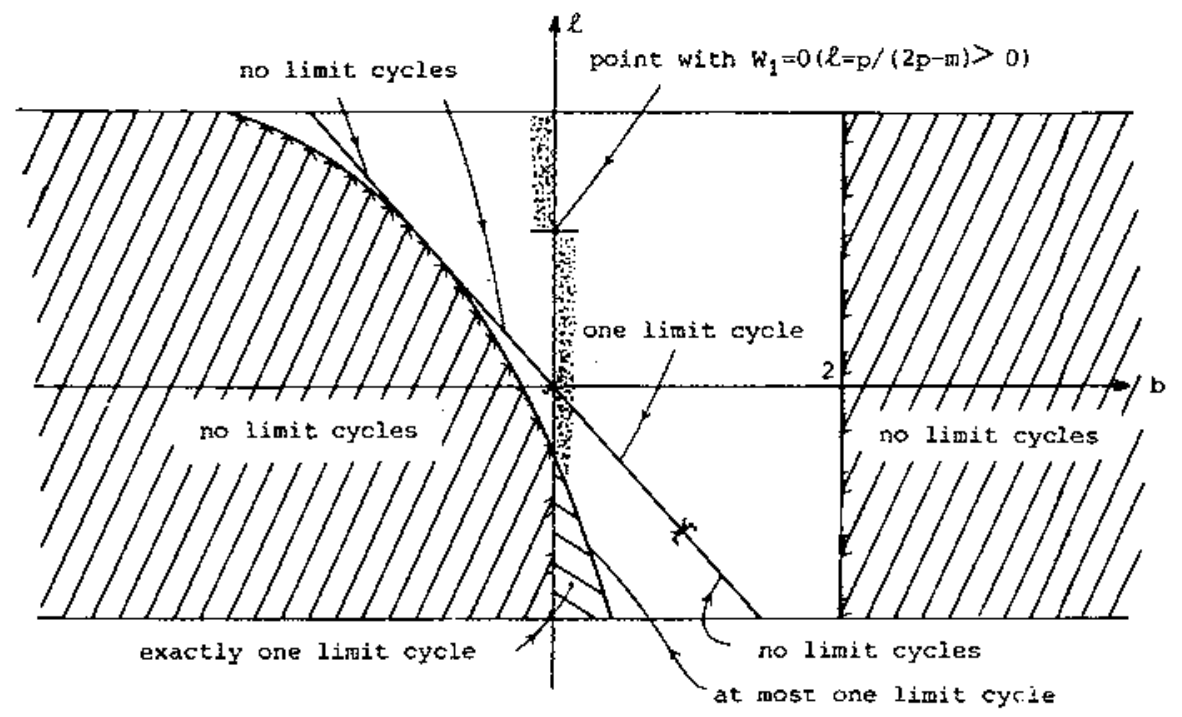

Figure 5.17. Knowlege about limit cycles for system (e) of Theorem 5.1 in the space of parameters $\ell, b$. 
Lemma 5.7. Phase portrait $e_{14}$ of Figure 5.16 is not realizable for QS1. Furthermore if picture $e_{13}$ of Figure 5.16 is realizable, it is the phase portrait of some system of type (e) of Theorem 5.1 with condition $(m+b-p)^{2}+4(\ell+b p)=0$.

Proof: Note that the two infinite critical points of a $Q S 1$ that has phase portraits $e_{13}$ or $e_{14}$ must be of type $S$. There is a few number of cases such that they have these kind of points at infinity: (I.e.6), (I.s.9), (II.e.5), (II.e.9), (II.s.8) and (II.s.13). The cases (II.e.5), (II.s.13) and (II.s.8) with condition $(E)$ have an invariant straight line through the origin. The finite critical point of systems (II.s.8) with condition $(D)$ and (I.s.9) with $\ell+b \neq 0$ is a saddlenode. The phase portrait of system (I.s.9) with condition $(D)$ and $\ell+b=0$ can not be $e_{13}$ or $e_{14}$ (look at the vector field on the straight line $y=0$ which is without contact and study the infinite singularities). The case (II.e.9) has been already studied. So the unique case that can have phase portraits $e_{13}$ or $e_{14}$ is $(Y, e .6)$. This case is equivalent to system (e) of Theorem 5.1 with condition $(m+b-p)^{2}+4(\ell+b p)=0$ and to system (d) of the same theorem with condition $(b-p)^{2}-4=0$. The second one has been already studied.

So, in order to prove that the phase portrait $e_{14}$ is not realizable for $Q S 1$, we only must consider case (e) with condition $(m+b-p)^{2}+4(\ell+b p)=0$. By Proposition 5.6 we know that this phase portrait is not realizable for $b \leq 0$. When $b>0$, we can prove also that phase portrait $e_{14}$ is not realizable. In this way consider Figure 5.17 and use the same argument that in Section 7 of [CGL]. Note that we can not utilize the same argument in order to prove that phase portrait $e_{13}$ is not realizable because in this case, system $(e)$ is not a SFR on the parabola $(m+b-p)^{2}+4(\ell+b p)=0$.

\section{Appendix. Theorems on uniqueness of limit cycles for $Q S$.}

In this appendix we enunciate some results on uniqueness of limit cycles for $Q S$. By using these results we will prove Theorem 5.3 .

Theorem A1. (see [Y1], [Y2, pp. 269]). Consider the system

$$
\dot{x}=-y+b x+\ell x^{2}+m x y+n y^{2}, \quad \dot{y}=x .
$$

Then the following hold.

(I) If $b=0, m(\ell+n)=0$, then $(E .1)$ has a center at $(0,0)$. If $b=0$, $m(\ell+n) \neq 0$, then $(E .1)$ has no periodic orbits.

(II) If $b m(\ell+n)>0$ then $(E .1)$ has no periodic orbits.

(III) If $b m(\ell+n)<0$ and $0<|b|<b^{*}=f(\ell, m, n)$ where $f$ is a function of $\ell, m, n$ then (E.1) has one and only one limit cycle, which grows as $|b|$ increases and becomes a singular cicle (homoclinic orbit) when $|b|=b^{*}$. If $|b|>b^{*}$, then (E.1) has no periodic orbits.

Theorem A2. (see [Ch]). The system

$$
\dot{x}=-y+d x+\ell x^{2}, \quad \dot{y}=x(1+a x+b y),
$$


has at most one limit cycle surrounding the origin.

Theorem A3. (see [CZ, Theorem 2]). Given the system

$$
\dot{x}=y(x+1), \quad \dot{y}=-x+c y+a x^{2}+b x y+d y^{2}
$$

the following hold.

(i) If $a \geq 0, c>0, d>0$ or $-1 \leq a \leq 0, c>0, d>0, a+d>0$, then it has at most one limit cycle.

(ii) If $b c(a+d) \geq 0$, it has no limit cycles on the half plane $x>-1$.

Theorem A4. (see [Z, Theorem 5]). The system

$$
\dot{x}=y+y^{2}, \quad \dot{y}=-x+a y+b x y+c y^{2},
$$

has at most one limit cycle if $b<0$.

Theorem A5. (see Theorem A of [CGL]). If the function $F(x, y) g(x, y)=\left[(x-b y) P_{2}(x, y)+y Q_{2}(x, y)\right]\left[x Q_{2}(x, y)-y P_{2}(x, y)\right]$ associated to the differential equations

$$
\dot{x}=y+P_{2}(x, y), \quad \dot{y}=-x+b y+Q_{2}(x, y),
$$

does not change of sing then this system has at most one limit cycle that surrounds the origin, and it is hyperbolic. Furthermore, when $b=0$ it has no limit cycles.

Proof of Theorem 5.3: System (a) writes in the form (E.1) with $\ell=n$, $m=1$ and $n=0$ after interchanging $x$ and $y$. So by Theorem A1, system (a) has no limit cycles when either $b \leq 0$ or $b>b^{*}$, and at most one limit cycle when $0<b<b^{*}$ for some $0<b^{*}=f(n)<2$.

System (b) in coordinates $x_{1}=x, y_{1}=b x-y$ writes like $\dot{x}=-y+$ $+b x+x^{2}, \dot{y}=x(1+m y-(\ell+b m) x)$ and hence, from Theorem A2, it has at most one limit cycle.

System (c) is already in the expression (E.3) with $c=b, a=0, b=m$ and $d=n$. So, system (c) has at most one limit cycle when $b>0$ and has no limit cycles when $b \leq 0$ (note that $x=-1$ is an invariant straight line for our system and the critical point is in the half plane $x>-1$ ).

System (d) in the new system of coordinates $x_{1}=b x-y, y_{1}=x$ writes in the form $\dot{x}=y+y^{2}, \dot{y}=-x+b y-x y+(p+b) y^{2}$. So, since the coefficient of $x y$ is -1 , by Theorem A4 system (d) has at most one limit cycle.

The expression of system (e) is like (E.5). So $P_{2}(x, y)=p x^{2}+x y$, $Q_{2}(x, y)=(\ell+b p) x^{2}+(m+b) x y$, and hence $F(x, y) g(x, y)=x^{2}\left(m y^{2}+(\ell+\right.$ $\left.+1) x y+p x^{2}\right)\left((\ell+b p) x^{2}+(m+b-p) x y-p y^{2}\right) \leq 0$ when $(m+b-p)^{2}+4(\ell+b p) \leq 0$. Therefore we can apply Theorem $A 5$, and system (e) with the above condition has at most one limit cycle.

New proofs of some of these theorems are recently given by Coppel, see $[\mathrm{C} 2]$ and $[\mathrm{C} 3]$. 


\section{References}

[A] J. ARGEMI, Sur les points singuliers multiples de systemes dynamiques dans $\mathrm{R}^{2}$, Ann. Mat. Pura ed Appl. ser. IV, 79 (1968), 35-70.

[ALGM] A.A. ANDRONOV, E.A. LEONTOVICH, I.I. GORDON AND A.L. MAIER, "Qualitative theory of second-order dynamic systems," John Wiley and Sons, New York, 1973.

[B] N.N. BAUTIN, On the number of limit cycles which appear with the variation of coefficients from an equilibrium point of focus or center type, Amer. Math. Soc. Transl. (1) 5 (1962), 396-413.

[C1] W.A. COPPEL, A survey of quadratic systems, J. Diff. Eq. 2 (1960), 293-304.

[C2] W.A. COPPEL, Some quadratic systems with at most one limit cycle, Research Report no. 14 (1987); preprint of the Australian National University.

[C3] W.A. COPPEL, Quadratic systems with a degenerate critical point, Research Report no 32 (1987); preprint of the Australian National University.

[Ch] L.S. Chen, Acta Mathematica Sinica, (in chinese) 20 (1977), 11-13.

[Che] LI ChENGZHI, Two problems of planar quadratic systems, Sci. Sinica (series A) XXVI (1983), 471-481.

[CI] C. CHICONE AND T. JiNGHUANG, On general properties of quadratic systems, Amer. Math. Monthly 89 (1982), 167-178.

[CZ] L.A. Cherkas AND L.I. ZHILEVICH, Some criteria for the absence of limit cycles and for existence of a single limit cycle, Diff. Equations 6 (1970), 891-897.

[CGL] B. COll, A. Gasuli AND I. Llibre, Some theorems on the existence, uniqueness and non existence of limit cycles for quadratic systems, J. of Diff. Eq. 67 (1987), 372--399.

[D] T. DATE, Classification and analysis of two-dimensional real homogeneous quadratic differential equations systems, $J$. of Diff. Eq. 32 (1979), 311-334.

[Du] G.F.D. DuFF, Limit cycles and rotated vector fields, Ann. of Math. 67 (1953), 15-31.

[DP] R.J. DICKSON AND L.M. PERKo, Bounded quadratic systems in the plane, J. of Diff. Eq. 7 (1970), 251-273.

[G] E.A.V. GONZALES, Generic properties of polynomial vector fields at infinity, Trans. of A.M.S 143 (1969), 201--222.

[GLL] A. GASUlL, SHENG LI-REN AND J. LlibRE, Chordal quadratic systems, Rocky Mountain J. of Math. 16 (1986), 751-782.

[JR] P. DE JAGER AND J.W. REYN, Phase portraits for quadratic systcms with a higher order singularity, Proceedings ICIAM 87 (1987), 85-97.

[L] S. LEFSCHETZ, "Differential Equations: Geometric theory," John Wiley and Sons; New York/London, 1963. 
[LMP] A. Lins, W. DE MELo and C.C. Puch, On Liénard's Equation, Lecture Notes in Mathematics 597 (1957), 335-357, Springer Verlag Berlin/New York.

[M] L. MARKUS, Quadratic differential equations and nonassociative algebras, Contribution to the theory of nonlinear oscillations 5 (1960), 185-213, Princeton Univ. Press.

[P1] L.M. PERKO, Rotated vector fields and the global behaviour of limit cycles for a class of quadratic systems in the plane, J. Diff. Eq. 18 (1975), $63-86$.

[P2] L.M. PERKo, Limit cycles of quadratic systems in the plane, Rocky Mountain J. of Math. 14 (1984), 619-645.

[R] Y.J. ROZET, The closure of separatrices of a first-order differential equation, Diff. Eq. 7 (1971), 1517-1521.

[Re] J.W. REYN, Phase portraits of a quadratic system of differential equations occurring frecuently in applications, Niew. Arch. Wisk. 5 (1987), 107-155.

[S] J. SOTOMAYoR, Curvas definidas por equaçoes diferenciais no plano, Instituto de Matemática Pura e Aplicada (1981), Rio de Janeiro.

[Sh] SHI Songling, A concrete example of the existence of four limit cycles for plane quadratic systems, Sci. Sinica Ser. A 23 (1980), 153-158.

[V] N.I. VULPE, Affine-invariant conditions for the topological discrimination of quadratic systems with center, Diff. Eq. 19 (1983), 273-280.

[Y1] YE YANQIAN, Some problems in the qualitative theory of ordinary differential equations, J. Diff. Eq. 46 (1982), 153-164.

[Y2] YE YANQIAN AND OTHERS, Theory of limit cycles, Trans. Math. Monographs 66; (Amer. Math. Soc., 1986).

[Z] L.I. ZHILEVICH, Separatrices and limit cycles of certain differential equations, Diff. Equations 7 (1971), 597-602.

B. Coll: Departament de Matemàtiques i Informàtica

Facultat de Ciències

Universitat de les Illes Balears

07071 Palma de Mallorca, SPAIN.

A. Gasull: Departament de Matemàtiques

Facultat de Ciències

Universitat Autònoma de Barcelona

08193 Bellaterra, Barcelona, SPAIN.

J. Llibre: Departament de Matemátiques

Facultat de Ciencies

Universitat Autónoma de Barcelona

08193 Bellaterra, Barcelona, SPAIN.

Rebut el 22 de Març de 1988 